\title{
Geometry of Multiprimary Display Colors I: Gamut and Color Control
}

\author{
Gaurav Sharma and Carlos Eduardo Rodríguez-Pardo
}

\begin{abstract}
Displays that render colors using combinations of more than three lights are referred to as multiprimary displays. For multiprimary displays, the gamut, i.e., the range of colors that can be rendered using additive combinations of an arbitrary number of light sources (primaries) with modulated intensities, is known to be a zonotope, which is a specific type of convex polytope. Under the specific three-dimensional setting relevant for color representation and the constraint of physically meaningful nonnegative primaries, we develop a complete, cohesive, and directly usable mathematical characterization of the geometry of the multiprimary gamut zonotope that immediately identifies the surface facets, edges, and vertices and provides a parallelepiped tiling of the gamut. We relate the parallelepiped tilings of the gamut, that arise naturally in our characterization, to the flexibility in color control afforded by displays with more than four primaries, a relation that is further analyzed and completed in a Part II companion paper. We demonstrate several applications of the geometric representations we develop and highlight how the paper advances theory required for multiprimary display modeling, design, and color management and provides an integrated view of past work on on these topics. Additionally, we highlight how our work on gamut representations connects with and furthers the study of three-dimensional zonotopes in geometry.
\end{abstract}

Index Terms-multiprimary displays, color gamut, gamut surface, color control, zonotope, gamut tiling.

\section{INTRODUCTION}

The CIE system [1] provides the foundation for the mathematical representation of color, which in turn serves as the basis for frameworks for a signal processing systems oriented approach to modeling and control for color devices and systems [2]-[5]. At the root of the CIE system are tristimulus, i.e. 3-tuple, representations of color that are a linear function of the spectral power distributions of light seen by the observer. For additive display systems, where the spectral power distribution produced by the display can be modeled as a linear combination of primaries, i.e., light sources whose intensities are varied, the end result is a relatively simple device model that can be represented as channel-wise nonlinearities coupled with a linear transformation [6]. For traditional three primary displays, this model can also be readily inverted and it is the basis for color management for most display devices. Compared to traditional three primary displays, multiprimary displays that use more than the three primaries, offer a key advantage that they can enlarge the gamut, i.e., the range of

G. Sharma is with the Department of Electrical and Computer Engineering, University of Rochester, Rochester, NY, 14627-0126 USA e-mail:

C.E. Rodríguez-Pardo is with the Department of Electrical and Computer Engineering, University of Rochester, Rochester, NY, 14627-0126 USA email: colors that the display can produce. The gamut is of fundamental interest in display design and color management. For traditional three primary displays, the control values required to reproduce a given color are uniquely determined, whereas this is not the case for multiprimary displays. Therefore a characterization of the flexibility available in color control and alternative strategies for color control are also of key interest for multiprimary displays.

Our work in this paper traces its roots back to geometrical representations of multiprimary display and surface color gamuts developed and introduced independently and near concurrently in [7], [8], and [9]. Specifically, for the efficient computation of gamut volumes in perceptual color spaces, we utilized parallelepiped tilings of multiprimary gamuts in [7]. Centore [8] noted that the tristimulus gamut of surface colors that are formed by (spectrally variable) reflection of incident light corresponds to (the limit of a) zonotope [10, Chap. 7], which is a specific type of convex polytope. Once the connection between the gamut of surface colors and zonotopes was established, it also became apparent that the gamut of a multiprimary display also corresponds to a zonotope and that the parallelepiped tilings in [7] correspond to tilings of the gamut zonotope. Centore and Brill [9] utilized the zonotope representation for color control in multiprimary displays. Using the zonotope representation, Centore [11] also demonstrated that color control values are unique for colors on the gamut surface. While these prior works focused on individual aspects of the mathematical representation and geometrical characterization of multiprimary display color, in this and a companion Part II paper [12], we aim to provide a complete, cohesive, relatively self-contained, and readily usable characterization of the gamut and color control for multiprimary displays. The formal notation and terminology that we adopt also allows for more precise and clear, albeit intricate, presentation than has been previously attempted. Finally, we note that our work also advances known results in geometry, specifically, in the representation of zonotopes and their tilings within the specific three-dimensional context that is relevant for color representation and under the physically meaningful constraint of nonnegative components for the vectors representing the primaries. We defer a detailed discussion of this last point to Section VI because the framework we develop also provides clearer context for the discussion.

This manuscript is organized as follows. Section II introduces notational conventions and the mathematical context for the paper. Section III introduces the physical/mathematical model for color displays that we use in our work, formally defines the gamut and color control functions, and points 
out the connections and differences with the general theory of zonotopes. Section IV develops and presents our main mathematical results as a representation theorem for the gamut of multiprimary displays and an associated algorithm for obtaining the representation. Section $\mathrm{V}$ relates theory we develop to applications, outlining how the mathematical representations we develop in Section IV can be used in the analysis and design of multiprimary displays and color control strategies. The paper concludes with a summary and discussion in Section VI. Appendices A-G provide proofs for the mathematical results, specifications of primary systems used in the examples, additional sample results, and visualizations.

\section{Notational Conventions And Preliminaries}

To streamline the subsequent presentation, this section introduces notational conventions for describing index sets, their subsets, and combinations; basic functions; topological notions of interior, exterior, and boundary; and background results from the geometry of polytopes [10], [13] that we use. For easy reference, Table I lists the symbols and notational conventions used in the paper.

TABLE I

LIST OF SYMBOLS/NOTATION

\begin{tabular}{|c|c|c|}
\hline Type & Symbol & Description \\
\hline $\begin{array}{l}\text { Scalar } \\
\text { Set }\end{array}$ & $\begin{array}{c}K \\
n, m, N, M \\
\langle M\rangle\end{array}$ & $\begin{array}{l}\text { Number of primaries } \\
\text { Generic integers } \\
\text { The set of integers }\{1,2,3, \ldots, M\}\end{array}$ \\
\hline $\begin{array}{l}\text { Indices } \\
\text { Sequence }\end{array}$ & $\underset{\mathbb{J} / \mathbb{P}}{\mathbb{I}}$ & $\begin{array}{l}\text { Generic sequence of indices } \\
\text { Sequences of two/three indices }\end{array}$ \\
\hline Set of sequences & $\begin{array}{l}\mathfrak{C}^{m}(\mathbb{I}) \\
\mathfrak{P}(\mathbb{I})\end{array}$ & $\begin{array}{l}\text { Set of all combinations of } m \text { elements from } \mathbb{I} \\
\text { Set of all permutations of the elements in } \mathbb{I}\end{array}$ \\
\hline $\begin{array}{l}\text { Vector Space } \mathbb{R} \\
M \times 1 \text { Vector } \\
\text { Set of vectors }\end{array}$ & $\begin{array}{l}\mathbf{x}, \mathbf{v}, \mathbf{w} \\
\overline{\mathcal{H}}(\mathbf{w}, v) \\
\mathcal{H}(\mathbf{w}, \tau, \nu)\end{array}$ & $\begin{array}{l}\text { Generic vectors } \\
\text { Hyperplane defined by } \mathbf{w}^{T} \mathbf{x}=v \text {, where } \mathbf{w} \text { is the } \\
\text { unit normal and } v \in \mathbb{R} \\
\text { Region of space bounded by the hyperplanes } \\
\overline{\mathcal{H}}(\mathbf{w}, \tau), \overline{\mathcal{H}}(\mathbf{w}, \nu) \text {, with } \tau<\nu\end{array}$ \\
\hline $\begin{array}{l}\text { Tristimulus spa } \\
3 \times 1 \text { Vector } \\
\text { Matrix }\end{array}$ & $\begin{array}{c}\mathbf{t} \\
\mathbf{p} \\
\mathbf{P}\end{array}$ & $\begin{array}{l}\text { General tristimulus } \\
\text { Primary tristimulus } \\
3 \times K \text { primary matrix } \\
3 \times n \text {, generic tristimulus matrices }\end{array}$ \\
\hline Set of vectors & $\begin{array}{c}\mathcal{A}, \mathcal{C} \\
\mathcal{G} \\
\mathcal{B}_{\mathcal{G}} \\
\mathcal{P}(\mathbf{v}, \mathbf{A}) \\
\mathcal{F}(\mathbf{v}, \mathbf{B}) \\
\mathcal{E}(\mathbf{v}, \mathbf{w})\end{array}$ & $\begin{array}{l}\text { Generic sets of tristimuli } \\
\text { Display gamut } \\
\text { Boundary of } \mathcal{G} \\
\text { Parallelepiped at origin } \mathbf{v} \text { spanned by matrix } \mathbf{A}_{3 \times 3} \\
\text { Parallelogram at origin } \mathbf{v} \text { spanned by matrix } \mathbf{B}_{3 \times 2} \\
\text { Line segment at origin } \mathbf{v} \text { spanned by vector } \mathbf{w}\end{array}$ \\
\hline $\begin{array}{l}\text { Control space } \\
K \times 1 \text { Vector }\end{array}$ & $\begin{array}{l}\alpha, \beta \\
\chi^{\mathbb{J}^{+}} \\
\chi^{\mathbb{J}^{-}}\end{array}$ & $\begin{array}{l}\text { Control vectors } \\
\text { Control vector whose } k^{\text {th }} \text { entry is } \chi^{+}\left(\mathbf{u}_{\mathbb{J}}^{T} \mathbf{p}_{k}\right) \\
\text { Control vectors whose } k^{\text {th }} \text { entry is } \chi^{-}\left(\mathbf{u}_{\mathbb{J}}^{T} \mathbf{p}_{k}\right)\end{array}$ \\
\hline $\begin{array}{l}\text { Functions } \\
\mathbb{R} \rightarrow\{0,1\} \\
\mathbb{R}^{3} \rightarrow \mathbb{R} \\
\mathcal{G} \rightarrow[0,1]^{K}\end{array}$ & $\begin{aligned} \chi^{-}(\cdot) / \chi^{+}(\cdot) \\
\\
\quad \mathscr{V}(\mathcal{G}) \\
\quad \mathscr{C}(\mathbf{t})\end{aligned}$ & $\begin{array}{l}\text { Negative/positive indicator functions } \\
\text { Volume of } \mathcal{G} \\
\text { Color Control Function }(\mathrm{CCF})\end{array}$ \\
\hline
\end{tabular}

\section{A. Sets, Vectors, Combinations, Indicator Functions}

We denote the empty set by $\phi$. For a positive integer $n$ and a nonnegative integer $k, k \leq n$, the standard notation $\left(\begin{array}{l}n \\ k\end{array}\right)$ is used to denote the number $n ! /(k !(n-k) !)$ of possible combinations with $k$ elements that can be selected from $n$ distinct objects. For any positive integer $M$, we denote by $\langle M\rangle$ the set $\{1,2,3, \ldots, M\}$ of integers from 1 through $M$. We use a singleton integer set and its integer element interchangeably. Also, when not explicitly defined as a sequence, if required, we equivalently consider a set of integers as a sequence with the elements arranged in increasing order. Given a set of integer indices $\mathbb{I}$, we denote the set of all possible permutations of elements of $\mathbb{I}$ by $\mathfrak{P}(\mathbb{I})$, and for any integer $m, 0 \leq m \leq \mathscr{N}(\mathbb{I})$, we denote the set of all possible combinations of $m$ elements chosen from $\mathbb{I}$ by $\mathfrak{C}^{m}(\mathbb{I})$, where $\mathscr{N}(\mathbb{I})$ denotes the cardinality of the set $\mathbb{I}$. It follows immediately that $\mathscr{N}\left(\mathfrak{C}^{m}(\mathbb{I})\right)=\left(\begin{array}{c}\mathscr{N} \mathbb{I}) \\ m\end{array}\right)$ and $\mathscr{N}(\mathfrak{P}(\mathbb{I}))=\mathscr{N}(\mathbb{I})$ !. Given two sets $\mathbb{I}$ and $\mathbb{I}$ of indices, we denote by $\mathbb{I} \backslash \mathbb{J}$ the set of indices in $\mathbb{I}$ not contained in $\mathbb{J}$, also referred to as the difference between the sets (in that order).

Standard notation is used for matrices and vectors, with boldface upper-case letters representing matrices and boldface lower-case letters representing vectors. Transposes for vectors and matrices are denoted by the superscript ${ }^{T}$. The set of real numbers is denoted by $\mathbb{R}$ and the $\ell^{2}$-norm of a vector $\mathbf{x} \in \mathbb{R}^{N}$ is denoted by $\|\mathbf{x}\|=\sqrt{\mathbf{x}^{T} \mathbf{x}}$. For any $N \times M$ matrix A and a sequence (set) of indices $\mathbb{I} \subset\langle M\rangle$ we denote by $\mathbf{A}[\mathbb{I}]$ the submatrix of $\mathbf{A}$ formed by the selection of the columns indexed by $\mathbb{I}$ in that sequence. We denote the $M \times M$ identity matrix by $\mathbf{I}_{M}=\left[\mathbf{e}_{1}^{M}, \mathbf{e}_{2}^{M}, \ldots \mathbf{e}_{N}^{M}\right]$, where $\mathbf{e}_{i}^{M}$ denotes the $i^{\text {th }}$ column of $\mathbf{I}_{M}$. Then using our afore-mentioned convention for indexing columns $\mathbf{I}_{M}[[i, j, k]]$ denotes the $M \times 3$ matrix that has a 1 in the $i^{\text {th }}, j^{\text {th }}$, and $k^{\text {th }}$ rows in the first, second, and third columns, respectively, and other entries as zeros. For an ordered basis $\mathbf{b}_{1}, \mathbf{b}_{2}, \ldots, \mathbf{b}_{N}$ for $\mathbb{R}^{N}$, the orientation is said to be positive or negative based on whether the determinant of the matrix $\mathbf{B}=\left[\mathbf{b}_{1}, \mathbf{b}_{2}, \ldots, \mathbf{b}_{N}\right]$ is positive or negative, respectively ${ }^{1}$. Specifically, in $\mathbb{R}^{3}$ the right-hand rule applies to an ordered basis with a positive orientation. For a $N \times 2$ matrix $\mathbf{B}=\left[\mathbf{b}_{1}, \mathbf{b}_{2}\right]$, with $\mathbf{b}_{1}$ and $\mathbf{b}_{2}$ denoting the columns, we define $\otimes \mathbf{B} \equiv \mathbf{b}_{1} \otimes \mathbf{b}_{2}$, where $\otimes$ denotes the vector cross-product following the right-hand-rule convention. If $\mathbf{x}, \mathbf{y}, \mathbf{z} \in \mathbb{R}^{3}$, we have det $([\mathbf{x}, \mathbf{y}, \mathbf{z}])=(\mathbf{x} \otimes \mathbf{y})^{T} \mathbf{z}=(\mathbf{y} \otimes \mathbf{z})^{T} \mathbf{x}=(\mathbf{z} \otimes \mathbf{x})^{T} \mathbf{y}=$ $-(\mathbf{x} \otimes \mathbf{z})^{T} \mathbf{y}$.

We define the positivity indicator function $\chi^{+}: \mathbb{R} \rightarrow\{0,1\}$ as $\chi^{+}(x)=1$, for $x>0$, and $\chi^{+}(x)=0$, otherwise. The negativity indicator function is then defined by $\chi^{-}(x)=\chi^{+}(-x)$ and we use $\operatorname{sgn}(x)=\chi^{+}(x)-\chi^{-}(x)$ to denote the sign of $x$. For $x, y \in \mathbb{R}$ with $x<y$ we denote by $[x, y]$ and $(x, y)$, respectively, the closed and open intervals of real numbers between $x$ and $y$.

\section{B. Affine Subspaces, Interior, Exterior, Boundary}

For any $M<N$ linearly independent vectors $\mathbf{b}_{1}, \mathbf{b}_{2}, \ldots, \mathbf{b}_{M} \in \mathbb{R}^{N}$ and any vector $\mathbf{v} \in \mathbb{R}^{N}$, the set $\mathcal{U}=$

\footnotetext{
${ }^{1}$ Our definition for orientation of a basis corresponds with notion of orientation of a vector space [14], [15]. Specifically, a basis with positive orientation maintains the same vector space orientation as the ordered natural basis, which is represented by the matrix $\mathbf{I}_{N}$.
} 
$\mathcal{U}(\mathbf{v}, \mathbf{B})=\left\{\mathbf{B x}+\mathbf{v} \mid \mathbf{x} \in \mathbb{R}^{M}\right\}$ defines an $M$-dimensional affine subspace of $\mathbb{R}^{N}$, where $\mathbf{B}=\left[\mathbf{b}_{1}, \mathbf{b}_{2}, \ldots, \mathbf{b}_{M}\right]$ and conversely such a representation exists for any $M$ dimensional affine subspace of $\mathbb{R}^{N}$. We denote the open ball in $\mathcal{U}$ centered at $\mathbf{x}$ with radius $r$ by $\mathcal{D}_{\mathcal{U}}(\mathbf{x}, r)=\{\mathbf{t} \in \mathcal{U} \mid\|\mathbf{t}-\mathbf{x}\|<r\}$, or by $\mathcal{D}(\mathbf{x}, r)$ when $\mathcal{U}$ is $\mathbb{R}^{N}$.

For any set $\mathcal{A} \subset \mathbb{R}^{N}$, let $\mathcal{U}_{\mathcal{A}}$ be the affine subspace of minimum dimensionality, $d$, that contains $\mathcal{A}$. Then we say that $\mathcal{A}$ has dimension $d$ and that a point $\mathrm{x}$ is in the interior ${ }^{2}$ of a set $\mathcal{A}$ if there exists a $\delta>0$ such that $\mathcal{D}_{\mathcal{U}_{\mathcal{A}}}(\mathbf{x}, \delta) \subset \mathcal{A}$. Conversely, we say a point $\mathrm{x}$ is exterior to the set $\mathcal{A}$ if there exists a $\delta>0$ such that $\mathcal{D}_{\mathcal{U}_{\mathcal{A}}}(\mathbf{x}, \delta) \subset \mathcal{A}^{c}$, where $\mathcal{A}^{c}=\mathcal{U}_{\mathcal{A}} \backslash \mathcal{A}$ denotes the complement of $\mathcal{A}$ in $\mathcal{U}_{\mathcal{A}}$. We denote by $\operatorname{Int}(\mathcal{A})$ the set of all points in the interior of the set $\mathcal{A}$. The boundary $\mathcal{B}_{\mathcal{A}}$ of a set $\mathcal{A}$ is defined as the set of points that are neither in the interior of nor the exterior of the set. One can readily see that any point $\mathbf{t} \in \mathcal{U}_{\mathcal{A}}$ lies in the boundary $\mathcal{B}_{\mathcal{A}}$ of $\mathcal{A}$ if and only if (iff) $\mathcal{D}_{\mathcal{U}_{\mathcal{A}}}(\mathbf{t}, \delta) \bigcap \mathcal{A} \neq \phi$ and $\mathcal{D}_{\mathcal{U}_{\mathcal{A}}}(\mathbf{t}, \delta) \cap \mathcal{A}^{c} \neq \phi$ for all $\delta>0$. For a closed set, it is relatively straightforward to see that the boundary lies within the set, whereas for an open set, the boundary is outside the set. For three dimensional sets with a finite nonzero volume, we refer to the boundary interchangeably as the surface. We say two sets $\mathcal{X}, \mathcal{Y}$ contained in a common minimum dimensionality affine subspace $\mathcal{U}$ are essentially-disjoint if their interiors have a null intersection ${ }^{3}$, i.e., $\operatorname{Int}(\mathcal{X}) \bigcap \operatorname{Int}(\mathcal{Y})=\phi$.

\section{Convexity and Polytopes}

Given $m$ points $\mathbf{x}_{1}, \mathbf{x}_{2}, \ldots \mathbf{x}_{m} \in \mathbb{R}^{N}$, a linear combination of the form $\sum_{i=1}^{m} \xi_{i} \mathbf{x}_{i}$, where $\xi_{i} \in[0,1], i=1,2, \ldots m$ and $\sum_{i=1}^{m} \xi_{i}=1$, is referred to as a convex combination of the points $\mathbf{x}_{1}, \mathbf{x}_{2}, \ldots \mathbf{x}_{m}$. A polytope in $\mathbb{R}^{N}$ is the convex hull, i.e., the set of all convex combinations, of a finite set of points in $\mathbb{R}^{N}$. The extremal points in a convex set are the points in the set that cannot be expressed as convex linear combinations of other points in the set. The extremal points in a polytope are referred to as the vertices of the polytope. A polytope is identical to the convex hull of its vertices, which is the unique minimal representation for the polytope in the sense that any other representation as a convex hull uses a superset of the vertices. The set $\overline{\mathcal{H}}(\mathbf{w}, v)=\left\{\mathbf{x} \mid \mathbf{w}^{T} \mathbf{x}=v\right\}$ denotes a hyperplane, where $\mathbf{w}$ is a unit normal to the plane and $v$ is the (signed) distance of the plane to the origin along the unit normal. Corresponding to the hyperplane, two closed halfspaces on its either side are $\mathcal{H}^{-}(\mathbf{w}, v)=\left\{\mathbf{x} \mid \mathbf{w}^{T} \mathbf{x} \leq v\right\}$ and $\mathcal{H}^{+}(\mathbf{w}, v)=\left\{\mathbf{x} \mid \mathbf{w}^{T} \mathbf{x} \geq v\right\}$. The intersection $\mathcal{Q}=$ $\bigcap_{i} \mathcal{H}^{*}\left(\mathbf{w}_{i}, v_{i}\right)$ of a finite number of closed half-spaces is referred to as a polyhedral set, where $\mathcal{H}^{*}\left(\mathbf{w}_{i}, v_{i}\right)$ is either $\mathcal{H}^{-}\left(\mathbf{w}_{i}, v_{i}\right)$ or $\mathcal{H}^{+}\left(\mathbf{w}_{i}, v_{i}\right)$. A polytope is a bounded polyhedral set and every bounded polyhedral set is a polytope [16, pp. 119-120]. For a convex set $\mathcal{A} \subset \mathbb{R}^{N}$, a hyperplane

\footnotetext{
${ }^{2}$ Technically, our definitions of interior and exterior correspond, respectively, to relative-interior and relative-exterior with respect to the minimum dimensionality affine subspace $\mathcal{U}_{\mathcal{A}}$ that contains the set $\mathcal{A}$. The minimum dimensionality affine subspace containing a set will be implicit based on the context and we omit the "relative" qualifier for brevity.

${ }^{3}$ In $\mathbb{R}^{3}$, two non-zero volume sets are essentially-disjoint iff their intersection has a zero volume.
}

$\overline{\mathcal{H}}(\mathbf{w}, v)$ is said to be a supporting hyperplane if there exists a point $\mathbf{x} \in \mathcal{A} \cap \overline{\mathcal{H}}(\mathbf{w}, v)$ and either $\mathcal{A} \subset \mathcal{H}^{-}(\mathbf{w}, v)$ or $\mathcal{A} \subset \mathcal{H}^{+}(\mathbf{w}, v)$. If $\overline{\mathcal{H}}(\mathbf{w}, v)$ is a supporting-hyperplane for a polytope $\mathcal{Q}, \mathcal{C}=\mathcal{Q} \cap \overline{\mathcal{H}}(\mathbf{w}, v)$ is itself a polytope lying on the boundary of $\mathcal{Q}$, and is referred to as a $l$-face, where $l$ is the is the dimensionality of the face. In addition to these proper faces, $\mathcal{Q}$ and $\phi$ are referred to as improper faces of $\mathcal{Q}$. The vertices of any face of a polytope $\mathcal{Q}$ are the vertices of the polytope that lie within the face. Any intersection of faces of a polytope is also a face. The 0 -faces of a polytope are its vertices and the 1-faces are referred to as edges. For a $d$-dimensional polytope, $(d-1)$ faces are referred to as its facets, which collectively form the entire boundary of the polytope and every $(d-2)$-face is contained in precisely two facets and is the intersection of the two facets that contain it [13, pp. 34-35]. For a polytope in $\mathbb{R}^{3}$, the facets, edges, and vertices comprise the full set of non-empty faces. Furthermore, if $\mathcal{F}_{i}, i=1,2, \ldots N_{f}$ denote the facets for a polytope $\mathcal{Q}$, with $N_{f}$ denoting their number, and $\overline{\mathcal{H}}\left(\mathbf{w}, v_{i}\right)$ are the associated hyperplanes and $\mathcal{H}^{*}\left(\mathbf{w}, v_{i}\right)$ are the associated half-spaces (in the same order) such that $\mathcal{Q} \subset \mathcal{H}^{*}\left(\mathbf{w}, v_{i}\right)$, then $\mathcal{Q}=\bigcap_{i=1}^{N_{f}} \mathcal{H}^{*}\left(\mathbf{w}, v_{i}\right)$ and $\mathcal{F}_{i}=\mathcal{Q} \bigcap \overline{\mathcal{H}}\left(\mathbf{w}, v_{i}\right)[16$, pp. 119]. For a unit vector $\mathbf{w} \in \mathbb{R}^{3}, \tau, \nu \in \mathbb{R}, \tau<\nu$, we define the set

$\mathcal{H}(\mathbf{w}, \tau, \nu) \equiv \mathcal{H}^{+}(\mathbf{w}, \tau) \bigcap \mathcal{H}^{-}(\mathbf{w}, \nu)=\left\{\mathbf{t} \in \mathbb{R}^{3} \mid \tau \leq \mathbf{w}^{T} \mathbf{t} \leq \nu\right\}$,

as the region in $\mathbb{R}^{3}$ lying between two parallel hyperplanes $\overline{\mathcal{H}}(\mathbf{w}, \tau)$ and $\overline{\mathcal{H}}(\mathbf{w}, \nu)$. We refer to $\mathcal{H}(\mathbf{w}, \tau, \nu)$ as the $\mathbf{w}$ plane slice, with extent between $\tau$ and $\nu$. Note that the normal w points into and out-of the plane slice $\mathcal{H}(\mathbf{w}, \tau, \nu)$ at the bounding planes $\overline{\mathcal{H}}(\mathbf{w}, \tau)$ and $\overline{\mathcal{H}}(\mathbf{w}, \nu)$, respectively.

\section{Display Model, Gamut, and Color Control FUNCTION}

Consider a display system that produces colors using a set of $K$ light sources that are referred to as the display primaries and characterized by their spectral (radiance) power distributions $p_{k}(\lambda), k=1, \ldots, K$. Colors are produced by additively combining these primaries after non-spectrally-selective attenuation. Specifically, we assume that a $K \times 1$ control vector $\boldsymbol{\alpha} \stackrel{\text { def }}{=}\left[\alpha_{1}, \alpha_{2}, \ldots, \alpha_{K}\right]^{T} \in[0,1]^{K}$ controls the scaling of the individual primaries and produces a corresponding displayed spectral power distribution

$$
s(\boldsymbol{\alpha}, \lambda)=\sum_{k=1}^{K} \alpha_{k} p_{k}(\lambda)+s_{0}(\lambda),
$$

where $s_{0}(\lambda)$ is the display black spectral radiance, which is the display emission when $\boldsymbol{\alpha}=\mathbf{0}$. A corresponding representation in CIE XYZ ${ }^{4}$ tristimulus color space [1] follows immediately from the observation that the tristimulus vector $\mathbf{t} \in \mathbb{R}^{3}$ corresponding to a spectral power distribution $s(\boldsymbol{\alpha}, \lambda)$ is defined as

$$
\mathbf{t}(\boldsymbol{\alpha})=\left[\begin{array}{c}
t_{X} \\
t_{Y} \\
t_{Z}
\end{array}\right] \stackrel{\text { def }}{=}\left[\begin{array}{c}
\int \bar{x}(\lambda) s(\boldsymbol{\alpha}, \lambda) d \lambda \\
\int \bar{y}(\lambda) s(\boldsymbol{\alpha}, \lambda) d \lambda \\
\int \bar{z}(\lambda) s(\boldsymbol{\alpha}, \lambda) d \lambda
\end{array}\right]
$$

\footnotetext{
${ }^{4}$ Other additive tristimulus color spaces may equivalently be used.
} 
where $\bar{x}(\lambda), \bar{y}(\lambda)$, and $\bar{z}(\lambda)$ are the CIE XYZ color matching functions [1]. Equivalently, the tristimulus corresponding to the control vector $\boldsymbol{\alpha}$ is given by

$$
\mathbf{t}(\boldsymbol{\alpha})=\sum_{k=1}^{K} \alpha_{k} \mathbf{p}_{k}+\mathbf{t}_{\mathbf{0}}=\mathbf{P} \boldsymbol{\alpha}+\mathbf{t}_{\mathbf{0}}
$$

where $\mathbf{p}_{k}$ denotes the tristimulus for the $k^{\text {th }}$ primary, $\mathbf{P}=$ $\left[\mathbf{p}_{1}, \mathbf{p}_{2}, \ldots \mathbf{p}_{K}\right]$ is the matrix of primary tristimulus values, and $\mathbf{t}_{\mathbf{0}}$ is the display black tristimulus, and $\mathbf{t}_{\mathbf{1}} \stackrel{\text { def }}{=} \mathbf{t}_{\mathbf{0}}+$ $\sum_{k=1}^{K} \mathbf{p}_{k}$, is the display white tristimulus obtained when all primaries are turned completely on. Throughout our development, we assume that any subset of three primaries chosen from $\mathbf{P}$ is linearly independent ${ }^{5}$. The model in (1) and (3) provides reasonable agreement with common color display technologies [6].

The range

$$
\begin{aligned}
\mathcal{G} & =\left\{\mathbf{t}(\boldsymbol{\alpha}) \mid \boldsymbol{\alpha} \in[0,1]^{K}\right\} \\
& =\left\{\mathbf{P} \boldsymbol{\alpha}+\mathbf{t}_{\mathbf{0}} \mid \boldsymbol{\alpha} \in[0,1]^{K}\right\}
\end{aligned}
$$

of tristimulus values that the display can produce is called the gamut (in CIE XYZ space). From the preceding definition, it is apparent that the gamut $\mathcal{G}$ is the image of the unit hypercube $[0,1]^{K}$ under an affine transformation. Because the $[0,1]^{K}$ is a convex compact set in $\mathbb{R}^{K}$, it therefore follows that $\mathcal{G}$ is also a convex and compact set.

The forward model in (3) provides the tristimulus that the display produces given the control vector $\boldsymbol{\alpha}$. When using the display, one is typically more interested in an inverse for the model, which, given a tristimulus $\mathbf{t} \in \mathcal{G}$, determines a control vector $\boldsymbol{\alpha}(\mathbf{t})$ such that the display reproduces the tristimulus $\mathbf{t}$ in response to the control vector $\boldsymbol{\alpha}(\mathbf{t})$. A function that assigns a control vector to every tristimulus in the gamut is referred to as a color control function (CCF). Formally, a CCF is a function $\mathscr{C}: \mathcal{G} \rightarrow[0,1]^{K}$ such that for each $\mathbf{t} \in \mathcal{G}, \mathscr{C}(\mathbf{t})$ is a control vector for the tristimulus t.

It is readily apparent that the gamut $\mathcal{G}$ in (4) is an affine map of the $K$-dimensional unit hypercube in $\mathbb{R}^{K}$ into $\mathbb{R}^{3}$, where $K \geq 3$. In general, an affine map of the $K$-dimensional unit hypercube in $\mathbb{R}^{K}$ into $\mathbb{R}^{N}$ is called a zonotope [10, Chap. 7], which, as we already indicated, is a specific type of convex polytope. The gamut zonotope, however, is further constrained to being 3 dimensional, generated by vectors (primaries) that from physical constraints contain only nonnegative components, and satisfy the condition that any subset of three vectors is linearly independent. In our development in the next section, these distinguishing characteristics of the gamut zonotope allows us to provide a more complete and readily usable mathematical characterization of the gamut zonotope than is currently available for general zonotopes. We also note that color control functions do not naturally arise in the context of general polytopes but are related to zonotopal tilings. We remark on this relation after presenting our main results in the

\footnotetext{
${ }^{5}$ In practice, this constraint is met, except in degenerate scenarios that are not particularly of interest because there exist infinitesimally small perturbations that dispel the degeneracy while causing only infinitesimal changes in any other quantities of interest.
}

next section and develop this further in the companion Part II paper [12].

\section{GAmut Representations And Color Control FUNCTIONS}

For our development, we introduce a series of sets that represent generalizations of the gamut definition in (4) and represent the span of sets of tristimulus values under the control value bounds. Specifically, for $j \geq 1$, given a $3 \times 1$ vector $\mathbf{v}$ and a $3 \times j$ matrix $\mathbf{A}=\left[\mathbf{a}_{1}, \mathbf{a}_{2}, \ldots, \mathbf{a}_{j}\right]$, where any subset of three or fewer columns of $\mathbf{A}$ are linearly independent, we denote by

$$
\begin{aligned}
\mathcal{S}(\mathbf{v}, \mathbf{A}) & \equiv\left\{\sum_{i=1}^{j} \alpha_{i} \mathbf{a}_{i}+\mathbf{v} \mid 0 \leq \alpha_{i} \leq 1, i=1,2, \ldots j\right\} \\
& \equiv\left\{\mathbf{A} \boldsymbol{\alpha}+\mathbf{v} \mid \boldsymbol{\alpha} \in[0,1]^{j}\right\}
\end{aligned}
$$

the region of $\mathbb{R}^{3}$ spanned by $\mathbf{A}$ under the control vector bounds $^{6}$ at the origin $\mathbf{v}$. One can immediately see that for $j \leq 2, \mathcal{U}(\mathbf{v}, \mathbf{A})$ is the affine subspace of minimum dimensionality, $j$, that contains $\mathcal{S}(\mathbf{v}, \mathbf{A})$, and for $j \geq 3, \mathbb{R}^{3}$ is the (affine) subspace of minimum dimensionality that contains $\mathcal{S}(\mathbf{v}, \mathbf{A})$.

It follows that the gamut $\mathcal{G}$ can alternatively be written as $\mathcal{G}=\mathcal{S}\left(\mathbf{t}_{\mathbf{0}}, \mathbf{P}\right)$. Three other instances of (5) play important roles in the geometry of gamuts and, for this reason, we explicitly call these out here. For a $3 \times 1$ tristimulus vector $\mathbf{v}$ and a $3 \times 3$ matrix $\mathbf{A}=\left[\mathbf{a}_{1}, \mathbf{a}_{2}, \mathbf{a}_{3}\right]$, where the columns $\mathbf{a}_{1}, \mathbf{a}_{2}, \mathbf{a}_{3}$ of $\mathbf{A}$ are three linearly independent tristimulus vectors, we denote by $\mathcal{P}(\mathbf{v}, \mathbf{A}) \equiv \mathcal{S}(\mathbf{v}, \mathbf{A})$ the parallelepiped spanned by $\mathbf{A}$ at origin $\mathbf{v}$. Similarly, for a $3 \times 1$ tristimulus vector $\mathbf{v}$ and a $3 \times 2$ matrix $\mathbf{B}=\left[\mathbf{b}_{1}, \mathbf{b}_{2}\right]$, where the columns $\mathbf{b}_{1}, \mathbf{b}_{2}$ of $\mathbf{B}$ are two linearly independent tristimulus vectors, we denote by $\mathcal{F}(\mathbf{v}, \mathbf{B}) \equiv \mathcal{S}(\mathbf{v}, \mathbf{B})$ the parallelogram spanned by $\mathbf{B}$ at origin $\mathbf{v}$. Finally, for a pair of $3 \times 1$ tristimulus vectors $\mathbf{v}$ and $\mathbf{c}$, we denote by $\mathcal{E}(\mathbf{v}, \mathbf{c}) \equiv \mathcal{S}(\mathbf{v}, \mathbf{c})$ the line segment spanned by $\mathbf{c}$ at origin $\mathbf{v}$.

The parallelepiped $\mathcal{P}(\mathbf{v}, \mathbf{A})$, in particular, is the fundamental building block for our constructions and we therefore establish several key properties of this set in the following lemma.

Lemma 1. Let $\mathbf{v}$ be a $3 \times 1$ vector and $\mathbf{A}=\left[\mathbf{a}_{1}, \mathbf{a}_{2}, \mathbf{a}_{3}\right]$ be a $3 \times 3$ matrix of linearly independent tristimulus vectors $\mathbf{a}_{1}, \mathbf{a}_{2}, \mathbf{a}_{3}$. For each $\mathbb{J} \in \mathfrak{C}^{2}(\langle 3\rangle)$, let $i=\langle 3\rangle \backslash \mathbb{J}$ and denote ${ }^{7}$

$$
\begin{aligned}
\mathbf{u}_{\mathbb{J}} & =\frac{1}{\|(\otimes \mathbf{A}[\mathbb{J}])\|}(\otimes \mathbf{A}[\mathbb{J}]), \\
\mathbf{d}^{\mathbb{J}^{-}} & =\mathbf{v}+\chi^{-}\left(\mathbf{u}_{\mathbb{J}}^{T} \mathbf{a}_{i}\right) \mathbf{a}_{i} \\
\mathbf{d}^{\mathbb{J}^{+}} & =\mathbf{v}+\chi^{+}\left(\mathbf{u}_{\mathbb{J}}^{T} \mathbf{a}_{i}\right) \mathbf{a}_{i} \\
\tau^{\mathbb{J}} & =\mathbf{u}_{\mathbb{J}}^{T} \mathbf{d}^{\mathbb{J}^{-}}, \\
\nu^{\mathbb{J}} & =\mathbf{u}_{\mathbb{J}}^{T} \mathbf{d}^{\mathbb{J}^{+}} .
\end{aligned}
$$

\section{Then}

\footnotetext{
${ }^{6}$ Throughout this paper, the term "spanned" will implicitly carry the qualification of being defined by the $[0,1]$ control vector bounds instead of the more traditional definition of span which is not subject to bounds.

${ }^{7}$ The entries in $\mathbb{J}$ are arranged in increasing order by our adopted convention, which makes the definitions unambiguous.
} 
1) The parallelepiped $\mathcal{P} \stackrel{\text { def }}{=} \mathcal{P}(\mathbf{v}, \mathbf{A})$ is a polytope that can be represented as the intersection of plane slices, as

$$
\mathcal{P}=\bigcap_{\mathbb{J} \in \mathfrak{C}^{2}(\langle 3\rangle)} \mathcal{H}\left(\mathbf{u}_{\mathbb{J}}, \tau^{\mathbb{J}}, \nu^{\mathbb{J}}\right) .
$$

2) The surface of $\mathcal{P}$ can be expressed as the union of six distinct facets as

$$
\mathcal{B}_{\mathcal{P}}=\bigcup_{\mathbb{J} \in \mathfrak{C}^{2}(\langle 3\rangle)}\left(\mathcal{F}\left(\mathbf{d}^{\mathbb{J}^{-}}, \mathbf{A}[\mathbb{J}]\right) \cup \mathcal{F}\left(\mathbf{d}^{\mathbb{J}^{+}}, \mathbf{A}[\mathbb{J}]\right)\right) .
$$

For each $\mathbb{J} \in \mathfrak{C}^{2}(\langle 3\rangle), \mathcal{F}\left(\mathbf{d}^{\mathbb{J}^{-}}, \mathbf{A}[\mathbb{J}]\right)$ and $\mathcal{F}\left(\mathbf{d}^{\mathbb{J}^{+}}, \mathbf{A}[\mathbb{J}]\right)$ represent a pair of congruent parallelogram facets with supporting planes $\overline{\mathcal{H}}\left(\mathbf{u}_{\mathbb{J}}, \tau^{\mathbb{J}}\right)$ and $\overline{\mathcal{H}}\left(\mathbf{u}_{\mathbb{J}}, \nu^{\mathbb{J}}\right)$. Specifically,

$$
\begin{aligned}
\mathcal{F} & \left(\mathbf{d}^{\mathbb{J}^{-}}, \mathbf{A}[\mathbb{J}]\right)=\mathcal{P} \bigcap \overline{\mathcal{H}}\left(\mathbf{u}_{\mathbb{J}}, \tau^{\mathbb{J}}\right) \\
& =\overline{\mathcal{H}}\left(\mathbf{u}_{\mathbb{J}}, \tau^{\mathbb{J}}\right) \bigcap\left(\bigcap_{\mathbb{J}^{\prime} \in \mathfrak{C}^{2}(\langle 3\rangle) \backslash \mathbb{J}} \mathcal{H}\left(\mathbf{u}_{\mathbb{J}^{\prime}}, \tau^{\mathbb{J}^{\prime}}, \nu^{\mathbb{J}^{\prime}}\right)\right), \\
\mathcal{F} & \left(\mathbf{d}^{\mathbb{J}^{+}}, \mathbf{A}[\mathbb{J}]\right)=\mathcal{P} \bigcap \overline{\mathcal{H}}\left(\mathbf{u}_{\mathbb{J}}, \nu^{\mathbb{J}}\right) \\
& =\overline{\mathcal{H}}\left(\mathbf{u}_{\mathbb{J}}, \nu^{\mathbb{J}}\right) \bigcap\left(\bigcap_{\mathbb{J}^{\prime} \in \mathfrak{C}^{2}(\langle 3\rangle) \backslash \mathbb{J}} \mathcal{H}\left(\mathbf{u}_{\mathbb{J}^{\prime}}, \tau^{\mathbb{J}^{\prime}}, \nu^{\mathbb{J}^{\prime}}\right)\right) .
\end{aligned}
$$

Each edge of a facet spanned by a vector $\mathbf{a}_{j}$ is supported by a plane $\overline{\mathcal{H}}\left(\mathbf{u}_{\mathbb{J}}, \tau^{\mathbb{J}}\right)$ or $\overline{\mathcal{H}}\left(\mathbf{u}_{\mathbb{J}}, \nu^{\mathbb{J}}\right)$ for some $\mathbb{J} \in \mathfrak{C}^{2}(\langle 3\rangle)$ such that $j \in \mathbb{J}$.

3) The edges of $\mathcal{P}$ are distinctly enumerated as the union

$$
\begin{aligned}
& \mathcal{W}_{\mathcal{P}}=\bigcup_{\mathbb{J} \in \mathfrak{C}^{2}(\langle 3\rangle)}\left\{\mathcal{E}\left(\mathbf{d}^{\mathbb{J}^{-}}, \mathbf{a}_{\mathbb{J}[1]}\right), \mathcal{E}\left(\mathbf{d}^{\mathbb{J}^{-}}+\mathbf{a}_{\mathbb{J}[1]}, \mathbf{a}_{\mathbb{J}[2]}\right),\right.
\end{aligned}
$$

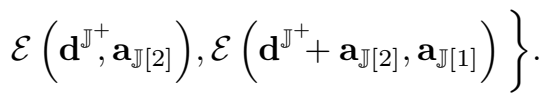

Thus the parallelepiped has 12 edges.

4) The vertices of the parallelepiped $\mathcal{P}$ are

$$
\mathcal{V}_{\mathcal{P}}=\bigcup_{\mathbb{J} \in \mathfrak{C}^{2}(\langle 3\rangle)}\left\{\mathbf{d}^{\mathbb{J}^{-}}+\mathbf{a}_{\mathbb{I}[1]}, \mathbf{d}^{\mathbb{J}^{+}}+\mathbf{a}_{\mathbb{J}[2]}\right\} \bigcup\left\{\mathbf{v}, \mathbf{v}+\sum_{l=1}^{3} \mathbf{a}_{l}\right\},
$$

where each listed vertex is unique. Hence the parallelepiped has a total of 8 vertices. For each $\mathbb{J} \in \mathfrak{C}^{2}(\langle 3\rangle)$, the points $\mathbf{d}^{\mathbb{J}^{-}}+\mathbf{a}_{\mathbb{J}[1]}$ and $\mathbf{d}^{\mathbb{J}^{+}}+\mathbf{a}_{\mathbb{J}[2]}$ are vertices of the facets $\mathcal{F}\left(\mathbf{d}^{\mathbb{J}^{-}}, \mathbf{A}[\mathbb{J}]\right)$ and $\mathcal{F}\left(\mathbf{d}^{\mathbb{I}^{+}}, \mathbf{A}[\mathbb{J}]\right)$, respectively.

5) The linear, bijective (and continuous) function defined by the mapping $\mathbf{t} \mapsto \mathbf{A}^{-1}(\mathbf{t}-\mathbf{v}) \stackrel{\text { def }}{=} \boldsymbol{\beta}(\mathbf{t})$ for each $\mathbf{t} \in \mathbb{R}^{3}$ maps $\mathcal{P}$ onto $[0,1]^{3}$. Thus, for any $\mathbf{t} \in \mathcal{P}$, $\boldsymbol{\alpha}=\mathbf{A}^{-1}(\mathbf{t}-\mathbf{v}) \in[0,1]^{3}$ is the unique feasible control vector such that $\mathbf{t}=\mathbf{A} \boldsymbol{\alpha}+\mathbf{v}$. Furthermore, for $i=1,2,3$, $\beta_{i}(\mathbf{t})=\left((-1)^{(i+1)} / \operatorname{det}(\mathbf{A})\right)(\otimes \mathbf{A}[\mathbb{J}])^{T}(\mathbf{t}-\mathbf{v})$.

The proof of Lemma 1 is straightforward using basic linear algebra. For completeness, it is provided as Appendix A.

Lemma 1 establishes that the surface of $\mathcal{P}(\mathbf{v}, \mathbf{A})$ is comprised of three pairs of opposing congruent parallelograms that form the facets of $\mathcal{P}(\mathbf{v}, \mathbf{A})$. Thus $\mathcal{P}(\mathbf{v}, \mathbf{A})$ is in fact a parallelepiped [16], which will be one of the fundamental building blocks in our representations of gamuts. Figure 1 illustrates an example of the parallelepiped $\mathcal{P}(\mathbf{v}, \mathbf{A})$ and its key geometrical elements defined in Lemma 1 for a specific case $^{8}$ where $\mathbf{v}=[35,35,35]^{T}$, and

$$
\begin{aligned}
& \mathbf{A}=\left[\begin{array}{rrr}
0.0259 & 1.7448 & 118.6868 \\
20.3238 & 85.4870 & 3.6479 \\
114.6842 & 58.1553 & 22.7756
\end{array}\right] \\
& \stackrel{\text { def }}{=}\left[\mathbf{a}_{1}, \mathbf{a}_{2}, \mathbf{a}_{3}\right] .
\end{aligned}
$$

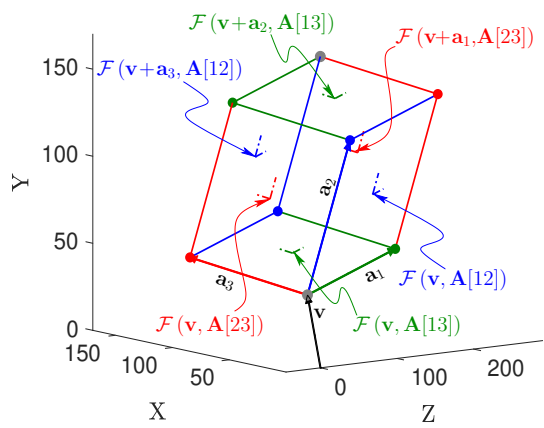

(a)

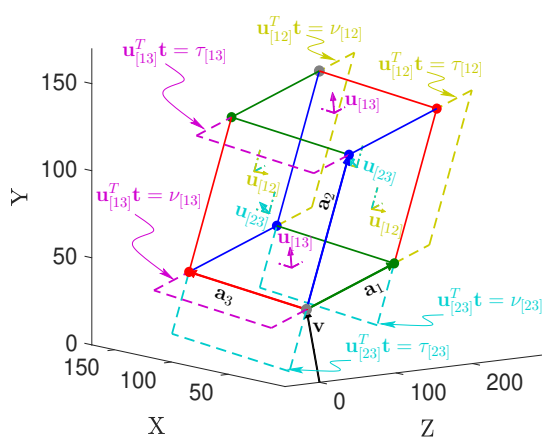

(b)

Fig. 1. Geometric elements of the parallelepiped $\mathcal{P}(\mathbf{v}, \mathbf{A})$ identified by Lemma 1 for an example with $\mathbf{v}=[35,35,35]^{T}$ and $\mathbf{A}$ as defined in (16): (a) the parallelogram facets $\mathcal{F}\left(\mathbf{d}^{\mathbb{J}^{-}}, \mathbf{A}[\mathbb{J}]\right), \mathcal{F}\left(\mathbf{d}^{\mathbb{J}^{+}}, \mathbf{A}[\mathbb{J}]\right)$ that form the surface (boundary) of the parallelepiped $\mathcal{P}(\mathbf{v}, \mathbf{A})$. Pairs of opposing parallelogram facets are labeled in text of identical color and the edges and vertices associated with the facets are also shown in the corresponding colors, (b) edges of the parallelepiped $\mathcal{P}(\mathbf{v}, \mathbf{A})$, shown in gray with the facets extended and labeled to illustrate the pairs of parallel planes $\mathbf{u}_{\mathfrak{J}}^{T} \mathbf{t}=\tau^{\mathbb{J}}$ and $\mathbf{u}_{\mathbb{J}}^{T} \mathbf{t}=\nu^{\mathbb{J}}$ that determine the plane slices $\mathcal{H}\left(\mathbf{u}_{\mathbb{J}}, \tau^{\mathbb{J}}, \nu^{\mathbb{J}}\right)$. Normal vectors $\mathbf{u}_{\mathbb{J}}$ are shown for the planes.

For $K=3$ primaries, we immediately see that $\mathcal{G}=$ $\mathcal{P}\left(\mathbf{t}_{\mathbf{0}}, \mathbf{P}\right)$. Thus the tristimulus gamut for a three primary system is a parallelepiped. For this commonly encountered scenario, Lemma 1 completely characterizes the tristimulus gamut by (a) providing two alternative gamut representations, specifically as a parallelepiped bounded by surface parallelograms and as a region of tristimulus space, defined by three pairs of bounding planes, (b) uniquely enumerates all the facets, edges, and vertices of the gamut, and (c) establishes the uniqueness of the control vector for each ingamut color $\mathbf{t} \in \mathcal{G}$ and therefore of the (display) $\mathrm{CCF} \mathscr{C}$. For a multiprimary display, the following theorem, that builds upon

\footnotetext{
${ }^{8}$ The synthetic set of primaries is chosen purely for making geometric illustrations clearer in this and subsequent examples.
} 
and generalizes Lemma 1, provides analogous representations for the gamut.

Theorem 1. Consider the $K$ primary display defined in Section III by the $3 \times K$ matrix of (nonnegative) primaries $\mathbf{P}=\left[\mathbf{p}_{1}, \mathbf{p}_{2}, \ldots, \mathbf{p}_{K}\right]$ and the display black tristimulus $\mathbf{t}_{\mathbf{0}}$ with gamut $\mathcal{G}$ defined in (4), where $K \geq 3$ and any selection of three columns from $\mathbf{P}$ is linearly independent. For each $\mathbb{J} \in \mathfrak{C}^{2}(\langle K\rangle)$, define:

$$
\begin{aligned}
\mathbf{u}_{\mathbb{J}} & =\frac{1}{\|(\otimes \mathbf{P}[\mathbb{J}])\|}(\otimes \mathbf{P}[\mathbb{J}]), \\
\chi^{\mathbb{J}^{-}} & =\left[\chi^{-}\left(\mathbf{u}_{\mathbb{J}}^{T} \mathbf{p}_{1}\right), \chi^{-}\left(\mathbf{u}_{\mathbb{J}}^{T} \mathbf{p}_{2}\right), \ldots, \chi^{-}\left(\mathbf{u}_{\mathbb{J}}^{T} \mathbf{p}_{K}\right)\right]^{T}, \\
\chi^{\mathbb{J}^{+}} & =\left[\chi^{+}\left(\mathbf{u}_{\mathbb{J}}^{T} \mathbf{p}_{1}\right), \chi^{+}\left(\mathbf{u}_{\mathbb{J}}^{T} \mathbf{p}_{2}\right), \ldots, \chi^{+}\left(\mathbf{u}_{\mathbb{J}}^{T} \mathbf{p}_{K}\right)\right]^{T}, \\
\mathbf{d}^{\mathbb{J}^{-}} & =\mathbf{t}_{\mathbf{0}}+\mathbf{P} \chi^{\mathbb{J}^{-}}, \\
\mathbf{d}^{\mathbb{J}^{+}} & =\mathbf{t}_{\mathbf{0}}+\mathbf{P} \chi^{\mathbb{J}^{+}}, \\
\tau^{\mathbb{J}} & =\mathbf{u}_{\mathbb{J}}^{T} \mathbf{d}^{\mathbb{J}^{-}}, \\
\nu^{\mathbb{J}} & =\mathbf{u}_{\mathbb{J}}^{T} \mathbf{d}^{\mathbb{J}^{+}},
\end{aligned}
$$

and, additionally, for $l=1,2, \ldots K$, define $K \times 1$ vectors $\chi_{l}^{\mathbb{J}^{+}}$ and $\chi_{l}^{\mathbb{J}^{-}}$that have the first $l$ entries identical to $\chi^{\mathbb{J}^{+}}$and $\chi^{\mathbb{J}^{-}}$, respectively, and remaining entries as 0 . Then,

1) The gamut $\mathcal{G}$ can be represented as an essentially-disjoint union of $\left(\begin{array}{c}K \\ 3\end{array}\right)$ parallelepipeds as

$$
\begin{aligned}
\mathcal{G} & =\bigcup_{i=3}^{K}\left(\bigcup_{\mathbb{J} \in \mathfrak{C}^{2}(\langle i-1\rangle)} \mathcal{P}\left(\mathbf{c}_{i}^{\mathbb{J}}, \mathbf{P}[\mathbb{J}, i]\right)\right), \text { where } \\
\mathbf{c}_{i}^{\mathbb{J}} & =\mathbf{t}_{\mathbf{0}}+\mathbf{P} \boldsymbol{\alpha}_{i}^{\mathbb{J}}, \\
\boldsymbol{\alpha}_{i}^{\mathbb{J}} & =\chi^{+}\left(\mathbf{u}_{\mathbb{J}}^{T} \mathbf{p}_{i}\right) \chi_{i-1}^{\mathbb{J}^{+}}+\chi^{-}\left(\mathbf{u}_{\mathbb{J}}^{T} \mathbf{p}_{i}\right) \chi_{i-1}^{\mathbb{J}^{-}} .
\end{aligned}
$$

2) The gamut is a polytope that can alternatively be represented as the intersection of plane slices

$$
\mathcal{G}=\bigcap_{\mathbb{J} \in \mathfrak{C}^{2}(\langle K\rangle)} \mathcal{H}\left(\mathbf{u}_{\mathbb{J}}, \tau^{\mathbb{J}}, \nu^{\mathbb{J}}\right)
$$

3) The surface of $\mathcal{G}$ can be expressed as the union of distinct facets as

$$
\mathcal{B}_{\mathcal{G}}=\bigcup_{\mathbb{J} \in \mathfrak{C}^{2}(\langle K\rangle)}\left(\mathcal{F}\left(\mathbf{d}^{\mathbb{J}^{-}}, \mathbf{P}[\mathbb{J}]\right) \cup \mathcal{F}\left(\mathbf{d}^{\mathbb{J}^{+}}, \mathbf{P}[\mathbb{J}]\right)\right),
$$

For each $\mathbb{J} \in \mathfrak{C}^{2}(\langle K\rangle), \mathcal{F}\left(\mathbf{d}^{\mathbb{J}^{-}}, \mathbf{P}[\mathbb{J}]\right)$ and $\mathcal{F}\left(\mathbf{d}^{\mathbb{J}^{+}}, \mathbf{P}[\mathbb{J}]\right)$ represent a pair of congruent parallelogram facets with supporting planes $\overline{\mathcal{H}}\left(\mathbf{u}_{\mathbb{J}}, \tau^{\mathbb{J}}\right)$ and $\overline{\mathcal{H}}\left(\mathbf{u}_{\mathbb{J}}, \nu^{\mathbb{J}}\right)$. Specifically,

$$
\begin{aligned}
& \mathcal{F}\left(\mathbf{d}^{\mathbb{J}^{-}}, \mathbf{P}[\mathbb{J}]\right)=\mathcal{G} \bigcap \overline{\mathcal{H}}\left(\mathbf{u}_{\mathbb{J}}, \tau^{\mathbb{J}}\right), \\
& \mathcal{F}\left(\mathbf{d}^{\mathbb{J}^{+}}, \mathbf{P}[\mathbb{J}]\right)=\mathcal{G} \bigcap \overline{\mathcal{H}}\left(\mathbf{u}_{\mathbb{J}}, \nu^{\mathbb{J}}\right) .
\end{aligned}
$$

Thus there are $\left(\begin{array}{c}K \\ 2\end{array}\right)$ pairs of parallelogram facets, i.e., $K(K-1)$ facets. Each edge of a facet spanned by a primary $\mathbf{p}_{j}$ is supported by a plane $\overline{\mathcal{H}}\left(\mathbf{u}_{\bar{J}}, \tau^{\bar{J}}\right)$ or $\overline{\mathcal{H}}\left(\mathbf{u}_{\overline{\mathbb{J}}}, \nu^{\overline{\mathbb{J}}}\right)$ for some $\overline{\mathbb{J}} \in \mathfrak{C}^{2}(\langle K\rangle)$ such that $j \in \overline{\mathbb{J}}$.

4) The edges of the gamut $\mathcal{G}$ are given by

$$
\begin{aligned}
& \mathcal{W}_{\mathcal{G}}=\bigcup_{\mathbb{J} \in \mathfrak{C}^{2}(\langle K\rangle)}\left\{\mathcal{E}\left(\mathbf{d}^{\mathbb{J}^{-}}, \mathbf{p}_{\mathbb{J}[1]}\right), \mathcal{E}\left(\mathbf{d}^{\mathbb{J}^{-}}+\mathbf{p}_{\mathbb{J}[1]}, \mathbf{p}_{\mathbb{J}[2]}\right),\right. \\
& \left.\mathcal{E}\left(\mathbf{d}^{\mathbb{J}^{+}}, \mathbf{p}_{\mathbb{J}[2]}\right), \mathcal{E}\left(\mathbf{d}^{\mathbb{J}^{+}+} \mathbf{p}_{\mathbb{J}[2]}, \mathbf{p}_{\mathbb{J}[1]}\right)\right\},
\end{aligned}
$$

where each listed edge is distinct. Thus the gamut has $2 K(K-1)$ edges.

5) The vertices of the gamut polytope $\mathcal{G}$ are

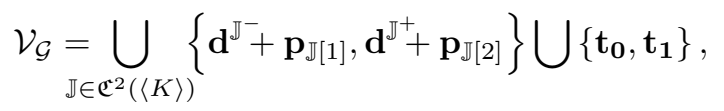

where each listed vertex is unique. Hence the gamut has a total of $K(K-1)+2$ vertices.

6) For a $K \geq 4$ primary display, there is a unique control vector for a tristimulus $\mathbf{t} \in \mathcal{G}$, iff $\mathbf{t} \in \mathcal{B}_{\mathcal{G}}$.

7) A CCF $\mathscr{C}$ that is continuous and piece-wise linear over each of the parallelepiped partitions in the representation in (24) is obtained by defining, for $i=3,4, \ldots, K$ and for all $\mathbb{J} \in \mathfrak{C}^{2}([i-1])$,

$$
\mathscr{C}(\mathbf{t})=\boldsymbol{\alpha}_{i}^{\mathbb{J}}+\mathbf{I}_{K}[[\mathbb{J}, i]] \mathbf{P}^{-1}[\mathbb{J}, i]\left(\mathbf{t}-\mathbf{c}_{i}^{\mathbb{J}}\right),
$$

for all $\mathbf{t} \in \mathcal{P}\left(\mathbf{c}_{i}^{\mathbb{J}}, \mathbf{P}[\mathbb{J}, i]\right)$, where $\boldsymbol{\alpha}_{i}^{\mathbb{J}}$ is as defined in (26). This is the unique CCF function associated with the gamut representation of (24), or equivalently, with the given order for the primaries, in the sense that for each $i$ and each $\mathbb{J} \in \mathfrak{C}^{2}([i-1])$, the CCF for each tristimulus $\mathbf{t} \in \mathcal{P}\left(\mathbf{c}_{i}^{\mathbb{J}}, \mathbf{P}[\mathbb{J}, i]\right)$ is defined by a color control vector of the form $\boldsymbol{\alpha}(\mathbf{t})=\boldsymbol{\alpha}_{i}^{\mathbb{J}}+\boldsymbol{\delta}^{\mathbb{J}, i}(\mathbf{t})$, where $\delta_{l}^{(\mathbb{J}, i)}(\mathbf{t})=0$ for $l \notin[\mathbb{J}, i]$ and $0 \leq \delta_{l}^{(\mathbb{J}, i)}(\mathbf{t}) \leq 1$ for $l \in[\mathbb{J}, i]$.

8) For any two parallelepipeds in (24) that have a nonempty intersection, the intersection is a proper face of both parallelepipeds.

9) For each $\mathbb{J} \in \mathfrak{C}^{2}(\langle K\rangle)$, the $(K-2)$ parallelepipeds in (24) that include the primaries $\mathbf{P}[\mathbb{J}]$ form a sequence where the intersection of successive parallelepipeds is a common facet of both parallelepipeds spanned by the primaries $\mathbf{P}[\mathbb{J}]$, i.e., there exists $\mathbb{I} \in \mathfrak{P}(\langle K\rangle \backslash \mathbb{J})$ such that for any $1 \leq l \leq(K-3)$,

$$
\begin{aligned}
& \mathcal{P}\left(\mathbf{c}_{\mathbb{I}[l]}^{\mathbb{J}}, \mathbf{P}[\mathbb{J} \mid \mathbb{I}[l]]\right) \bigcap \overline{\mathcal{H}}\left(\mathbf{u}_{\mathbb{J}}, \bar{\eta}_{\mathbb{I}[l]}^{\mathbb{J}}\right)= \\
& \mathcal{P}\left(\mathbf{c}_{\mathbb{I}[l+1]}^{\mathbb{J}}, \mathbf{P}[\mathbb{J} \mid \mathbb{I}[l+1]]\right) \bigcap \overline{\mathcal{H}}\left(\mathbf{u}_{\mathbb{J}}, \bar{\kappa}_{\mathbb{I}[l+1]}^{\mathbb{J}}\right),
\end{aligned}
$$

where $\bar{\eta}_{\mathbb{I}[l]}^{\mathbb{J}}=\mathbf{u}_{\mathbb{J}}^{T}\left(\mathbf{c}_{\mathbb{I}[l]}^{\mathbb{J}}+\chi^{+}\left(\mathbf{u}_{\mathbb{J}}^{T} \mathbf{p}_{\mathbb{I}[l]}\right) \mathbf{p}_{\mathbb{I}[l]}\right)=\bar{\kappa}_{\mathbb{I}[l+1]}^{\mathbb{J}}=$ $\mathbf{u}_{\mathbb{J}}^{T}\left(\mathbf{c}_{\mathbb{I}[l]}^{\mathbb{J}}+\chi^{-}\left(\mathbf{u}_{\mathbb{J}}^{T} \mathbf{p}_{\mathbb{I}[l+1]}\right) \mathbf{p}_{\mathbb{I}[l+1]}\right)$.

The proof for Theorem 1 is provided in Appendix B. Theorem 1 immediately provides a direct and readily usable method for obtaining the complete representation of the gamut of a multiprimary display and the associated CCF in (33). Algorithm 1 outlines the simple computational procedure for computation of relevant parameters for the gamut surface and 
the parallelepipeds in the gamut tiling ${ }^{9}$ of (24), where we have introduced the short-hand arg sort $\uparrow$ to return the list of indices that sorts the argument in increasing order. Once these parameters have been computed, all the facets, edges, and vertices can be uniquely and exhaustively enumerated using (28), (31), and (32), respectively, to obtain the complete representation of the gamut boundary. We note that although Algorithm 1 obtains these gamut and CCF representations for a $K$ primary gamut directly, without iterating on the number of primaries $K$, the sequence of the primaries in the primary matrix $\mathbf{P}$ does determine the parallelepiped tiling in (24), its associated CCF in (33), and the parallelepiped chains in Clause 9 associated with the pairs of opposing gamut facets in (28) for each $\mathbb{J} \in \mathfrak{C}^{2}(\langle K\rangle)$. We therefore refer to the representation in (24) as a progressive-by-primary gamut tiling and the associated CCF $\mathscr{C}(\cdot)$ in (33) as the corresponding progressive-by-primary tiling $C C F$.

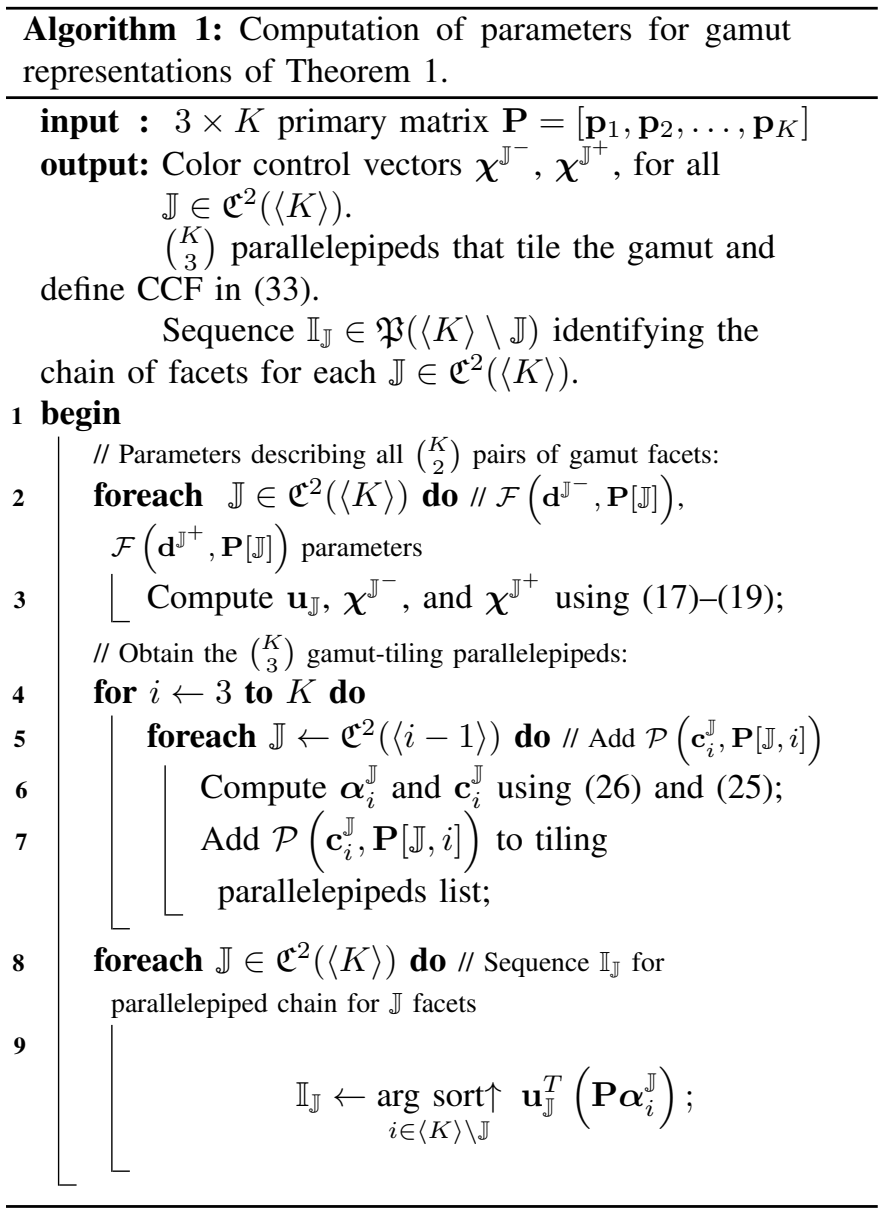

We illustrate the gamut representations obtained using Theorem 1 via an example, using $\mathbf{t}_{\mathbf{0}}=[35,35,35]^{T}$ and the four primary matrix

$$
\begin{aligned}
& \mathbf{P}=\left[\mathbf{A} \mid[86.475,76.533,31.177]^{T}\right] \\
& \stackrel{\text { def }}{=}\left[\mathbf{p}_{1}, \mathbf{p}_{2}, \mathbf{p}_{3}, \mathbf{p}_{4}\right]
\end{aligned}
$$

\footnotetext{
${ }^{9}$ Here we use the term tiling without a formal definition, which is subsequently provided in the Part II companion paper [12].
}

which is an extension of the three primary system defined by matrix $\mathbf{A}$ in equation (16). Figure 2 illustrates the tristimulus gamut $\mathcal{G}$ for this four primary system, where the edges and vertices of the gamut are colored to indicate the associations as per Clauses 4 and 5 of Theorem 1. Specifically, the two edges of the same color in a facet indicate the edges associated with the facet through (31) and the vertex located at the intersection of these edges is the vertex that (32) associates with the same facet. It can be appreciated that, as required by Theorem 1, in each of the cases, (31) and (32), respectively, enumerate the edges and vertices, exhaustively and uniquely. The corresponding progressive-by-primary tiling of the gamut following Algorithm 1 is shown in Fig. 3 (a)-(d), where the progression of figures illustrates the progressive nature of the

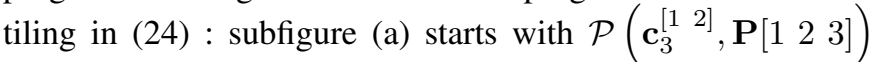
(the only parallelepiped for $i=3$ ), and subfigures (b)(d) then introduce, one by one, all the three parallelepipeds spanned by $\mathbf{p}_{4}$, a parallelepiped for each of the pairs of facets

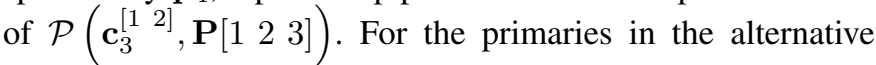
permuted order $\check{\mathbf{P}} \stackrel{\text { def }}{=}\left[\check{\mathbf{p}}_{1}, \check{\mathbf{p}}_{2}, \check{\mathbf{p}}_{3}, \check{\mathbf{p}}_{4}\right]=\left[\mathbf{p}_{1}, \mathbf{p}_{4}, \mathbf{p}_{3}, \mathbf{p}_{2}\right]$, a different progressive-by-primary tiling of the same gamut is obtained, as shown in Fig. 3 (e)-(h), where the difference between the tilings can be particularly appreciated by comparing subfigures 3 (d) and (h). The different tilings also induce different CCFs, which we denote as $\mathscr{C}_{\mathbf{P}}$ and $\mathscr{C}_{\tilde{\mathbf{P}}}$, respectively. In Figs. 3 (c) and (g) two tristimuli $\mathbf{t}_{a}$ (green star) and $\mathbf{t}_{b}$ (red star) located, respectively, in the interior and on the surface of the gamut are also illustrated, which are obtained with control values $\mathscr{C}_{\mathbf{P}}\left(\mathbf{t}_{a}\right)=[1 / 3,1,2 / 3,2 / 3]^{T}$ and $\mathscr{C}_{\mathbf{P}}\left(\mathbf{t}_{b}\right)=[2 / 3,1,1 / 3,1]^{T}$ for the CCF $\mathscr{C}_{\mathbf{P}}$ and have the associated control values $\mathscr{C}_{\mathbf{P}}\left(\mathbf{t}_{a}\right)=[0.45,1,0.49,0.68]^{T}$ and $\mathscr{C}_{\mathbf{P}}\left(\mathbf{t}_{b}\right)=[2 / 3,1,1 / 3,1]^{T}$ for the $\operatorname{CCF} \mathscr{C}_{\mathbf{P}}$. Note that, the two CCFs yield different control values for $\mathbf{t}_{a}$ in the interior of the gamut and the same control values for the tristimulus $\mathbf{t}_{b}$ on the gamut surface (as mandated by Clause 6 of Theorem 1 and also by the equivalent result in [11]).

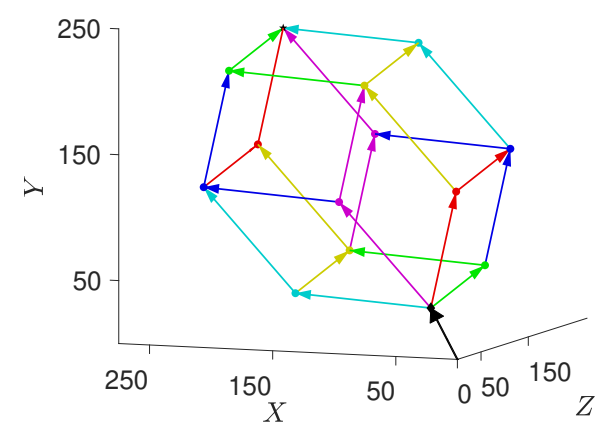

Fig. 2. The tristimulus gamuts $\mathcal{G}$ for the $K=4$ primary systems $\mathbf{P}$ defined in (35). The region inside the wireframe corresponds to the gamut and the edges and vertices are colored to indicate the associations as per Clauses 4 and 5 of Theorem 1 .

Additional visualizations of example five and six multiprimary display tristimulus gamuts that illustrate the general applicability and utility of Theorem 1 are included in Appendix D. For a multiprimary display with a given set of primaries, $K$ ! (not necessarily distinct) progressive-by- 
primary tilings and associated CCFs can be obtained using Algorithm 1, by arranging the primaries in all possible orderings. Appendix E illustrates the distinct progressive-byprimary tilings obtained using Algorithm 1 for a $K=5$ primary system. A natural question arises whether and how the set of all possible control values for each tristimulus, which we refer to as the metameric control set (MCS) for the tristimulus, relates to the alternative CCFs associated with the progressiveby-primary tilings. As it turns out, the alternative progressiveby-primary CCFs, provide only a partial characterization of the MCS and only a subset of possible parallelepiped tiling CCFs. In the Part II companion paper [12], we address this topic and provide a complete characterization. The methodology connects closely with the parallelepiped chains described in Clause 9 of Theorem 1.

\section{ApPLiCATiON EXAMPLES}

We now present a series of examples illustrating how Theorem 1 and Algorithm 1 provide useful tools for the visualization of gamuts, gamut mapping, for the design of primaries, and the construction of CCFs with advantageous attributes.

\section{A. Perceptual Gamut Boundary for Visualization and Gamut Mapping}

Practical uses of multiprimary displays demand color management strategies for reproducing imagery that is originally designed for the gamut of alternative color devices [17]. Such strategies usually include gamut mapping operations [18], whose design can benefit from the description of the gamut surface, facets, edges, and vertices introduced in this paper. To allow for trade-offs to be meaningfully formulated based on the application requirements, gamut mapping is invariably performed in a color appearance space where the axes and distances are semantically meangingful. A perceptual representation of the tristimulus $\mathbf{t}$ is obtained as the mapping of $\mathbf{t}$ to a perceptual space by a nonlinear transformation $\mathscr{F}_{\mathbf{t}_{1}}(\cdot)$, where the explicit dependence of the transformation on the white tristimulus $\mathbf{t}_{\mathbf{1}}$ indicates the fact that human color vision adapts its response relative to the white. The perceptual representation for tristimulus $\mathbf{t}$ is denoted by the $3 \times 1$ vector $\boldsymbol{\tau}$, and obtained as $\boldsymbol{\tau} \stackrel{\text { def }}{=}\left[\tau_{L}, \tau_{c_{1}}, \tau_{c_{2}}\right]^{T}=\mathscr{F}_{\mathbf{t}_{1}}(\mathbf{t})$, where the $\tau_{L}$ represents the perceptual correlate of lightness, and $\tau_{c_{1}}, \tau_{c_{2}}$ represent the perceptual correlates of red-green and yellow-blue chroma, respectively. The perceptual gamut $\mathcal{G}_{\mathscr{F}} \stackrel{\text { def }}{=} \mathscr{F}_{\mathbf{t}_{\mathbf{1}}}(\mathcal{G})=\left\{\boldsymbol{\tau} \mid \boldsymbol{\tau}=\mathscr{F}_{\mathbf{t}_{\mathbf{1}}}\left(\mathbf{P} \boldsymbol{\alpha}+\mathbf{t}_{\mathbf{0}}\right), \boldsymbol{\alpha} \in[0,1]^{K}\right\}$ is then the representation of reproducible colors in the perceptual color space. Since $\mathscr{F}_{\mathbf{t}_{1}}(\cdot)$ is a continuous one-to-one mapping, using (24), $\mathcal{G}_{\mathscr{F}}$ can alternatively be expressed as the essentially-disjoint union

$$
\mathcal{G}_{\mathscr{F}}=\bigcup_{i=3}^{K}\left(\bigcup_{\mathbb{J} \in \mathfrak{C}^{2}(\langle i-1\rangle)} \mathscr{F}_{\mathbf{t}_{\mathbf{1}}}\left(\mathcal{P}\left(\mathbf{c}_{i}^{\mathbb{J}}, \mathbf{P}[\mathbb{J}, i]\right)\right)\right),
$$

while $\mathcal{B}_{\mathcal{G}_{\mathscr{F}}} \stackrel{\text { def }}{=} \mathscr{F}_{\mathbf{t}_{\mathbf{1}}}\left(\mathcal{B}_{\mathcal{G}}\right)$, the surface of $\mathcal{G}_{\mathscr{F}}$, is expressed as

$$
\mathcal{B}_{\mathcal{G}_{\mathscr{F}}}=\bigcup_{\mathbb{J} \in \mathfrak{C}^{2}(\langle K\rangle)}\left(\bigcup_{\mathbf{d}^{\mathbb{J} *} \in\left\{\mathbf{d}^{\mathbb{J}}, \mathbf{d}^{\mathbb{J}+}\right\}} \mathscr{F}_{\mathbf{t}_{1}}\left(\mathcal{F}\left(\mathbf{d}^{\mathbb{J}^{*}, \mathbf{P}[\mathbb{J}]}\right)\right)\right) .
$$

Figure 4 shows the perceptual gamut $\mathcal{G}_{\mathscr{F}}$ (via a plot of its boundary $\mathcal{B}_{\mathcal{G}_{\mathscr{F}}}$ ) in the CIELUV [1] perceptual color space ${ }^{10}$ for the four primary system $\mathbf{P}_{\mathscr{V}}^{(4)}$ specified in Appendix C, which was designed to optimize the volume of $\mathcal{G}_{\mathscr{F}}$ under a total optical power constraint (as discussed later in this section). The description of the perceptual gamut boundary in (37), as well as the perceptual representation of the gamut edges and vertices, can be exploited for the efficient computation and visualization of specific regions of the boundary, such as loci of constant hue (hue slices) or constant lightness, which are used in mapping strategies that best preserve perceptual attributes of color [18], [21], [22].

\section{B. Gamut Volume and Gamut Inclusion for Primary De- sign/Evaluation}

The geometric representations of the gamut introduced in this paper allow for the computation of alternative metrics traditionally used for primary designs: the volume of the perceptual gamut [23]-[25] and the fraction of real-world surface colors that are included within the gamut [26], [27]. Theorem 1 allows for both these metrics to be readily computed as we illustrate next.

The representation of the tristimulus gamut $\mathcal{G}$ and the perceptual gamut $\mathcal{G}_{\mathscr{F}}$ in (24) and (36), respectively, partition the gamut into essentially-disjoint sets: $\mathcal{G}$ is tiled by a set of parallelepipeds $\mathcal{P}(\cdot)$, whose perceptual representations $\mathscr{F}_{\mathbf{t}_{1}}(\mathcal{P}(\cdot))$ yield an essentially disjoint partition of $\mathcal{G}_{\mathscr{F}}$. This geometrical representation can be conveniently exploited for the computation of the gamut volume in both tristimulus and perceptual color spaces. In particular, using the fact that the volume of the parallelepiped spanned by a set of three primaries is the absolute value of the determinant of the $3 \times 3$ matrix with the primaries as its columns [28, pp. 468], it directly follows from (24) that the volume of the tristimulus gamut $\mathcal{G}$ is

$$
\mathscr{V}(\mathcal{G})=\sum_{i=3}^{K}\left(\sum_{\mathbb{J} \in \mathfrak{C}^{2}([i-1])}|\operatorname{det}(\mathbf{P}[\mathbb{J}, i])|\right) .
$$

Similarly, the volume of $\mathcal{G}_{\mathscr{F}}, \mathscr{V}\left(\mathcal{G}_{\mathscr{F}}\right)=\int_{\mathcal{G}_{\mathscr{F}}} d \boldsymbol{\tau}$, can be expressed using (36) as the summation

$$
\mathscr{V}\left(\mathcal{G}_{\mathscr{F}}\right)=\sum_{i=3}^{K}\left(\sum_{\mathbb{J} \in \mathfrak{C}^{2}([i-1])} \mathscr{V}\left(\mathscr{F}_{\mathbf{t}_{1}}\left(\mathcal{P}\left(\mathbf{c}_{i}^{\mathbb{J}}, \mathbf{P}[\mathbb{J}, i]\right)\right)\right)\right),
$$

where $\mathscr{V}\left(\mathscr{F}_{\mathbf{t}_{1}}\left(\mathcal{P}\left(\mathbf{c}_{i}^{\mathbb{J}}, \mathbf{P}[\mathbb{J}, i]\right)\right)\right)$ is the volume of the perceptual representation of $\mathcal{P}\left(\mathbf{c}_{i}^{\mathbb{J}}, \mathbf{P}[\mathbb{J}, i]\right)$, which can be computed using efficient numerical strategies, as proposed in [7].

\footnotetext{
${ }^{10}$ The methodology can be readily utilized for most color appearance spaces, including, current state of the art color appearance spaces such as CAM16 [19]. We use the CIELUV space for simplicity and prefer it over CIELAB because the optimization formulations we present subsequently have made use of CIELUV due to challenges with CIELAB in the dark regions [20].
} 


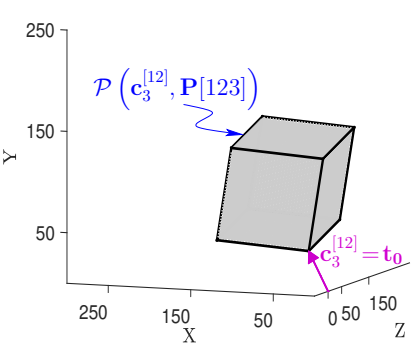

(a) $i=3, \mathbb{J}=\left[\begin{array}{ll}1 & 2\end{array}\right]$

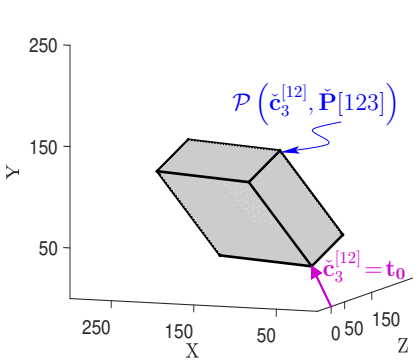

(e) $\check{i}=3, \check{\mathbb{J}}=\left[\begin{array}{ll}1 & 2\end{array}\right]$

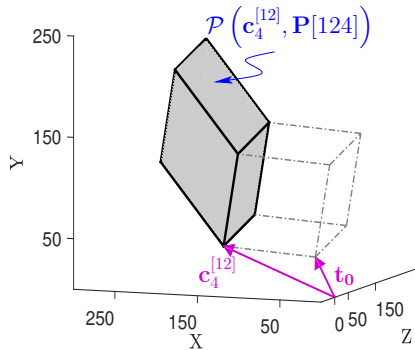

(b) $i=4, \mathbb{J}=\left[\begin{array}{ll}1 & 2\end{array}\right]$

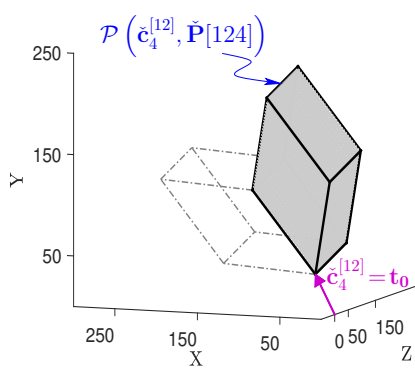

(f) $\breve{i}=4, \check{\mathbb{J}}=\left[\begin{array}{ll}1 & 2\end{array}\right]$

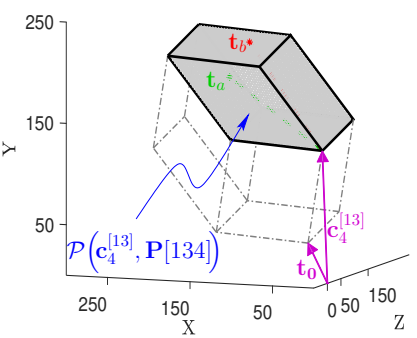

(c) $i=4, \mathbb{J}=\left[\begin{array}{ll}1 & 3\end{array}\right]$

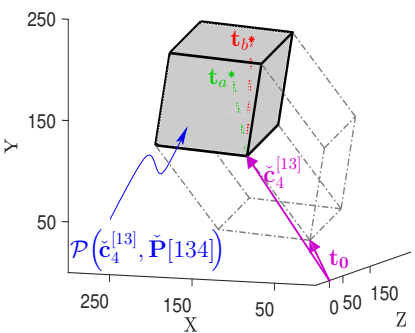

(g) $\check{i}=4, \check{\mathbb{J}}=\left[\begin{array}{ll}1 & 3\end{array}\right]$

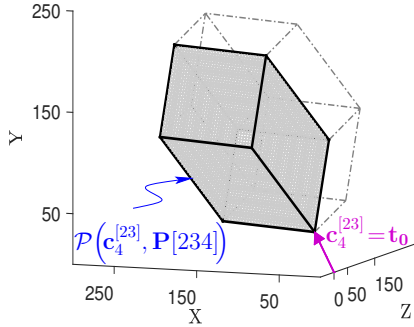

(d) $i=4, \mathbb{J}=\left[\begin{array}{ll}2 & 3\end{array}\right]$

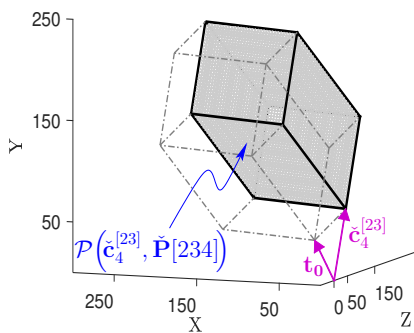

(h) $\check{i}=4, \check{J}=\left[\begin{array}{ll}2 & 3\end{array}\right]$

Fig. 3. Progressive-by-primary gamut tilings for the four primary display systems with primary matrices $\mathbf{P}=\left[\mathbf{p}_{1}, \mathbf{p}_{2}, \mathbf{p}_{3}, \mathbf{p}_{4}\right] \check{\mathbf{P}}^{\text {def }}=\left[\check{\mathbf{p}}_{1}, \check{\mathbf{p}}_{2}, \check{\mathbf{p}}_{3}, \check{\mathbf{p}}_{4}\right]=$ $\left[\mathbf{p}_{1}, \mathbf{p}_{4}, \mathbf{p}_{3}, \mathbf{p}_{2}\right]$, obtained from Algorithm 1. Also illustrated are two tristimuli $\mathbf{t}_{a}$ (green star) and $\mathbf{t}_{b}$ (red star) located in the interior and on the surface of the gamut for which the progressive-by-primary CCFs corresponding to the tilings provide, respectively, different and identical control values (See text).

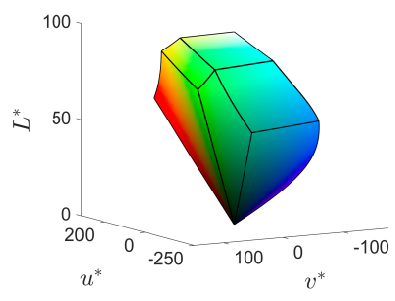

(a)

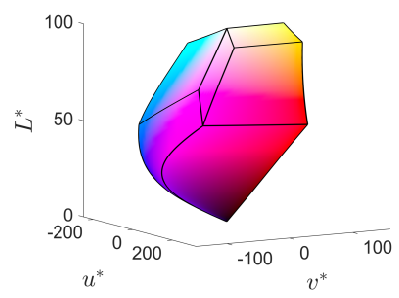

(c)

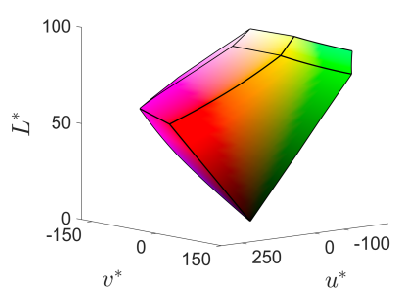

(b)

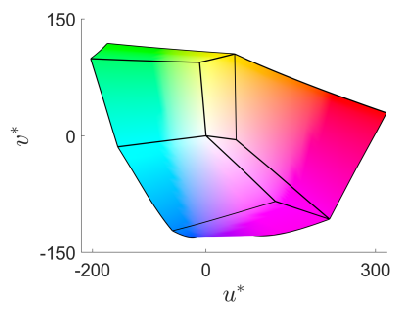

(d)
Fig. 4. The perceptual gamut $\mathcal{G}_{\mathscr{F}}$ in CIELUV space for the four primary system $\mathbf{P}_{\mathscr{V}}^{(4)}$ designed to maximize the perceptual gamut volume using the methodology in [23]. Figures (a)-(d) show $\mathcal{G}_{\mathscr{F}}$ from different perspectives. The (CIELUV representations of) edges of $\mathcal{G}$ identified in (31) are plotted as black lines.

Table II, shows the volume ${ }^{11}$ for tristimulus and perceptual gamuts for optimal configurations of $K=3,4$, and 5 primaries designed to maximize perceptual volume under a total power constraint, following the methodology proposed in [23]. The

\footnotetext{
${ }^{11}$ To enable meaningful comparison of tristimulus volumes, primaries were normalized to have a luminance of 100 for the white. Perceptual gamut volumes are in units of $\left(\Delta E_{u v}^{*}\right)^{3}$.
}

specifications of the optimal primaries are provided in Appendix C. As a comparison baseline, Table II also includes the corresponding volumes for the primary specifications of the High Definition TV, REC. 709 [29] and Ultra High Definition TV, REC. 2020 [30] standards.

A display's capability for reproducing real-world surface colors is usually quantified by a coverage ratio defined as the fraction $\mathscr{R}_{\mathcal{A}}(\mathcal{G}) \stackrel{\text { def }}{=} \mathscr{N}(\mathcal{G} \cap \mathcal{A}) / \mathscr{N}(\mathcal{A})$ of the set of representative real-world surface color tristimuli $\mathcal{A}$ that are included in the display gamut $\mathcal{G}$. From the representation of the gamut as the intersection of plane slices in (27), we see that $\mathcal{G} \cap \mathcal{A}=\left\{\mathbf{t} \in \mathcal{A} \mid \tau^{\mathbb{J}} \leq \mathbf{u}_{\mathbb{J}}^{T} \mathbf{t} \leq \nu^{\mathbb{J}}, \forall \mathbb{J} \in \mathfrak{C}^{2}(\langle K\rangle)\right\}$, therefore, $\mathscr{N}(\mathcal{G} \cap \mathcal{A})$ is efficiently obtained by the counting the number of elements in $\mathcal{A}$ that satisfy the $K(K-1)$ inequalities. For the optimized and standard primary configurations, Table II also shows the coverage ratios $\mathscr{R}_{\text {Pointer }}$ and $\mathscr{R}_{\text {SOCS }}$, respectively, for two sets of surface colors that have frequently been used for evaluating primary designs: the Pointer set of surface colors [31] and the SOCS database [32],

\begin{tabular}{|c|c|c|c|c|c|c|}
\hline & \multicolumn{4}{|c|}{$\begin{array}{l}\text { Optimized Primaries } \\
\quad\left(\max . \mathscr{V}\left(\mathcal{G}_{\mathscr{F}}\right)\right)\end{array}$} & \multicolumn{2}{|c|}{$\begin{array}{c}\text { Standard Primaries } \\
(K=3)\end{array}$} \\
\hline & $K=3$ & $K=4$ & $K=5$ & $K=6$ & $\begin{array}{l}\text { REC709 } \\
\end{array}$ & REC2020 \\
\hline $\mathscr{V}(\mathcal{G})$ & 499944 & 526554 & 537517 & 540526 & 207094 & 418070 \\
\hline $\mathscr{V}\left(\mathcal{G}_{\mathscr{F}}\right)$ & 3034764 & 3254984 & 3331988 & 3365340 & 1327110 & 2531126 \\
\hline $\mathscr{R}_{\text {Pointer }}$ & 0.91 & 0.98 & 0.98 & 0.99 & 0.52 & 1.00 \\
\hline $\mathscr{R}_{\text {SOCS }}$ & 0.99 & 1.00 & 1.00 & 1.00 & 0.89 & 1.00 \\
\hline
\end{tabular}

GAMUT VOLUME AND COVERAGE RATIO FOR OPTIMIZED $K=3,4,5$, AND 6 PRIMARY DISPLAY SYSTEMS AND FOR COMMON STANDARDS. 


\section{CCF Design: The Axially Linear CCF}

In [33], we demonstrated that a CCF that is linear along lines of constant chromaticity has the advantageous property that the display gray axis is perceptually robust to variations in the primaries (under the reasonable assumption that the entries in the display black tristimulus $\mathbf{t}_{\mathbf{0}}$ are negligible, i.e. $\approx \mathbf{0}$, compared to those in display white tristimulus $\mathbf{t}_{\mathbf{1}}$ ). From the geometric characterization of the gamut provided by Theorem 1, we can see (see Fig. 5) that each locus of constant chromaticity intersects the gamut $\mathcal{G}$ in a line segment whose two end points are $\mathbf{t}_{\mathbf{0}}$ and a tristimulus $\overline{\mathbf{t}}$ located on a facet with an origin different from $\mathbf{t}_{\mathbf{0}}$. A consequence of this observation is that the desired property of linearity along lines of constant chromaticity completely specifies a $\mathrm{CCF}$, which we called the axially linear CCF $\overline{\mathscr{C}}$ in [33]. We now highlight how the facet description of the gamut facets and the uniqueness of the control values on the gamut surface can also be exploited conveniently for the construction of the axially linear CCF and present this construction using the notation and framework introduced in this paper. Throughout this section we assume that $\mathbf{t}_{\mathbf{0}}=\mathbf{0}$.

In tristimulus space, loci of constant chromaticity are rays, i.e., sets of the type $\{\gamma \tilde{\mathbf{t}} \mid \gamma \geq 0\}$ for a nonzero tristimulus $\tilde{\mathbf{t}}$ with nonnegative real-valued entries. Using the gamut representation from Theorem 1, as illustrated in Fig. 5, we can see that such a ray intersects the gamut $\mathcal{G}$ in a line segment whose two end points are $\mathbf{t}_{0}$ and a tristimulus $\overline{\mathbf{t}}$ located on a facet with an origin different from $\mathbf{t}_{\mathbf{0}}$. Consider a CCF $\overline{\mathscr{C}}(\cdot)$ that is linear along this line segment (See Fig. 5). The uniqueness of control vectors for points on the boundary defines $\overline{\mathscr{C}}$ at $\overline{\mathbf{t}}$ and $\mathbf{t}_{\mathbf{0}}$, and for any other tristimulus $\mathbf{t}_{\beta}=\beta \overline{\mathbf{t}}, 0<\beta<1$ located in the interior of the line segment of constant chromaticity, the of linearity $\overline{\mathscr{C}}$ implies that $\overline{\mathscr{C}}(\mathbf{t})=\beta \overline{\mathscr{C}}(\overline{\mathbf{t}})$. Now $\overline{\mathbf{t}}$ belongs to some gamut facet $\mathcal{F}\left(\mathbf{d}^{\mathbb{I} *}, \mathbf{P}[\mathbb{J}]\right)$ with origin $\mathbf{d}^{\mathbb{I} *}$ and spanned by the primaries $\mathbf{P}[\mathbb{J}]$, where $\mathbf{d}^{\mathbb{J} *}=\mathbf{t}_{0}+\mathbf{P} \chi^{\mathbb{J} *}=\mathbf{P} \chi^{\mathbb{J} *}$ and $\chi^{\mathbb{J} *} \in\left\{\chi^{\mathbb{J}-}, \chi^{\mathbb{J}+}\right\}$. It follows that $\overline{\mathbf{t}}=\mathbf{d}^{\mathbb{J} *}+\alpha_{\mathbb{J}[1]} \mathbf{p}_{\mathbb{J}[1]}+\alpha_{\mathbb{J}[2]} \mathbf{p}_{\mathbb{J}[2]}$, for some $\alpha_{\mathbb{J}[1]}, \alpha_{\mathbb{J}[2]} \in[0,1]$ and, therefore,

$$
\overline{\mathbf{t}}=\mathbf{P}\left(\boldsymbol{\chi}^{\mathbb{J} *}+\alpha_{\mathbb{J}[1]} \mathbf{e}_{\mathbb{J}[1]}^{K}+\alpha_{\mathbb{J}[2]} \mathbf{e}_{\mathbb{J}[2]}^{K}\right)=\mathbf{P A v}=\mathbf{M} \mathbf{v},
$$

where $\mathbf{A}=\left[\boldsymbol{\chi}^{\mathbb{J} *}, \mathbf{e}_{\mathbb{J}[1]}, \mathbf{e}_{\mathbb{J}[2]}\right]$ is a $K \times 3$ matrix, $\mathbf{v}=\left[1, \alpha_{\mathbb{J}[1]}, \alpha_{\mathbb{J}[2]}\right]^{T}$ is a $1 \times 3$ vector, and $\mathbf{M}=$ $\left[\mathbf{d}^{\mathbb{I} *}, \mathbf{p}_{\mathbb{J}[1]}, \mathbf{p}_{\mathbb{J}[2]}\right]=\mathbf{P A}$ is a $3 \times 3$ nonsingular matrix. From the uniqueness of control vectors on gamut the surface, it follows from (40) that $\overline{\mathscr{C}}(\overline{\mathbf{t}})=\mathbf{A v}$. Observing that $\mathbf{t}_{\beta}=\beta \mathbf{M v}$, we see that $\mathbf{v}=(1 / \beta) \mathbf{M}^{-1} \mathbf{t}_{\beta}$, therefore, the axially linear $\mathrm{CCF}$ at $\mathbf{t}_{\beta}$ is

$$
\overline{\mathscr{C}}\left(\mathbf{t}_{\beta}\right)=\beta \overline{\mathscr{C}}(\overline{\mathbf{t}})=\beta \mathbf{A} \mathbf{v}=\mathbf{A} \mathbf{M}^{-1} \mathbf{t}_{\beta} .
$$

Now, observing that the facet $\mathcal{F}\left(\mathbf{d}^{\mathbb{I} *}, \mathbf{P}[\mathbb{J}]\right)$ is the base of a quadrangle pyramid $\mathcal{Q}$ with apex $\mathbf{t}_{0}$, as shown in Fig. 5, we see that the expression in (41) applies for the CCF $\overline{\mathscr{C}}(\cdot)$ throughout the pyramid $\mathcal{Q}$. By considering the remaining facets not containing $\mathbf{t}_{0}$, we see that the axially linear $\operatorname{CCF} \overline{\mathscr{C}}(\cdot)$ is piecewise linear function of $\mathbf{t}$, and (41) induces a partition of the gamut $\mathcal{G}$ into quadrangle pyramids over each of which the $\overline{\mathscr{C}}(\cdot)$ is linear and expressed in the form of (41). Thus the

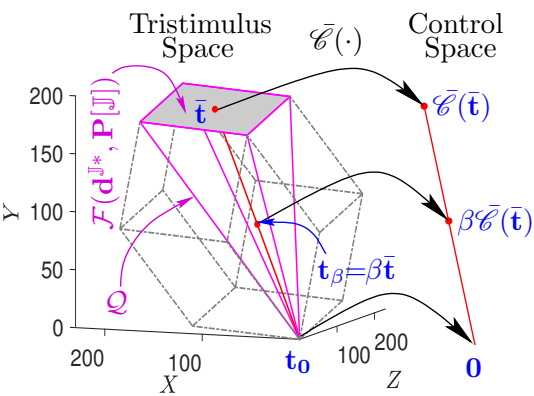

Fig. 5. Obtaining the axially linear $\mathrm{CCF} \overline{\mathscr{C}}$ from the geometric representation of the gamut provided by Theorem 1 .

requirement of linearity along gamut line segments of constant chromaticity, completely specifies the axially linear CCF $\overline{\mathscr{C}}(\cdot)$ over the entire gamut. We note that the quadrangle pyramid partitioning is also obtained, following a different motivation and approach, by the Matrix Switching methodology [34], from which an equivalent $\mathrm{CCF}$ is specified as a set of matrices, one for each quadrangle pyramid. Note that the number of pyramids varies from primary system to primary system. Because of convexity and the linear independence constraints on the primaries, every gamut contains at least 3 and at most $K$ facets containing the origin, thus the number of quadrangular pyramids partitioning the gamut lies between $K(K-1)-3$ and $K(K-1)-K$. Our methodology developed using Theorem 1 and Algorithm 1 is, however, general and applicable in all cases. By way of illustration, we present two examples of four primary systems with different numbers of quadrangular pyramids. Figure 6 illustrates the gamut partitioning for the four primary system $\mathbf{P}$ defined in (35), where there are 8 quadrangle pyramids (out of the 12 gamut facets, 4 contain the origin $\mathbf{t}_{0}=\mathbf{0}$ ). Fig. 7 illustrates the pyramid decomposition into the 9 quadrangle pyramids for the gamut of the $K=4$ primary system $\mathbf{P}_{w}^{(4)}$.

\section{CONCLUSION AND DISCUSSION}

The mathematical results presented in this paper provide a complete characterization of the geometry of multiprimary display gamuts. Based on Theorem 1 and the associated Algorithm 1, a geometric representation of the gamut can be readily computed, which can then be effectively exploited in color imaging applications. Examples of such applications were briefly outlined in Section V. Specifically, the parallelepiped tiling representation provided in Clause 1 of Theorem 1 is useful for computing gamut volumes in tristimulus and perceptual color spaces, which is useful as a figure of merit for the design of primaries for multiprimary displays and was our original motivation for exploring the gamut geometry [7]. Via Clauses 2-5, Theorem 1 also provides a complete characterization of the surface of the gamut, which is useful for assessing which colors are included within the gamut, for gamut mapping, and for designing color control strategies with desirable attributes. Theorem 1 also partly characterizes the flexibility of color control that is afforded by multiprimary displays. Specifically, Clause 6 asserts the uniqueness of control values for colors on the gamut surface, 


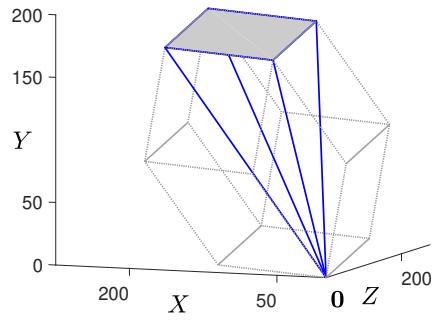

(a)

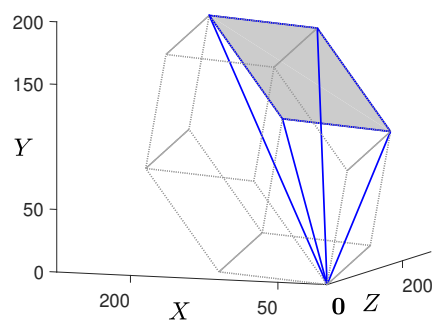

(c)

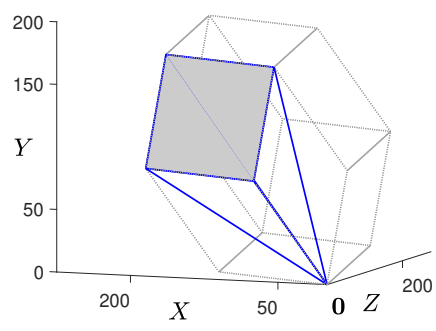

(e)

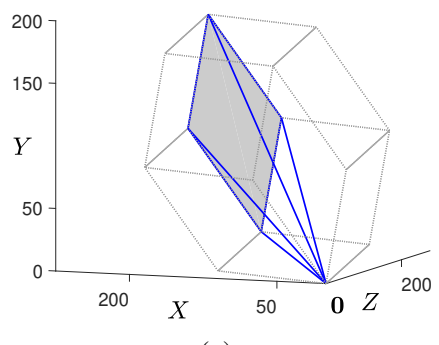

(g)

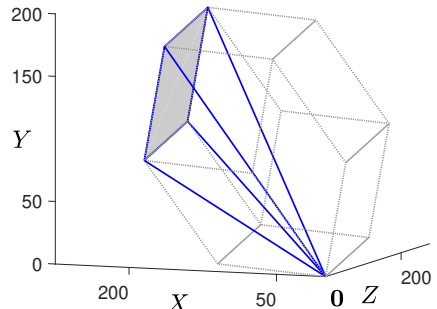

(b)

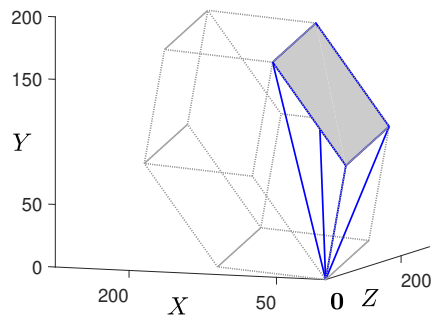

(d)

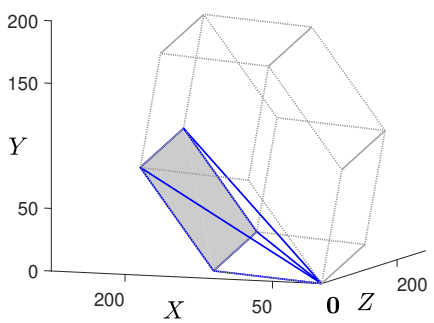

(f)

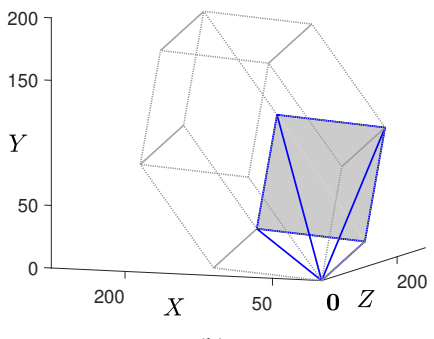

(h)
Fig. 6. The decomposition of the tristimulus gamut of the four primary system $\mathbf{P}$ into eight quadrangle pyramids for the axially linear CCF.

which was also previously shown in [11]. The different CCFs obtained by using Clause 7 in combination with alternative orderings of the primaries, also start to characterize color control options for colors in the interior of the gamut, which we further develop and fully characterize in the companion Part II paper [12]. Taken together these two papers provide a complete mathematical characterization of the geometry of the color gamut and control options for multiprimary displays.

As noted earlier, the display gamut belongs to the family of convex polytopes called zonotopes, whose definition as a Minkowski sum of set of line segments [10, Chap. 7] is a generalization of (4), wherein $\mathbf{P}$ is a general $d \times K$ matrix instead the more constrained setting for our multiprimary display gamuts, where we assumed that $\mathbf{P}$ is a $3 \times K$ matrix with nonnegative entries for which any three columns are linearly

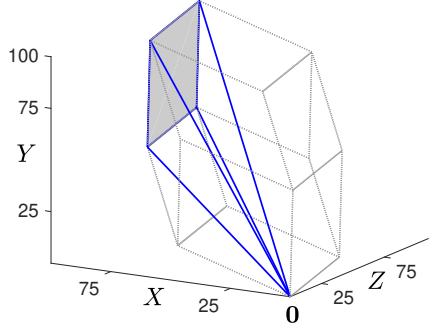

(a)

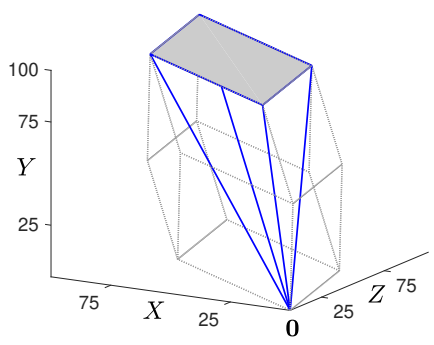

(c)

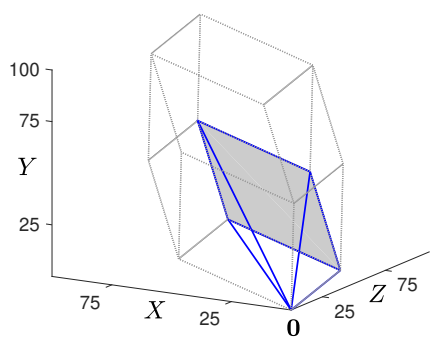

(e)

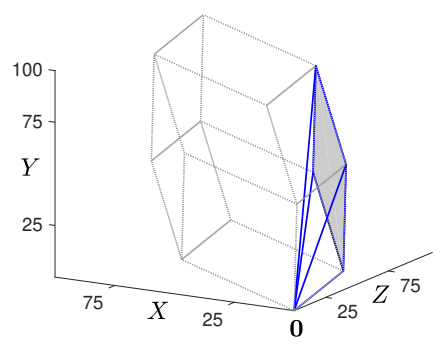

(g)

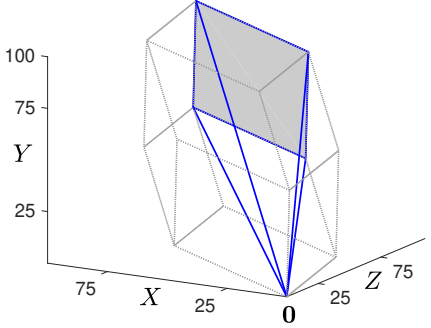

(b)

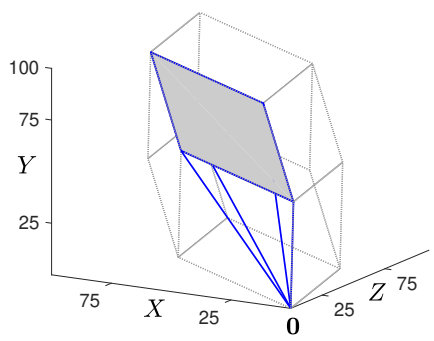

(d)

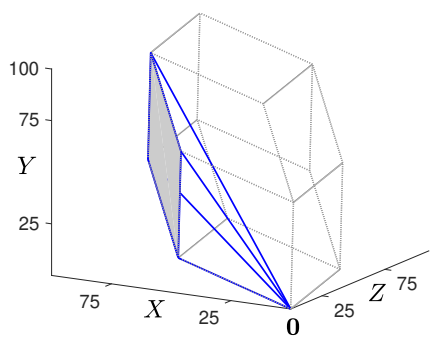

(f)

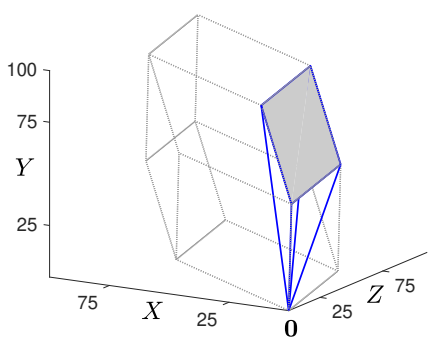

(h)

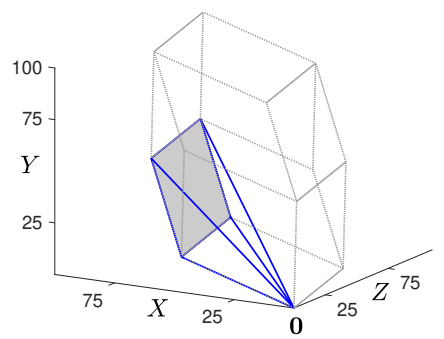

(i)

Fig. 7. The decomposition of the tristimulus gamut of the four primary system $\mathbf{P}_{w}^{(4)}$ into nine quadrangle pyramids for the axially linear CCF. 
independent. Our main mathematical results in Theorem 1 are obtained in the setting of $d=3$ dimensional zonotopes. Clauses of the theorem, other than Clause 5, are applicable, more generally, for any three dimensional zonotope, where $\mathbf{P}$ is a $3 \times K$ matrix for which any three columns are linearly independent. The physically motivated constraint of the nonnegative entries in $\mathbf{P}$ is sufficient though not necessary for Clause 5 to apply. Our proof of Theorem 1 demonstrates that Clause 5 also applies more broadly for $d=3$ dimensional zonotopes where $\mathbf{P}$ satisfies the constraint that no column of $\mathbf{P}$ can be formed as a linear combination of three other columns using exclusively negative coefficients ${ }^{12}$. The theorem therefore also provides a direct way to obtain geometric representations of more general $d=3$ dimensional zonotopes beyond multiprimary gamuts. Specifically, we note that polar zonohedra, which are three-dimensional zonotopes that have received particular attention in prior mathematical explorations [35], satisfy the constraint that no column of $\mathbf{P}$ can be formed as a linear combination of three other columns using exclusively negative coefficients and Theorem 1 therefore applies in its entirety to polar zonohedra. Appendix $G$ provides examples of geometric representations of three-dimensional polar zonohedra obtained using Algorithm 1 and Theorem 1. We note that our problem setting of multiprimary displays also motivated new mathematical questions that have not arisen in prior mathematical studies of zonotopes as abstract mathematical geometric objects. Specifically, the tiling CCF in Clause 7 of Theorem 1 arises naturally in the multiprimary display context due to our interest in color control, whereas such functions have not previously been studied in the context of general zonotopes. Generalizations of these tiling CCFs, which we address in the companion Part II paper [12], specifically provide a complete characterization of the flexibility in color control afforded by multiprimary displays. Finally, we note that from a mathematical perspective, extensions of our results to $d>3$ dimensional zonotopes would also be of interest, though these are clearly beyond the scope of the present work.

\section{APPENDIX A}

\section{PROOF OF LEMMA 1}

From the non-singularity of $\mathbf{A}$ it follows that the set $\mathcal{P}(\mathbf{v}, \mathbf{A})$ has the alternative equivalent representation

$$
\mathcal{P}(\mathbf{v}, \mathbf{A})=\left\{\mathbf{t} \in \mathbb{R}^{3} \mid \mathbf{0} \leq \mathbf{A}^{-1}(\mathbf{t}-\mathbf{v}) \leq \mathbf{1}\right\},
$$

where $\mathbf{0}$ and $\mathbf{1}$ denote the $3 \times 1$ vectors of zeros and ones, respectively, and the inequalities relating vectors apply termwise. From Cramer's rule [36, pp. 200] we can write

$$
\begin{gathered}
\mathbf{A}^{-1}=\frac{1}{\operatorname{det}(\mathbf{A})}\left[\begin{array}{r}
(\otimes \mathbf{A}[23])^{T} \\
-(\otimes \mathbf{A}[13])^{T} \\
(\otimes \mathbf{A}[12])^{T}
\end{array}\right], \\
\operatorname{det}(\mathbf{A})=(-1)^{(i+1)}(\otimes \mathbf{A}[\mathbb{J}])^{T} \mathbf{a}_{i}, \quad \mathbb{J} \in \mathfrak{C}^{2}(\langle 3\rangle),
\end{gathered}
$$

\footnotetext{
${ }^{12}$ Appendix $\mathrm{F}$ provides an example of a zonotope in $\mathbb{R}^{3}$ for which Clause 5 of Theorem 1 does not apply.
}

where $i=\langle 3\rangle \backslash \mathbb{J}$, and $\operatorname{det}(\cdot)$ denotes the determinant. It follows that the $i^{\text {th }}$ row of $\mathbf{A}^{-1}$ is

$$
\mathbf{n}_{\mathbb{J}}=\frac{1}{(\otimes \mathbf{A}[\mathbb{J}])^{T} \mathbf{a}_{i}}(\otimes \mathbf{A}[\mathbb{J}])=\operatorname{sgn}\left(\mathbf{u}_{\mathbb{J}}^{T} \mathbf{a}_{i}\right)\left\|\mathbf{n}_{\mathbb{J}}\right\| \mathbf{u}_{\mathbb{J}},
$$

where $\mathbb{J}=\langle 3\rangle \backslash i$. Using these expressions, through arithmetic manipulation (42) can be rewritten as

$$
\begin{aligned}
\mathcal{P}(\mathbf{v}, \mathbf{A}) & =\bigcap_{\mathbb{J} \in \mathfrak{C}^{2}(\langle 3\rangle)}\left\{\mathbf{t} \in \mathbb{R}^{3} \mid \tau^{\mathbb{J}} \leq \mathbf{u}_{\mathbb{J}}^{T} \mathbf{t} \leq \nu^{\mathbb{J}}\right\} \\
& =\bigcap_{\mathbb{J} \in \mathfrak{C}^{2}(\langle 3\rangle)} \mathcal{H}\left(\mathbf{u}_{\mathbb{J}}, \tau^{\mathbb{J}}, \nu^{\mathbb{J}}\right) .
\end{aligned}
$$

Note that $\nu^{\mathbb{J}}>\tau^{\mathbb{J}}$, so the interval $\left[\tau^{\mathbb{J}}, \nu^{\mathbb{J}}\right]$ is non-empty. As an affine map of the (bounded) unit cube, $\mathcal{P}(\mathbf{v}, \mathbf{A})$ is readily seen to be bounded and is therefore a polytope.

It is readily seen that for the unit cube $[0,1]^{3}=\mathcal{P}\left(\mathbf{0}, \mathbf{I}_{3}\right)$, the interior, surface, and vertices (extremal points) are, respectively, given by: (a) $(0,1)^{3}$, (b) points $\boldsymbol{\alpha}=\left[\alpha_{1}, \alpha_{2}, \alpha_{3}\right]^{T}$ where $\alpha_{l} \in\{0,1\}$ for some $0 \leq l \leq 3$, and (c) points $\alpha=$ $\left[\alpha_{1}, \alpha_{2}, \alpha_{3}\right]^{T}$ where $\alpha_{l} \in\{0,1\}$ for all $0 \leq l \leq 3$. Using our established notation, the unit cube and its faces can be compactly expressed as the sets $\mathcal{S}\left(\sum_{l \in\langle 3\rangle \backslash \mathbb{I}} \delta_{l} \mathbf{e}_{l}^{3}, \mathbf{I}_{3}[\mathbb{I}]\right)$, where $\mathbb{I} \subset$ $\langle 3\rangle, \delta_{l} \in\{0,1\}$, and $\mathscr{N}(\mathbb{I})$ is the dimensionality of the face. Using this expression, for $0 \leq l \leq 2$, the number of $l$-faces of the cube is seen to be $2^{3-l}\left(\begin{array}{l}3 \\ l\end{array}\right)$. Specifically, for $\mathscr{N}(\mathbb{I})=3$, we obtain the cube $[0,1]^{3}=\mathcal{S}\left(\mathbf{0}, \mathbf{I}_{3}\right)$; for $\mathscr{N}(\mathbb{I})=2$ we obtain the six facets $\mathcal{F}\left(\delta_{l} \mathbf{e}_{l}^{3}, \mathbf{I}_{3}[\mathbb{I}]\right), \mathbb{I} \in \mathfrak{C}^{2}(\langle 3\rangle), l=\langle 3\rangle \backslash \mathbb{I}$; for $\mathscr{N}(\mathbb{I})=$ 1 we obtain the twelve edges $\mathcal{E}\left(\sum_{l \in\langle 3\rangle \backslash i} \delta_{l} \mathbf{e}_{l}^{3}, \mathbf{e}_{i}^{3}\right), i \in\langle 3\rangle$; and for $\mathscr{N}(\mathbb{I})=0$ we obtain the eight vertices $\sum_{l=1}^{3} \delta_{l} \mathbf{e}_{l}^{3}$, where the $\delta_{l}$ terms are chosen from $\{0,1\}$ in all cases. An alternative expression for the facets is obtained by noting that for each $\mathbb{I} \in \mathfrak{C}^{2}(\langle 3\rangle)$, setting $l=\langle 3\rangle \backslash \mathbb{I}$ and $\xi=\left(\mathbf{I}_{3}[\mathbb{I}]^{T} \mathbf{I}_{3}[l]\right) \in\{-1,1\}$, we can write $\mathcal{F}\left(\delta_{l} \mathbf{e}_{l}^{3}, \mathbf{I}_{3}[\mathbb{I}]\right)=$ $\overline{\mathcal{H}}\left(\otimes \mathbf{I}_{3}[\mathbb{I}], \xi \delta_{l}\right) \bigcap\left(\bigcap_{\mathbb{I}^{\prime} \in \mathfrak{C}^{2}(\langle 3\rangle) \backslash \mathbb{I}} \mathcal{H}\left(\xi^{\prime} \otimes \mathbf{I}_{3}\left[\mathbb{I}^{\prime}\right], 0,1\right)\right)$, where for $\mathbb{I}^{\prime} \in \mathfrak{C}^{2}(\langle 3\rangle) \backslash \mathbb{I}, \xi^{\prime}=\left(\mathbf{I}_{3}\left[\mathbb{I}^{\prime}\right]^{T} \mathbf{I}_{3}\left[\langle 3\rangle \backslash \mathbb{I}^{\prime}\right]\right) \in\{-1,1\}$. The facets represent an essentially disjoint partitioning of the surface of the cube. Note also that each facet contains four edges, and each edge contains two vertices.

Since the parallelepiped $\mathcal{P}(\mathbf{v}, \mathbf{A})$ is a non-singular affine mapping of the unit cube $[0,1]^{3}=\mathcal{P}\left(\mathbf{0}, \mathbf{I}_{3}\right)$, the interior of the cube is mapped to the interior of the parallelepiped, while the surface and extremal points of the cube are mapped to the surface and extremal points of $\mathcal{P}(\mathbf{v}, \mathbf{A})$. The parallelepiped and the faces are therefore enumerated as $\mathcal{S}\left(\mathbf{v}+\mathbf{A} \sum_{l \in\langle 3\rangle \backslash \mathbb{I}} \delta_{l} \mathbf{e}_{l}^{3}, \mathbf{A I}_{3}[\mathbb{I}]\right)=\mathcal{S}\left(\mathbf{v}+\sum_{l \in\langle 3\rangle \backslash \mathbb{I}} \delta_{l} \mathbf{a}_{l}, \mathbf{A}[\mathbb{I}]\right)$, with $\delta_{l} \in\{0,1\}$. Specifically, the six facets are $\mathcal{F}(\mathbf{v}+$ $\left.\sum_{l \in\langle 3\rangle \backslash \mathbb{I}} \delta_{l} \mathbf{a}_{l}, \mathbf{A}[\mathbb{I}]\right), \mathbb{I} \in \mathfrak{C}^{2}(\langle 3\rangle)$; the twelve edges are $\mathcal{E}\left(\mathbf{v}+\sum_{l \in\langle 3\rangle \backslash i} \delta_{l} \mathbf{a}_{l}, \mathbf{a}_{i}\right), i \in\langle 3\rangle$; and the eight vertices are $\mathbf{v}+\sum_{l=1}^{3} \delta_{l} \mathbf{a}_{l}$. The surface of the parallelepiped is obtained as the essentially disjoint union of its distinct facets

$$
\begin{aligned}
\mathcal{B}_{\mathcal{P}(\mathbf{v}, \mathbf{A})} & =\bigcup_{\mathbb{J} \in \mathfrak{C}^{2}(\langle 3\rangle)}\left(\mathcal{F}(\mathbf{v}, \mathbf{A}[\mathbb{J}]) \cup \mathcal{F}\left(\mathbf{v}+\mathbf{a}_{i}, \mathbf{A}[\mathbb{J}]\right)\right) \\
& =\bigcup_{\mathbb{J} \in \mathfrak{C}^{2}(\langle 3\rangle)}\left(\mathcal{F}\left(\mathbf{d}^{\mathbb{J}^{-}}, \mathbf{A}[\mathbb{J}]\right) \cup \mathcal{F}\left(\mathbf{d}^{\mathbb{J}^{+}}, \mathbf{A}[\mathbb{J}]\right)\right)
\end{aligned}
$$


where the last step is obtained by noting that whenever $\chi^{-}\left(\mathbf{u}_{\mathfrak{J}}^{T} \mathbf{a}_{i}\right)=1$, then $\chi^{+}\left(\mathbf{u}_{\mathfrak{J}}^{T} \mathbf{a}_{i}\right)=0$ and vice versa, so that $\mathbf{d}^{\mathbb{J}^{-}}$and $\mathbf{d}^{\mathbb{J}^{+}}$represent the vectors $\mathbf{v}$ and $\mathbf{v}+\mathbf{a}_{i}$ (possibly in a different order). Observe that the origin for any face does not include any of the primaries that span the face.

Note that $\mathbf{t} \in \mathcal{P}(\mathbf{v}, \mathbf{A}) \bigcap \overline{\mathcal{H}}\left(\mathbf{u}_{\mathbb{J}}, \tau^{\mathbb{J}}\right) \Leftrightarrow \mathbf{u}_{\mathbb{J}}^{T} \mathbf{t}=\tau^{\mathbb{J}}$ and for some $\boldsymbol{\alpha} \in[0,1]^{3}, \mathbf{t}=\mathbf{A} \boldsymbol{\alpha}+\mathbf{v}=\mathbf{A}[\mathbb{J}] \boldsymbol{\alpha}[\mathbb{J}]+\alpha_{i} \mathbf{a}_{i}+\mathbf{v}$, with $i=\langle 3\rangle \backslash \mathbb{J} \Leftrightarrow \mathbf{u}_{\mathbb{J}}^{T}\left(\alpha_{i} \mathbf{a}_{i}+\mathbf{v}\right)=\tau^{\mathbb{J}}=\mathbf{u}_{\mathbb{J}}^{T}\left(\chi^{-}\left(\mathbf{u}_{\mathbb{J}}^{T} \mathbf{a}_{i}\right) \mathbf{a}_{i}+\mathbf{v}\right)$ $\Leftrightarrow \alpha_{i}=\chi^{-}\left(\mathbf{u}_{\mathbb{J}}^{T} \mathbf{a}_{i}\right) \Leftrightarrow \mathbf{t}=\mathbf{A}[\mathbb{J}] \boldsymbol{\alpha}[\mathbb{J}]+\mathbf{d}^{\mathbb{J}^{-}} \in \mathcal{F}\left(\mathbf{d}^{\mathbb{J}^{-}}, \mathbf{A}[\mathbb{J}]\right)$. Therefore $\mathcal{F}\left(\mathbf{d}^{\mathbb{I}^{-}}, \mathbf{A}[\mathbb{J}]\right)=\mathcal{P}(\mathbf{v}, \mathbf{A}) \cap \overline{\mathcal{H}}\left(\mathbf{u}_{\mathbb{J}}, \tau^{\mathbb{J}}\right)$. From the alternative expression for the facets of the $[0,1]^{3}$ cube presented above, we can also see that $\mathcal{F}\left(\mathbf{d}^{\mathbb{J}^{-}}, \mathbf{A}[\mathbb{J}]\right)=$ $\overline{\mathcal{H}}\left(\mathbf{u}_{\mathbb{J}}, \tau^{\mathbb{J}}\right) \cap\left(\bigcap_{\mathbb{J}^{\prime} \in \mathfrak{C}^{2}(\langle 3\rangle) \backslash \mathbb{J}} \mathcal{H}\left(\mathbf{u}_{\mathbb{J}^{\prime}}, \tau^{\mathbb{J}^{\prime}}, \nu^{\mathbb{J}^{\prime}}\right)\right)$ and that the planes $\overline{\mathcal{H}}\left(\mathbf{u}_{\mathbb{J}^{\prime}}, \tau^{\mathbb{J}^{\prime}}\right)$ and $\overline{\mathcal{H}}\left(\mathbf{u}_{\mathbb{J}^{\prime}}, \nu^{\mathbb{J}^{\prime}}\right)$ for $\mathbb{J}^{\prime} \in \mathfrak{C}^{2}(\langle 3\rangle) \backslash \mathbb{J}$ support the edges of the facet $\mathcal{F}\left(\mathbf{d}^{\mathbb{J}^{-}}, \mathbf{A}[\mathbb{J}]\right)$. Similarly, we conclude that $\mathcal{F}\left(\mathbf{d}^{\mathbb{J}^{+}}, \mathbf{A}[\mathbb{J}]\right)=\mathcal{P}(\mathbf{v}, \mathbf{A}) \bigcap \overline{\mathcal{H}}\left(\mathbf{u}_{\mathbb{J}}, \nu^{\mathbb{J}}\right)=$ $\overline{\mathcal{H}}\left(\mathbf{u}_{\mathbb{J}}, \nu^{\mathbb{J}}\right) \cap\left(\bigcap_{\mathbb{J}^{\prime} \in \mathfrak{C}^{2}(\langle 3\rangle) \backslash \mathbb{J}} \mathcal{H}\left(\mathbf{u}_{\mathbb{J}^{\prime}}, \tau^{\mathbb{J}^{\prime}}, \nu^{\mathbb{J}^{\prime}}\right)\right)$, where the edges of these facets are supported by the planes $\overline{\mathcal{H}}\left(\mathbf{u}_{\mathbb{J}^{\prime}}, \tau^{\mathbb{J}^{\prime}}\right)$ and $\overline{\mathcal{H}}\left(\mathbf{u}_{\mathbb{J}^{\prime}}, \nu^{\mathbb{J}^{\prime}}\right)$ for $\mathbb{J}^{\prime} \in \mathfrak{C}^{2}(\langle 3\rangle) \backslash \mathbb{J}$. Next we note that the non-singular affine mappings to/from $\mathcal{P}\left(\mathbf{0}, \mathbf{I}_{3}\right)=[0,1]^{3}$ place the edges and vertices in $\mathcal{P}(\mathbf{v}, \mathbf{A})$ in one-to-one correspondence with the edges and vertices in $\mathcal{P}\left(\mathbf{0}, \mathbf{I}_{3}\right)$, respectively, and infact with every other parallelepiped $\mathcal{P}\left(\mathbf{v}^{\prime}, \mathbf{A}^{\prime}\right)$ where $\mathbf{v}^{\prime} \in \mathbb{R}^{3}$ and $\mathbf{A}^{\prime} \in \mathbb{R}^{3 \times 3}$ is non-singular. The fact that Clauses 3 and 4 of Lemma 1 enumerate the edges and vertices exhaustively and uniquely is then readily seen by verifying it for any single example (See Fig. 1).

Finally, the non-singularity of A directly implies that the mapping $\boldsymbol{\alpha} \mapsto \mathbf{A} \boldsymbol{\alpha}+\mathbf{v}$ and its inverse mapping $\mathbf{t} \mapsto$ $\mathbf{A}^{-1}(\mathbf{t}-\mathbf{v}) \stackrel{\text { def }}{=} \boldsymbol{\beta}(\mathbf{t})$ are bijective linear (and continuous) functions from $\mathbb{R}^{3}$ onto $\mathbb{R}^{3}$. From the fact that $\mathcal{P}(\mathbf{v}, \mathbf{A})$ is the range of $[0,1]^{3}$ under the map $\boldsymbol{\alpha} \mapsto \mathbf{A} \boldsymbol{\alpha}+\mathbf{v}$, it directly follows that for any $\mathbf{t} \in \mathcal{P}(\mathbf{v}, \mathbf{A}), \boldsymbol{\alpha}=\mathbf{A}^{-1}(\mathbf{t}-\mathbf{v}) \in[0,1]^{3}$ is the unique feasible control vector such that $\mathbf{t}=\mathbf{A} \boldsymbol{\alpha}+\mathbf{v}$. From (43) we also directly see that, for $i=1,2,3, \beta_{i}(\mathbf{t})=$ $(-1)^{(i+1)}(\otimes \mathbf{A}[\mathbb{J}])^{T}(\mathbf{t}-\mathbf{v}) / \operatorname{det}(\mathbf{A})$, where $\mathbb{J}=\langle 3\rangle \backslash i$.

\section{APPENDIX B \\ PROOF OF THEOREM 1}

Prior to proving the theorem, we establish a couple of useful sub-results for our proof as the following lemmas.

Lemma 2. For a $K \geq 4$ primary display, control values are non-unique for any tristimulus lying in the interior of the gamut $\mathcal{G}$.

Proof. Suppose $\mathbf{t}=\mathbf{t}_{\mathbf{0}}+\mathbf{P} \boldsymbol{\alpha}$ lies in the interior of the gamut $\mathcal{G}$. There exists a positive real number $\epsilon$ such that the ball $\mathcal{D}(\mathbf{t}, \epsilon)$ of radius $\epsilon$ centered at $\mathbf{t}$ is contained in $\mathcal{G}$. Since the map $\boldsymbol{\alpha}^{\prime}:[0,1]^{K} \rightarrow \mathcal{G}$, defined as $\boldsymbol{\alpha}^{\prime} \mapsto \mathbf{t}_{\mathbf{0}}+\mathbf{P} \boldsymbol{\alpha}^{\prime}$ is continuous and $\mathcal{D}(\mathbf{t}, \epsilon)$ is an open set relative to $\mathcal{G}$, the inverse image of $\mathcal{D}(\mathbf{t}, \epsilon)$ is an open set $\Psi \subset[0,1]^{K}$. Clearly $\boldsymbol{\alpha} \in \Psi$ so there exists a positive real number $\delta$ such that the ball
$\mathcal{D}(\boldsymbol{\alpha}, \delta)$ of radius $\delta$ centered at $\boldsymbol{\alpha}$ is contained in $\Psi$. Now, because $K>3$, there exists a nonzero vector $\zeta$ in the null space of $\mathbf{P}$, so for some positive real number $\delta_{2}$, the vector $\gamma=\boldsymbol{\alpha}+\delta_{2} \boldsymbol{\zeta} \in \mathcal{D}(\boldsymbol{\alpha}, \delta) \subset \Psi$. Observing that $\boldsymbol{\alpha} \neq \gamma$ and $\mathbf{t}_{\mathbf{0}}+\mathbf{P} \gamma=\mathbf{t}_{\mathbf{0}}+\mathbf{P} \boldsymbol{\alpha}=\mathbf{t}$, we see that $\boldsymbol{\alpha}$ and $\gamma$ are two distinct control vectors for $\mathbf{t}$.

Lemma 3. If a tristimulus $\mathbf{t} \in \mathcal{F}\left(\mathbf{d}^{\mathbb{J}^{-}}, \mathbf{P}[\mathbb{J}]\right) \cup \mathcal{F}\left(\mathbf{d}^{\mathbb{J}^{+}}, \mathbf{P}[\mathbb{J}]\right)$, for some $\mathbb{J} \in \mathfrak{C}^{2}(\langle M\rangle)$, then the control vector for $\mathbf{t}$ is unique.

Proof. Assume that $\mathbf{t} \in \mathcal{F}\left(\mathbf{d}^{\mathbb{J}^{-}}, \mathbf{P}[\mathbb{J}]\right)$ for some $\mathbb{J} \in$ $\mathfrak{C}^{2}(\langle K\rangle)$ (the case for $\mathbf{t} \in \mathcal{F}\left(\mathbf{d}^{\mathbb{J}^{+}}, \mathbf{P}[\mathbb{J}]\right)$ follows analogous arguments). Now, let $\boldsymbol{\alpha}_{1}, \boldsymbol{\alpha}_{2} \in[0,1]^{K}$ be any two control vectors for $\mathbf{t}$. We will establish uniqueness by showing that $\boldsymbol{\alpha}_{1}=\boldsymbol{\alpha}_{2}$. For any $\boldsymbol{\alpha} \in[0,1]^{K}$, observing that we can write $\mathbf{u}_{\mathfrak{J}}^{T}\left(\mathbf{t}_{\mathbf{0}}+\mathbf{P} \boldsymbol{\alpha}\right)-\tau^{\mathbb{J}}=\sum_{k=1}^{K}\left|\left(\alpha_{k}-\chi^{-}\left(\mathbf{u}_{\mathfrak{J}}^{T} \mathbf{p}_{k}\right)\right) \mathbf{u}_{\mathfrak{J}}^{T} \mathbf{p}_{k}\right|$, we can see that $\left\{\boldsymbol{\alpha} \in[0,1]^{K} \mid \mathbf{u}_{\mathbb{J}}^{T}\left(\mathbf{t}_{\mathbf{0}}+\mathbf{P} \boldsymbol{\alpha}\right)=\tau^{\mathbb{J}}\right\}=\left\{\boldsymbol{\chi}^{\mathbb{J}^{-}}+\right.$ $\left.\mathbf{I}_{K}[\mathbb{J}] \boldsymbol{\beta} \mid \boldsymbol{\beta} \in[0,1]^{2}\right\}$. Therefore there exist $\boldsymbol{\beta}_{1}, \boldsymbol{\beta}_{2} \in[0,1]^{2}$, such that $\boldsymbol{\alpha}_{1}=\chi^{\mathbb{J}^{-}}+\mathbf{I}_{K}[\mathbb{J}] \boldsymbol{\beta}_{1}$ and $\boldsymbol{\alpha}_{2}=\chi^{\mathbb{J}^{-}}+\mathbf{I}_{K}[\mathbb{J}] \boldsymbol{\beta}_{2}$. Now, using the fact that $\mathbf{t}=\mathbf{t}_{\mathbf{0}}+\mathbf{P} \boldsymbol{\alpha}_{1}=\mathbf{t}_{\mathbf{0}}+\mathbf{P} \boldsymbol{\alpha}_{2}$, we have $\mathbf{P}[\mathbb{J}]\left(\boldsymbol{\beta}_{1}-\boldsymbol{\beta}_{2}\right)=0$. Because any selection of two primaries from $\mathbf{P}$ is linearly independent, it follows that $\boldsymbol{\beta}_{1}=\boldsymbol{\beta}_{2}$, whereby $\boldsymbol{\alpha}_{1}=\boldsymbol{\alpha}_{2}$.

We prove Theorem 1 by induction on $K$. For $K=3$ the result follows immediately from Lemma 1 . Next, for a positive integer $M \geq 4$, we assume the result holds for all integers $K \leq(M-1)$ and show that it holds for $K=M$. To simplify notation, in this section, we use $\mathbf{P}$ to consistently represent the matrix of all the $M$ primaries and rely on the sub-selection via indices to represent systems with fewer primaries. Also, we denote by $\mathcal{G}_{l}, \mathcal{B}_{l}$, and $\mathscr{C}_{l}$ the gamut, surface, and CCF, respectively, for the $l$-primary system with the primary matrix $\mathbf{P}[\langle l\rangle]$. Furthermore, we denote

$$
\begin{aligned}
\mathbf{d}_{l}^{\mathbb{J}^{-}} & =\mathbf{t}_{\mathbf{0}}+\mathbf{P} \chi_{l}^{\mathbb{J}^{-}}, \\
\mathbf{d}_{l}^{\mathbb{J}^{+}} & =\mathbf{t}_{\mathbf{0}}+\mathbf{P} \chi_{l}^{\mathbb{J}^{+}}, \\
\tau_{l}^{\mathbb{J}} & =\mathbf{u}_{\mathbb{J}}^{T} \mathbf{d}_{l}^{\mathbb{J}^{-}}, \\
\nu_{l}^{\mathbb{J}} & =\mathbf{u}_{\mathbb{J}}^{T} \mathbf{d}_{l}^{\mathbb{J}^{+}},
\end{aligned}
$$

Observe that these definitions imply that for any given $\mathbb{J}, \tau_{l}^{\mathbb{J}}$ and $\nu_{l}^{\mathbb{J}}$, viewed as sequences indexed by $l$, are, respectively, non-increasing and non-decreasing. Furthermore, for $l \geq 4$ and $\mathbb{J} \in \mathfrak{C}^{2}(\langle l-1\rangle)$, we can see that

$$
\left[\tau_{l}^{\mathbb{J}}, \nu_{l}^{\mathbb{J}}\right]=\left[\tau_{l-1}^{\mathbb{J}}, \nu_{l-1}^{\mathbb{J}}\right] \bigcup\left[\kappa_{l}^{\mathbb{J}}, \eta_{l}^{\mathbb{J}}\right],
$$

where

$$
\begin{aligned}
\kappa_{l}^{\mathbb{J}} & =\chi^{-}\left(\mathbf{u}_{\mathbb{J}}^{T} \mathbf{p}_{l}\right) \tau_{l}^{\mathbb{J}}+\chi^{+}\left(\mathbf{u}_{\mathbb{J}}^{T} \mathbf{p}_{l}\right) \nu_{l-1}^{\mathbb{J}}, \\
\eta_{l}^{\mathbb{J}} & =\chi^{-}\left(\mathbf{u}_{\mathbb{J}}^{T} \mathbf{p}_{l}\right) \tau_{l-1}^{\mathbb{J}}+\chi^{+}\left(\mathbf{u}_{\mathbb{J}}^{T} \mathbf{p}_{l}\right) \nu_{l}^{\mathbb{J}} .
\end{aligned}
$$

Also note that: (a) $\tau_{l-1}^{\mathbb{J}}>\nu_{l-1}^{\mathbb{J}}$, (b) either $\kappa_{l}^{\mathbb{J}}=\nu_{l-1}^{\mathbb{J}}$ or $\eta_{l}^{\mathbb{J}}=\tau_{l-1}^{\mathbb{J}}$, and (c) $\eta_{l}^{\mathbb{J}}>\kappa_{l}^{\mathbb{J}}$. Thus (52) represents an essentially disjoint union of intervals with nonempty interiors.

Now, for each $j \in\langle M-1\rangle$, we define the function

$$
f_{j}(\mathbf{t}) \stackrel{\text { def }}{=}\left(\mathbf{u}_{[j, M]}^{T} \mathbf{t}\right)
$$


for all $\mathbf{t} \in \mathbb{R}^{3}$. For $\boldsymbol{\alpha} \in[0,1]^{M}$, we note that we can write

$$
\begin{aligned}
& \mathbf{u}_{[j, M]}^{T}\left(\mathbf{t}_{\mathbf{o}}+\mathbf{P} \boldsymbol{\alpha}\right)-\tau_{M}^{[j, M]}= \\
& \sum_{\substack{k=1 \\
k \neq j}}^{M-1}\left|\left(\alpha_{k}-\chi^{-}\left(\mathbf{u}_{[j, M]}^{T} \mathbf{p}_{k}\right)\right) \mathbf{u}_{[j, M]}^{T} \mathbf{p}_{k}\right|, \\
& \nu_{M}^{[j, M]}-\mathbf{u}_{[j, M]}^{T}\left(\mathbf{t}_{\mathbf{0}}+\mathbf{P} \boldsymbol{\alpha}\right)= \\
& \quad \sum_{\substack{k=1 \\
k \neq j}}^{M-1}\left|\left(\alpha_{k}-\chi^{+}\left(\mathbf{u}_{[j, M]}^{T} \mathbf{p}_{k}\right)\right) \mathbf{u}_{[j, M]}^{T} \mathbf{p}_{k}\right| .
\end{aligned}
$$

Thus the minima (maxima) for the function $f_{j}(\mathbf{t})$ over $\mathbf{t} \in \mathcal{G}_{M}$ is $\tau_{M}^{[j, M]}\left(\nu_{M}^{[j, M]}\right)$ and achieved precisely over the set $\left\{\mathbf{t}_{\mathbf{0}}+\mathbf{P} \chi_{M}^{[j, M]-}+\mathbf{P}[j, M] \boldsymbol{\beta} \mid \boldsymbol{\beta} \in[0,1]^{2}\right\}=$ $\mathcal{F}\left(\mathbf{d}_{M}^{\mathbb{J}^{-}}, \mathbf{P}[j, M]\right)\left(\left\{\mathbf{t}_{\mathbf{0}}+\mathbf{P} \chi_{M}^{[j, M]+}+\mathbf{P}[j, M] \boldsymbol{\beta} \mid \boldsymbol{\beta} \in\right.\right.$ $\left.[0,1]^{2}\right\}=\mathcal{F}\left(\mathbf{d}_{M}^{\mathbb{J}^{+}}, \mathbf{P}[j, M]\right)$ ). Noting that $\tau_{M}^{[j, M]}=\tau_{M-1}^{[j, M]}$, $\nu_{M}^{[j, M]}=\nu_{M-1}^{[j, M]}, \chi_{M}^{[j, M]-}=\chi_{M-1}^{[j, M]-}, \chi_{M}^{[j, M]+}=\chi_{M-1}^{[j, M]+}$ and that the upper limits in the summations in (56) and (57) are $(M-1)$, we also conclude from the above equations that the minima (maxima) for the function $f_{j}(\mathbf{t})$ over $\mathbf{t} \in$ $\mathcal{G}_{M-1}$ is $\tau_{M-1}^{[j, M]}\left(\nu_{M-1}^{[j, M]}\right)$ and achieved precisely over the set $\left\{\mathbf{t}_{\mathbf{0}}+\mathbf{P} \chi_{M-1}^{[j, M]-}+\beta \mathbf{p}_{j} \mid \beta \in[0,1]\right\}=\mathcal{E}\left(\mathbf{d}_{M}^{\mathbb{J}^{-}}, \mathbf{p}_{j}\right)$ $\left(\left\{\mathbf{t}_{\mathbf{o}}+\mathbf{P} \boldsymbol{\chi}_{M-1}^{[j, M]+}+\beta \mathbf{p}_{j} \mid \beta \in[0,1]\right\}=\mathcal{E}\left(\mathbf{d}_{M}^{\mathbb{J}^{+}}, \mathbf{p}_{j}\right)\right)$.

From the gamut definition in (4), we have

$$
\mathcal{G}_{l}=\left\{\mathbf{P}[\langle l\rangle] \boldsymbol{\alpha}+\mathbf{t}_{\mathbf{0}} \mid \boldsymbol{\alpha} \in[0,1]^{l}\right\},
$$

for $l=1,2, \ldots M$. From the definition, it follows that $\mathcal{G}_{M-1} \subset \mathcal{G}_{M}$.

Then, via the induction hypothesis, we have

$$
\begin{aligned}
\mathcal{G}_{M-1} & =\bigcup_{i=3}^{M-1}\left(\bigcup_{\mathbb{J} \in \mathfrak{C}^{2}(\langle i-1\rangle)} \mathcal{P}\left(\mathbf{c}_{i}^{\mathbb{J}}, \mathbf{P}[\mathbb{J}, i]\right)\right), \\
& =\bigcap_{\mathbb{J} \in \mathfrak{C}^{2}(\langle M-1\rangle)} \mathcal{H}\left(\mathbf{u}_{\mathbb{J}}, \tau_{M-1}^{\mathbb{J}}, \nu_{M-1}^{\mathbb{J}}\right),
\end{aligned}
$$

and the surface of $\mathcal{G}_{M-1}$ is given by

$$
\mathcal{B}_{\mathcal{G}_{M-1}}=\bigcup_{\mathbb{J} \in \mathfrak{C}^{2}(\langle M-1\rangle)}\left(\mathcal{F}\left(\mathbf{d}_{M-1}^{\mathbb{J}^{-}}, \mathbf{P}[\mathbb{J}]\right) \cup \mathcal{F}\left(\mathbf{d}_{M-1}^{\mathbb{J}^{+}}, \mathbf{P}[\mathbb{J}]\right)\right),
$$

with

$$
\begin{aligned}
& \mathcal{F}\left(\mathbf{d}_{M-1}^{\mathbb{J}^{-}}, \mathbf{P}[\mathbb{J}]\right)=\mathcal{G}_{M-1} \bigcap \overline{\mathcal{H}}\left(\mathbf{u}_{\mathbb{J}}, \tau_{M-1}^{\mathbb{J}}\right), \\
& \mathcal{F}\left(\mathbf{d}_{M-1}^{\mathbb{J}^{+}}, \mathbf{P}[\mathbb{J}]\right)=\mathcal{G}_{M-1} \bigcap \overline{\mathcal{H}}\left(\mathbf{u}_{\mathbb{J}}, \nu_{M-1}^{\mathbb{J}}\right) .
\end{aligned}
$$

representing a pair of congruent parallelogram facets with supporting planes $\overline{\mathcal{H}}\left(\mathbf{u}_{\mathbb{J}}, \tau_{M-1}^{\mathbb{J}}\right)$ and $\overline{\mathcal{H}}\left(\mathbf{u}_{\mathbb{J}}, \nu_{M-1}^{\mathbb{J}}\right)$, respectively, for each $\mathbb{J} \in \mathfrak{C}^{2}(\langle M-1\rangle)$. Furthermore, each edge of a facet spanned by a primary $\mathbf{p}_{j}$ is supported by a plane $\overline{\mathcal{H}}\left(\mathbf{u}_{\bar{J}}, \tau^{\overline{\mathbb{J}}}\right)$ or $\overline{\mathcal{H}}\left(\mathbf{u}_{\overline{\mathbb{J}}}, \nu^{\bar{J}}\right)$ for some $\overline{\mathbb{J}} \in \mathfrak{C}^{2}(\langle K\rangle)$ such that $j \in \overline{\bar{J}}$. Also, for points on each of the boundary $\mathcal{B}_{\mathcal{G}_{M-1}}$ control values are uniquely determined. Specifically, the control vectors corresponding to any of the origins of the faces are uniquely determined.
For subsequent elements in our proof, we will find it convenient to relabel the facets, their supporting planes, and corresponding halfspaces in the preceding equations as follows. For each $\mathbb{J} \in \mathfrak{C}^{2}(\langle M-1\rangle)$, we denote the two half-spaces in the intersection $\mathcal{H}\left(\mathbf{u}_{\mathbb{J}}, \tau_{M-1}^{\mathbb{J}}, \nu_{M-1}^{\mathbb{J}}\right)$ as $\mathcal{H}^{<}\left(\mathbf{u}_{\mathbb{J}}, \phi_{M-1}^{\mathbb{J}}\right)$ and $\mathcal{H}^{>}\left(\mathbf{u}_{\mathbb{J}}, \psi_{M-1}^{\mathbb{J}}\right)$ based on whether $\mathbf{p}_{M}$ points into or out of the halfspace. Specifically, if $\mathbf{u}_{\mathbb{J}}^{T} \mathbf{p}_{M}<0$, set $\mathcal{H}^{<}\left(\mathbf{u}_{\mathbb{J}}, \phi_{M-1}^{\mathbb{J}}\right)=\mathcal{H}^{-}\left(\mathbf{u}_{\mathbb{J}}, \nu_{M-1}^{\mathbb{J}}\right)$ and $\mathcal{H}^{>}\left(\mathbf{u}_{\mathbb{J}}, \psi_{M-1}^{\mathbb{J}}\right)=\mathcal{H}^{+}\left(\mathbf{u}_{\mathbb{J}}, \tau_{M-1}^{\mathbb{J}}\right)$, where $\phi_{M-1}^{\mathbb{J}}=$ $\nu_{M-1}^{\mathbb{J}}=\nu_{M}^{\mathbb{J}}, \psi_{M-1}^{\mathbb{J}}=\tau_{M-1}^{\mathbb{J}}=\eta_{M}^{\mathbb{J}}$ and $\kappa_{M}^{\mathbb{J}}=\tau_{M-1}^{\mathbb{J}}+$ $\mathbf{u}_{\sqrt{J}}^{T} \mathbf{p}_{M}=\tau_{M}^{\mathbb{J}}$ so that we also have $\mathcal{H}^{-}\left(\mathbf{u}_{\mathbb{J}}, \nu_{M}^{\mathbb{J}}\right)=$ $\mathcal{H}^{<}\left(\mathbf{u}_{\mathbb{J}}, \phi_{M-1}^{\mathbb{J}}\right)$ and $\mathcal{H}^{+}\left(\mathbf{u}_{\mathbb{J}}, \tau_{M}^{\mathbb{J}}\right)=\mathcal{H}^{>}\left(\mathbf{u}_{\mathbb{J}}, \kappa_{M}^{\mathbb{J}}\right)=$ $\mathbf{p}_{M}+\mathcal{H}^{>}\left(\mathbf{u}_{\mathbb{J}}, \psi_{M}^{\mathbb{J}}\right)$. Otherwise, if $\mathbf{u}_{\mathbb{J}}^{T} \mathbf{p}_{M}>0$, set $\mathcal{H}^{<}\left(\mathbf{u}_{\mathbb{J}}, \phi_{M-1}^{\mathbb{J}}\right)=\mathcal{H}^{+}\left(\mathbf{u}_{\mathbb{J}}, \tau_{M-1}^{\mathbb{J}}\right)$ and $\mathcal{H}^{>}\left(\mathbf{u}_{\mathbb{J}}, \psi_{M-1}^{\mathbb{J}}\right)=$ $\mathcal{H}^{-}\left(\mathbf{u}_{\mathbb{J}}, \nu_{M-1}^{\mathbb{J}}\right)$, where $\phi_{M-1}^{\mathbb{J}}=\tau_{M-1}^{\mathbb{J}}, \psi_{M-1}^{\mathbb{J}}=\nu_{M-1}^{\mathbb{J}}=$ $\kappa_{M}^{\mathbb{J}}$, and $\eta_{M}^{\mathbb{J}}=\nu_{M-1}^{\mathbb{J}}+\mathbf{u}_{\mathbb{J}}^{T} \mathbf{p}_{M}=\nu_{M}^{\mathbb{J}}$ so that we also have $\mathcal{H}^{-}\left(\mathbf{u}_{\mathbb{J}}, \nu_{M}^{\mathbb{J}}\right)=\mathcal{H}^{>}\left(\mathbf{u}_{\mathbb{J}}, \eta_{M}^{\mathbb{J}}\right)=\mathbf{p}_{M}+\mathcal{H}^{>}\left(\mathbf{u}_{\mathbb{J}}, \psi_{M-1}^{\mathbb{J}}\right)$ and $\mathcal{H}^{+}\left(\mathbf{u}_{\mathbb{J}}, \tau_{M}^{\mathbb{J}}\right)=\mathcal{H}^{<}\left(\mathbf{u}_{\mathbb{J}}, \phi_{M-1}^{\mathbb{J}}\right)$. Note that in both cases, the halfspaces $\mathcal{H}^{<}\left(\mathbf{u}_{\mathbb{J}}, \phi_{M-1}^{\mathbb{J}}\right)$ and $\mathbf{p}_{M}+\mathcal{H}^{>}\left(\mathbf{u}_{\mathbb{I}}, \psi_{M-1}^{\mathbb{J}}\right)$ provide a relabeling of the halfspaces associated with the plane slice $\mathcal{H}\left(\mathbf{u}_{\mathbb{J}}, \tau_{M}^{\mathbb{J}}, \nu_{M}^{\mathbb{J}}\right)$ and therefore the planes $\overline{\mathcal{H}}\left(\mathbf{u}_{\mathbb{J}}, \phi_{M-1}^{\mathbb{J}}\right)$ and $\mathbf{p}_{M}+\overline{\mathcal{H}}\left(\mathbf{u}_{\mathbb{J}}, \psi_{M-1}^{\mathbb{J}}\right)$ also provide a relabeling of the planes $\overline{\mathcal{H}}\left(\mathbf{u}_{\mathbb{J}}, \tau_{M}^{\mathbb{J}}\right)$ and $\overline{\mathcal{H}}\left(\mathbf{u}_{\mathfrak{J}}, \nu_{M}^{\mathbb{J}}\right)$ associated with the plane slice. Now, let $\mathcal{F}\left(\mathbf{d}_{M-1}^{\mathbb{J}<}, \mathbf{P}[\mathbb{J}]\right)=\mathcal{G}_{M-1} \cap \overline{\mathcal{H}}\left(\mathbf{u}_{\mathbb{J}}, \phi_{M-1}^{\mathbb{J}}\right)$ and $\mathcal{F}\left(\mathbf{d}_{M-1}^{\mathbb{J}>}, \mathbf{P}[\mathbb{J}]\right)=\mathcal{G}_{M-1} \cap \overline{\mathcal{H}}\left(\mathbf{u}_{\mathbb{J}}, \psi_{M-1}^{\mathbb{J}}\right)$ denote the facets of $\mathcal{G}_{M-1}$ supported by the planes $\overline{\mathcal{H}}\left(\mathbf{u}_{\mathbb{J}}, \phi_{M-1}^{\mathbb{J}}\right)$ and $\overline{\mathcal{H}}\left(\mathbf{u}_{\mathbb{J}}, \psi_{M-1}^{\mathbb{J}}\right)$, respectively. The relabeling also induces a corresponding partition of the surface of $\mathcal{G}_{M-1}$ into the two essentially disjoint subsets of facets $\mathcal{B}_{\mathcal{G}_{M-1}}=\mathcal{B}_{\mathcal{G}_{M-1}}^{<} \cup \mathcal{B}_{\mathcal{G}_{M-1}}^{>}$ where

$$
\begin{aligned}
& \mathcal{B}_{\mathcal{G}_{M-1}}^{<}=\bigcup_{\mathbb{J} \in \mathfrak{C}^{2}(\langle M-1\rangle)} \mathcal{F}\left(\mathbf{d}_{M-1}^{\mathbb{J}<}, \mathbf{P}[\mathbb{J}]\right), \\
& \mathcal{B}_{\mathcal{G}_{M-1}}^{>}=\bigcup_{\mathbb{J} \in \mathfrak{C}^{2}(\langle M-1\rangle)} \mathcal{F}\left(\mathbf{d}_{M-1}^{\mathbb{J}>}, \mathbf{P}[\mathbb{J}]\right) .
\end{aligned}
$$

Next we consider the edges of $\mathcal{G}_{M-1}$. Let $\mathcal{E}=\mathcal{E}\left(\mathbf{o}, \mathbf{p}_{i}\right)$ be an edge of $\mathcal{G}_{M-1}$ where o denotes the origin of the edge and $\mathbf{p}_{i}$ the primary spanning the edge. Let $\mathcal{F}_{j}=\mathcal{F}\left(\mathbf{o}_{j},\left[\mathbf{p}_{i}, \mathbf{p}_{j}\right]\right)$ and $\mathcal{F}_{l}=\mathcal{F}\left(\mathbf{o}_{l},\left[\mathbf{p}_{i}, \mathbf{p}_{l}\right]\right)$ denote the two facets of $\mathcal{G}_{M-1}$ such that $\mathcal{E}=\mathcal{F}_{j} \cap \mathcal{F}_{l}$. Now for $q=j, l, \mathbf{o}_{q}-\mathbf{o}=\left(\left(\delta_{q}-1\right) / 2\right) \mathbf{p}_{q}$ where $\delta_{q} \in\{ \pm 1\}$ and $\delta_{q} \mathbf{p}_{q}$ is the feasible direction from the edge $\mathcal{E}$ along the facet $\mathcal{F}_{q}$. Now, assume without loss of generality (wlog) that $\operatorname{sgn}\left(\operatorname{det}\left(\left[\mathbf{p}_{i}, \delta_{j} \mathbf{p}_{j}, \delta_{l} \mathbf{p}_{l}\right]\right)\right)=1$, so that $\mathbf{p}_{i}, \delta_{j} \mathbf{p}_{j}, \delta_{l} \mathbf{p}_{l}$ form a positively oriented basis (otherwise, swap $j$ and $l$ ). Then from the convexity of $\mathcal{G}_{M-1}$, it follows that $\left(\mathbf{p}_{i} \otimes \delta_{j} \mathbf{p}_{j}\right)$ and $\left(\delta_{l} \mathbf{p}_{l} \otimes \mathbf{p}_{i}\right)$ define the inward pointing normals for the facets $\mathcal{F}_{j}$ and $\mathcal{F}_{l}$, respectively. Now denote $\delta_{j M}(\mathcal{E})=$ 
$\operatorname{sgn}\left(\operatorname{det}\left(\left[\mathbf{p}_{i}, \mathbf{p}_{M}, \delta_{j} \mathbf{p}_{j}\right]\right)\right)=\operatorname{sgn}\left(\left(\mathbf{p}_{i} \otimes \mathbf{p}_{M}\right)^{T} \delta_{j} \mathbf{p}_{j}\right)=$ $\operatorname{sgn}\left(\mathbf{u}_{[i, M]}^{T} \delta_{j} \mathbf{p}_{j}\right)=-\operatorname{sgn}\left(\left(\mathbf{p}_{i} \otimes \delta_{j} \mathbf{p}_{j}\right)^{T} \mathbf{p}_{M}\right)$ and $\delta_{l M}(\mathcal{E})=$ $\operatorname{sgn}\left(\operatorname{det}\left(\left[\mathbf{p}_{i}, \mathbf{p}_{M}, \delta_{l} \mathbf{p}_{l}\right]\right)\right)=\operatorname{sgn}\left(\left(\mathbf{p}_{i} \otimes \mathbf{p}_{M}\right)^{T} \delta_{l} \mathbf{p}_{l}\right)=$ $\operatorname{sgn}\left(\mathbf{u}_{[i, M]}^{T} \delta_{l} \mathbf{p}_{l}\right)=\operatorname{sgn}\left(\left(\delta_{l} \mathbf{p}_{l} \otimes \mathbf{p}_{i}\right)^{T} \mathbf{p}_{M}\right)$. Then we can see that we have four cases based on the values of $\delta_{j M}(\mathcal{E}), \delta_{l M}(\mathcal{E})$ :

1) If $\delta_{j M}(\mathcal{E})=1$ and $\delta_{l M}(\mathcal{E})=1, \mathcal{E}$ is the (local, and via convexity global) minimizer of $f_{i}(\mathbf{t})$ over $\mathbf{t} \in \mathcal{G}_{M-1}$ and $\mathcal{F}_{j} \in \mathcal{B}_{\mathcal{G}_{M-1}}^{>}, \mathcal{F}_{l} \in \mathcal{B}_{\mathcal{G}_{M-1}}^{<}$. Furthermore,

$$
\begin{aligned}
\left\{\mathbf{t} \in \mathcal{G}_{M-1} \mid f_{i}(\mathbf{t})=\tau^{[i, M]}\right\} & =\mathcal{G}_{M-1} \cap \overline{\mathcal{H}}\left(\mathbf{u}_{[i, M]}, \tau^{[i, M]}\right) \\
= & \mathcal{E}\left(\mathbf{d}_{M-1}^{[i . M]-}, \mathbf{p}_{i}\right)=\mathcal{E},
\end{aligned}
$$

so that $\overline{\mathcal{H}}\left(\mathbf{u}_{[i, M]}, \tau^{[i, M]}\right)$ is a supporting plane for $\mathcal{E}$ and $\delta_{q}=\left(1-2 \chi_{q}^{[i, M]-}\right)$ for $q=j, l$.

2) If $\delta_{j M}(\mathcal{E})=-1$ and $\delta_{l M}(\mathcal{E})=-1, \mathcal{E}$ is the (local, and via convexity global) maximizer of $f_{i}(\mathbf{t})$ over $\mathbf{t} \in \mathcal{G}_{M-1}$ and $\mathcal{F}_{j} \in \mathcal{B}_{\mathcal{G}_{M-1}}^{<}, \mathcal{F}_{l} \in \mathcal{B}_{\mathcal{G}_{M-1}}^{>}$. Furthermore,

$$
\begin{aligned}
&\left\{\mathbf{t} \in \mathcal{G}_{M-1} \mid f_{i}(\mathbf{t})=\nu^{[i, M]}\right\}=\mathcal{G}_{M-1} \cap \overline{\mathcal{H}}\left(\mathbf{u}_{[i, M]}, \nu^{[i, M]}\right) \\
&=\mathcal{E}\left(\mathbf{d}_{M-1}^{[i . M]+}, \mathbf{p}_{i}\right)=\mathcal{E},
\end{aligned}
$$

so that $\overline{\mathcal{H}}\left(\mathbf{u}_{[i, M]}, \nu^{[i, M]}\right)$ is a supporting plane for the $\mathcal{E}$ and $\delta_{q}=\left(1-2 \chi_{q}^{[i, M]+}\right)$ for $q=j, l$.

3) If $\delta_{j M}(\mathcal{E})=1$ and $\delta_{l M}(\mathcal{E})=-1, \mathcal{F}_{j}, \mathcal{F}_{l} \in \mathcal{B}_{\mathcal{G}_{M-1}}^{>}$and $\mathcal{E}$ is neither a maximizer nor a minimizer of $f_{i}(\mathbf{t})$ over $\mathbf{t} \in \mathcal{G}_{M-1}$.

4) If $\delta_{j M}(\mathcal{E})=-1$ and $\delta_{l M}(\mathcal{E})=1, \mathcal{F}_{j}, \mathcal{F}_{l} \in \mathcal{B}_{\mathcal{G}_{M-1}}^{<}$and $\mathcal{E}$ is neither a maximizer nor a minimizer of $f_{i}(\mathbf{t})$ over $\mathbf{t} \in \mathcal{G}_{M-1}$

Figure 8 illustrates these four cases geometrically. It follows that we can partition the edges of $\mathcal{G}_{M-1}$ into four sets

$$
\begin{aligned}
& \mathcal{E}_{\mathcal{G}_{M-1}^{<}}^{><}=\left\{\mathcal{E} \in \mathcal{W}_{\mathcal{G}_{M-1}} \mid \delta_{j M}(\mathcal{E})=\delta_{l M}(\mathcal{E})=1\right\}, \\
& \mathcal{E}_{\mathcal{G}_{M-1}>>}^{<>}=\left\{\mathcal{E} \in \mathcal{W}_{\mathcal{G}_{M-1}} \mid \delta_{j M}(\mathcal{E})=\delta_{l M}(\mathcal{E})=-1\right\}, \\
& \mathcal{E}_{\mathcal{G}_{M-1}}^{>>}=\left\{\mathcal{E} \in \mathcal{W}_{\mathcal{G}_{M-1}} \mid \delta_{j M}(\mathcal{E})=1=-\delta_{l M}(\mathcal{E})\right\}, \\
& \mathcal{E}_{\mathcal{G}_{M-1}<}^{<<}=\left\{\mathcal{E} \in \mathcal{W}_{\mathcal{G}_{M-1}} \mid \delta_{j M}(\mathcal{E})=-1=-\delta_{l M}(\mathcal{E})\right\} .
\end{aligned}
$$

Denote

$$
\begin{aligned}
\mathcal{A}_{M} & =\bigcup_{\mathbb{J} \in \mathfrak{C}^{2}(\langle M-1\rangle)} \mathcal{P}\left(\mathbf{c}_{M}^{\mathbb{J}}, \mathbf{P}[\mathbb{J}, M]\right), \\
\mathcal{A} & =\mathcal{G}_{M-1} \bigcup \mathcal{A}_{M} \\
& =\bigcup_{i=3}^{M}\left(\bigcup_{\mathbb{J} \in \mathfrak{C}^{2}(\langle i-1\rangle)} \mathcal{P}\left(\mathbf{c}_{i}^{\mathbb{J}}, \mathbf{P}[\mathbb{J}, i]\right)\right) .
\end{aligned}
$$

As a part of the induction step for establishing the first clause of the theorem, we show next that $\mathcal{A}=\mathcal{G}_{M}$ and that the sets in (73) are essentially disjoint. Suppose, $\mathbf{t} \in \mathcal{A}$. Then from (72), either $\mathbf{t} \in \mathcal{G}_{M-1} \subseteq \mathcal{G}_{M}$ or $\mathbf{t} \in \mathcal{A}_{M}$, i.e., there exists a $\mathbb{J}_{0} \in \mathfrak{C}^{2}(\langle M-1\rangle)$ such that $\mathbf{t} \in \mathcal{P}\left(\mathbf{c}_{M}^{\mathbb{J}_{0}}, \mathbf{P}\left[\mathbb{J}_{0}, M\right]\right)$. In the latter case, by Lemma $1, \boldsymbol{\beta}^{\mathbb{J}_{0}, M}=\mathbf{P}^{-1}\left[\mathbb{J}_{0}, M\right]\left(\mathbf{t}-\mathbf{c}_{M}^{\mathbb{J}_{0}}\right)$ is

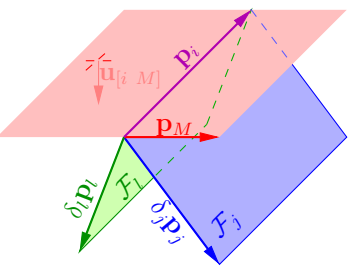

(a) $\mathcal{E} \in \mathcal{E}_{\mathcal{G}_{M-1}}^{><}$

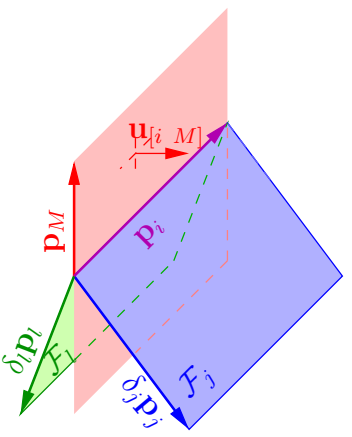

(c) $\mathcal{E} \in \mathcal{E}_{\mathcal{G}_{M-1}}^{>>}$

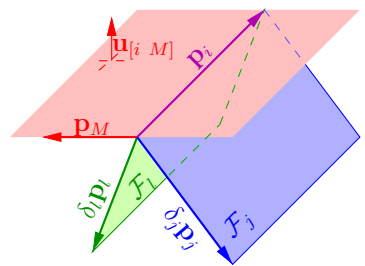

(b) $\mathcal{E} \in \mathcal{E}_{\mathcal{G}_{M-1}}^{<>}$

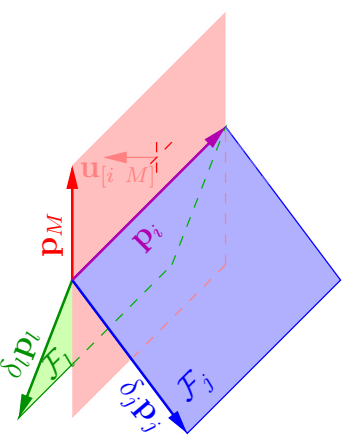

(d) $\mathcal{E} \in \mathcal{E}_{\mathcal{G}_{M-1}^{<}}^{<<}$
Fig. 8. Classification of edges of $\mathcal{G}_{M-1}$. An edge $\mathcal{E}$ of $\mathcal{G}_{M-1}$ spanned by the primary $\mathbf{p}_{i}$ is the intersection of two different facets $\mathcal{F}_{j}=\mathcal{F}\left(\mathbf{o}_{j},\left[\mathbf{p}_{i}, \mathbf{p}_{j}\right]\right)$ (plotted in blue) and $\mathcal{F}_{l}=\mathcal{F}\left(\mathbf{o}_{l},\left[\mathbf{p}_{i}, \mathbf{p}_{l}\right]\right)$ (plotted in green), with the common primary $\mathbf{p}_{i}$ (plotted in purple). The figure shows the facets using a positive orientation for the basis $\mathbf{p}_{i}, \delta_{j} \mathbf{p}_{j}, \delta_{l} \mathbf{p}_{l}$, where $\delta_{q} \in\{ \pm 1\}$ is defined by the fact that $\delta_{q} \mathbf{p}_{q}$ is the feasible direction from the edge $\mathcal{E}$ along the facet $\mathcal{F}_{q}$. The values $\delta_{j M}(\mathcal{E})=\operatorname{sgn}\left(\operatorname{det}\left(\left[\mathbf{p}_{i}, \mathbf{p}_{M}, \delta_{j} \mathbf{p}_{j}\right]\right)\right) \in\{-1,1\}$ and $\delta_{l M}(\mathcal{E})=\operatorname{sgn}\left(\operatorname{det}\left(\left[\mathbf{p}_{i}, \mathbf{p}_{M}, \delta_{l} \mathbf{p}_{l}\right]\right)\right) \in\{-1,1\}$ classify the edges into the four cases shown: (a) $\delta_{j M}(\mathcal{E})=\delta_{l M}(\mathcal{E})=1, \mathcal{E} \in \mathcal{E}_{\mathcal{G}_{M-1}}^{>}$, (b) $\delta_{j M}(\mathcal{E})=\delta_{l M}(\mathcal{E})=-1, \mathcal{E} \in \mathcal{E}_{\mathcal{G}_{M-1}>>},\left(\right.$ c) $\delta_{j M}(\mathcal{E})=1=-\delta_{l M}(\mathcal{E})$, $\mathcal{E} \in \mathcal{E}_{\mathcal{G}_{M-1}}^{>>}$, and (d) $\delta_{j M}(\mathcal{E})=-1=-\delta_{l M}(\mathcal{E}), \mathcal{E} \in \mathcal{E}_{\mathcal{G}_{M-1}}^{<<}$

the unique vector in $[0,1]^{3}$ such that $\mathbf{t}=\mathbf{P}\left[\mathbb{J}_{0}, M\right] \boldsymbol{\beta}^{\mathbb{J}_{0}, M}+\mathbf{c}_{M}^{\mathbb{J}_{0}}$. Now, by definition, $\mathbf{c}_{M}^{\mathbb{J}_{0}}=\mathbf{t}_{\mathbf{0}}+\mathbf{P} \boldsymbol{\alpha}_{M}^{\mathbb{J}_{0}}$, with

$$
\boldsymbol{\alpha}_{M}^{\mathbb{J}_{0}}=\chi^{+}\left(\mathbf{u}_{\mathbb{J}_{0}}^{T} \mathbf{p}_{M}\right) \chi_{M-1}^{\mathbb{J}_{0}+}+\chi^{-}\left(\mathbf{u}_{\mathbb{J}_{0}}^{T} \mathbf{p}_{M}\right) \chi_{M-1}^{\mathbb{J}_{0}-} .
$$

Observe that the definition of $\boldsymbol{\alpha}_{M}^{\mathbb{J}_{0}}$ ensures that the entries in this vector located at indices in $\left[\mathbb{J}_{0}, M\right]$ are zero. Using this observation and noting that $\mathbf{P}\left[\mathbb{J}_{0}, M\right]=\mathbf{P} \mathbf{I}_{K}\left[\mathbb{J}_{0}, M\right]$, we can see that $\boldsymbol{\alpha}=\boldsymbol{\alpha}_{M}^{\mathbb{J}_{0}}+\mathbf{I}_{K}\left[\mathbb{J}_{0}, M\right] \boldsymbol{\beta}_{M}^{\mathbb{J}_{0}}=\boldsymbol{\alpha}_{M}^{\mathbb{J}_{0}}+$ $\mathbf{I}_{K}\left[\mathbb{J}_{0}, M\right] \mathbf{P}^{-1}\left[\mathbb{J}_{0}, M\right]\left(\mathbf{t}-\mathbf{c}_{M}^{\mathbb{J}_{0}}\right)$ defines a feasible control vector in $[0,1]^{M}$ such that $\mathbf{t}=\mathbf{P} \boldsymbol{\alpha}+\mathbf{t}_{\mathbf{0}}=\mathbf{P}[\langle M\rangle] \boldsymbol{\alpha}+\mathbf{t}_{\mathbf{0}}$. It follows then that $\mathbf{t} \in \mathcal{G}_{M}$ and therefore $\mathcal{A} \subseteq \mathcal{G}_{M}$. Next suppose $\mathbf{t} \in \mathcal{G}_{M}$. Then from (58) it follows that $\mathbf{t}=\mathbf{P} \boldsymbol{\alpha}+\mathbf{t}_{\mathbf{0}}$ for some $\boldsymbol{\alpha} \in[0,1]^{M}$. If $\mathbf{t} \in \mathcal{G}_{M-1}$ then $\mathbf{t} \in \mathcal{A}$ because $\mathcal{G}_{M-1} \subset \mathcal{A}$. If on the other hand, $\mathbf{t} \notin \mathcal{G}_{M-1}$ then $\alpha_{M}>0$ and $\left(\mathbf{t}-\alpha_{M} \mathbf{p}_{M}\right) \in \mathcal{G}_{M-1}$. Let $\gamma$ denote the smallest $^{13}$ nonnegative real number such that $\mathbf{t}^{\prime}=\mathbf{t}-\gamma \mathbf{p}_{M}$ is in $\mathcal{G}_{M-1}$. Note that $0<\gamma \leq \alpha_{M} \leq 1$ and $\mathbf{t}^{\prime}$ lies on a surface facet of $\mathcal{G}_{M-1}$ such that $\mathbf{p}_{M}$ points outward from the facet. Specifically, there exists $\mathbb{J}_{\mathbf{t}} \in \mathfrak{C}^{2}(\langle M-1\rangle)$ such that either $\mathbf{t}^{\prime} \in \mathcal{F}\left(\mathbf{d}_{M-1}^{\mathbb{J}_{\mathbf{t}}-}, \mathbf{P}\left[\mathbb{J}_{\mathbf{t}}\right]\right)$ and $\mathbf{u}_{\mathbb{J}_{\mathbf{t}}}^{T} \mathbf{p}_{M}<0$, or $\mathbf{t}^{\prime} \in \mathcal{F}\left(\mathbf{d}_{M-1}^{\mathbb{t}_{\mathbf{t}}+}, \mathbf{P}\left[\mathbb{J}_{\mathbf{t}}\right]\right)$ and $\mathbf{u}_{\mathbb{J}_{\mathbf{t}}}^{T} \mathbf{p}_{M}>0$. Consider the case when $\mathbf{u}_{\mathbb{J}_{\mathrm{t}}}^{T} \mathbf{p}_{M}<0$ (the other case is similarly handled). Then $\mathbf{c}_{M}^{\mathbb{J}_{\mathbf{t}}}=\mathbf{d}_{M-1}^{\mathbb{J}_{\mathbf{t}}-}$ and $\mathbf{t} \in \mathcal{P}\left(\mathbf{c}_{M}^{\mathbb{J}_{\mathbf{t}}}, \mathbf{P}\left[\mathbb{J}_{\mathbf{t}}, M\right]\right) \subset \mathcal{A}_{M} \subset \mathcal{A}$. It

\footnotetext{
${ }^{13}$ The fact that $\mathcal{G}_{M-1}$ is closed and bounded ensures that $\gamma$ is well defined.
} 
follows that $\mathcal{G}_{M} \subset \mathcal{A}$, which in combination with the result shown earlier in this paragraph implies that $\mathcal{G}_{M}=\mathcal{A}$.

Next, using the first part of Lemma 1 we obtain an alternative representation for $\mathcal{P}\left(\mathbf{c}_{M}^{\mathbb{J}}, \mathbf{P}[\mathbb{J}, M]\right)$ in (71). Specifically, noting that for any $\mathbb{J}$ occurring in (71), we have $\mathbf{c}_{M}^{\mathbb{J}}=$ $\chi^{-}\left(\mathbf{u}_{\mathbb{J}}^{T} \mathbf{p}_{M}\right) \mathbf{d}_{M-1}^{\mathbb{J}^{-}}+\chi^{+}\left(\mathbf{u}_{\mathbb{J}}^{T} \mathbf{p}_{M}\right) \mathbf{d}_{M-1}^{\mathbb{J}^{+}}$, we can see that

$$
\begin{aligned}
\mathcal{P}\left(\mathbf{c}_{M}^{\mathbb{J}}, \mathbf{P}[\mathbb{J}, M]\right) & =\mathcal{H}\left(\mathbf{u}_{\mathbb{J}}, \kappa_{M}^{\mathbb{J}}, \eta_{M}^{\mathbb{J}}\right) \bigcap \\
& \left(\bigcap_{j \in \mathbb{J}} \mathcal{H}\left(\mathbf{u}_{[j, M]}, \bar{\kappa}_{i}^{[j, M]}, \bar{\eta}_{i}^{[j, M]}\right)\right),
\end{aligned}
$$

where $i=\mathbb{J} \backslash j, \bar{\kappa}_{i}^{[j, M]}=f_{j}\left(\mathbf{c}_{M}^{\mathbb{J}}+\chi^{-}\left(\mathbf{u}_{[j, M]}^{T} \mathbf{p}_{i}\right) \mathbf{p}_{i}\right)$, $\bar{\eta}_{i}^{[j, M]}=f_{j}\left(\mathbf{c}_{M}^{\mathbb{J}}+\chi^{+}\left(\mathbf{u}_{[j, M]}^{T} \mathbf{p}_{i}\right) \mathbf{p}_{i}\right)$. It follows that $\left[\bar{\kappa}_{i}^{[j, M]}, \bar{\eta}_{i}^{[j, M]}\right]$ is a non-empty interval with $f_{j}\left(\mathbf{c}_{M}^{\mathbb{J}}\right)$ and $f_{j}\left(\mathbf{c}_{M}^{\mathbb{J}}+\mathbf{p}_{i}\right)$ as its end points. Using (75) with the fact that the intervals in the right hand side (RHS) of (52) are essentially-disjoint, it follows that the parallelepipeds in the union on the RHS of (71) are essentially-disjoint with $\mathcal{G}_{M-1}$. From our prior characterization of $\tau_{M}^{[j, M]}$ and $\nu_{M}^{[j, M]}$ as the minima and maxima, respectively, of $f_{j}(\mathbf{t})$ over $\mathbf{t} \in \mathcal{G}_{M}$, it follows that for each $j \in\langle M-1\rangle,\left[\bar{\kappa}_{i}^{[j, M]}, \bar{\eta}_{i}^{[j, M]}\right] \subset$ $\left[\tau_{M}^{[j, M]}, \nu_{M}^{[j, M]}\right]$. Using the linearity of the functions $f_{j}$ along with (18)-(19) and (48)-(51), we also see that $\sum_{i \in\langle M-1\rangle \backslash j}\left(\bar{\eta}_{i}^{[j, M]}-\bar{\kappa}_{i}^{[j, M]}\right)=\mathbf{P}\left(\chi_{M}^{[j, M]+}-\chi_{M}^{[j, M]-}\right)=$ $\left(\nu_{M}^{[j, M]}-\tau_{M}^{[j, M]}\right)$, whereby it follows that the nonempty intervals $\left[\bar{\kappa}_{i}^{[j, M]}, \bar{\eta}_{i}^{[j, M]}\right], i \in\langle M-1\rangle \backslash j$ form an essentially disjoint partition of the interval $\left[\tau_{M}^{[j, M]}, \nu_{M}^{[j, M]}\right]$. It follows that each of the parallelepipeds in (71) and (73) are essentially disjoint and for each $j \in\langle M-1\rangle$, we have a sequence $\mathbb{I}_{j} \in \mathfrak{P}(\langle M-1\rangle \backslash j)$ such that

$$
\begin{aligned}
\tau^{[j, M]}= & \bar{\kappa}_{\mathbb{I}_{j}[1]}^{[j, M]}<\bar{\eta}_{\mathbb{I}_{j}[1]}^{[j, M]}=\bar{\kappa}_{\mathbb{I}_{j}[2]}^{[j, M]}<\bar{\eta}_{\mathbb{I}_{j}[2]}^{[j, M]} \cdots \\
& \bar{\kappa}_{\mathbb{I}_{j}[M-2]}^{[j, M]}<\bar{\eta}_{\mathbb{I}_{j}[M-2]}^{[j, M]}=\nu_{M}^{[j, M]}
\end{aligned}
$$

This completes the induction step for Clause 1 for Theorem 1 . Next let

$$
\mathcal{C}=\bigcap_{\mathbb{J} \in \mathfrak{C}^{2}(\langle M\rangle)} \mathcal{H}\left(\mathbf{u}_{\mathbb{J}}, \tau_{M}^{\mathbb{J}}, \nu_{M}^{\mathbb{J}}\right)
$$

As part of the induction to establish the second part of the theorem, we show next that $\mathcal{C}=\mathcal{G}_{M}$. First observe that if $\mathbf{y} \in \mathcal{G}_{M}$, then $\mathbf{y}=\mathbf{t}_{\mathbf{0}}+\mathbf{P}[\langle M\rangle] \boldsymbol{\alpha}$ for some $\boldsymbol{\alpha} \in[0,1]^{M}$. Using (18),(19),(50), and (51), we can deduce that for any $\mathbb{J} \in$ $\mathfrak{C}^{2}(\langle M\rangle), \mathbf{u}_{\sqrt{J}}^{T} \mathbf{y}-\tau_{M}^{\mathbb{J}}=\sum_{k=1}^{M}\left|\left(\alpha_{k}-\chi^{-}\left(\mathbf{u}_{\sqrt{J}}^{T} \mathbf{p}_{k}\right)\right) \mathbf{u}_{\sqrt{J}}^{T} \mathbf{p}_{k}\right|$ $\nu_{M}^{\mathbb{J}}=\sum_{k=1}^{M}\left|\left(\alpha_{k}-\chi^{+}\left(\mathbf{u}_{\mathbb{J}}^{T} \mathbf{p}_{k}\right)\right) \mathbf{u}_{\mathbb{J}}^{T} \mathbf{p}_{k}\right|$ and thus $\mathbf{u}_{\mathbb{J}}^{T} \mathbf{y} \in$ $\left[\tau_{M}^{\mathbb{J}}, \nu_{M}^{\mathbb{J}}\right]$. It follows that $\mathcal{G}_{M} \subset \mathcal{C}$. Next we show that $\mathcal{C} \subset \mathcal{G}_{M}$.
First observe that $\mathcal{G}_{M-1} \subset \mathcal{G}_{M} \subset \mathcal{C}$, therefore, we can write

$$
\begin{aligned}
\mathcal{G}_{M-1} & =\left(\bigcap_{i \in\langle M-1\rangle} \mathcal{H}\left(\mathbf{u}_{[i, M]}, \tau_{M}^{[i, M]}, \nu_{M}^{[i, M]}\right)\right) \bigcap \\
& \bigcap_{\mathbb{J} \in \mathfrak{C}^{2}(\langle M-1\rangle)} \mathcal{H}\left(\mathbf{u}_{\mathbb{J}}, \tau_{M-1}^{\mathbb{J}}, \nu_{M-1}^{\mathbb{J}}\right) .
\end{aligned}
$$

Now consider the polyhedron

$$
\begin{aligned}
\mathcal{C}_{M-1}^{*}= & \left(\bigcap_{i \in\langle M-1\rangle} \mathcal{H}\left(\mathbf{u}_{[i, M]}, \tau_{M}^{[i, M]}, \nu_{M}^{[i, M]}\right)\right) \bigcap \\
& \left(\bigcap_{\mathbb{J} \in \mathfrak{C}^{2}(\langle M-1\rangle)} \mathcal{H}^{>}\left(\mathbf{u}_{\mathbb{J}}, \psi_{M-1}^{\mathbb{J}}\right)\right) .
\end{aligned}
$$

From our characterization and partitioning of the facets and edges of $\mathcal{G}_{M-1}$, we see that for every $\mathbb{J} \in \mathfrak{C}^{2}(\langle M-1\rangle)$, the facet $\mathcal{F}_{\mathbb{J}}^{>}=\mathcal{G}_{M-1} \bigcap \overline{\mathcal{H}}^{>}\left(\mathbf{u}_{\mathbb{J}}, \psi_{M-1}^{\mathbb{J}}\right)$ lies in $\mathcal{B}_{\mathcal{G}_{M-1}}^{>}$and that the edges defining the boundary of the facet either

1) belong to $\mathcal{E}_{\mathcal{G}_{M-1}}^{>}$, in which case, the edge is the intersection of $\mathcal{F}_{\mathbb{J}}^{>}$with another facet $\mathcal{F}_{\mathbb{J}^{\prime}}^{>}=$ $\mathcal{G}_{M-1} \cap \overline{\mathcal{H}}^{>}\left(\mathbf{u}_{\mathbb{J}^{\prime}}, \psi_{M-1}^{\mathbb{J}^{\prime}}\right) \in \mathcal{B}_{\mathcal{G}_{M-1}}^{>}$, or

2) belongs to $\mathcal{E}_{\mathcal{G}_{M-1}}^{<>}$or $\mathcal{E}_{\mathcal{G}_{M-1}}^{<}$, in which case, the edge is the intersection of $\mathcal{F}_{\mathbb{J}}^{>}$with a supporting plane $\overline{\mathcal{H}}\left(\mathbf{u}_{[i, M]}, \tau_{M}^{[i, M]}\right)$ or $\overline{\mathcal{H}}\left(\mathbf{u}_{[i, M]}, \nu_{M}^{[i, M]}\right)$.

In either case, $\mathcal{C}_{M-1}^{*} \cap \overline{\mathcal{H}}^{>}\left(\mathbf{u}_{\mathbb{J}}, \psi_{M-1}^{\mathbb{J}}\right)=\mathcal{F}_{\mathbb{J}}^{>}$, whereby we can conclude that the boundary of the polyhedron $\mathcal{C}_{M-1}^{*}$ satisfies

$$
\begin{array}{r}
\mathcal{B}_{\mathcal{C}_{M-1}^{*}} \subset \bigcup_{\mathbb{J} \in \mathfrak{C}^{2}(\langle M-1\rangle)} \mathcal{F}\left(\mathbf{d}^{\mathbb{J}>}, \mathbf{P}[\mathbb{J}]\right) \bigcup \\
\bigcup_{i \in\langle M-1\rangle}\left(\overline{\mathcal{H}}\left(\mathbf{u}_{[i, M]}, \tau_{M}^{[i, M]}\right) \bigcup \overline{\mathcal{H}}\left(\mathbf{u}_{[i, M]}, \nu_{M}^{[i, M]}\right)\right) .
\end{array}
$$

Note that $\mathcal{G}_{M-1} \subset \mathcal{C}_{M-1}^{*}$ and therefore $\mathcal{C}_{M-1}^{*} \neq \phi$. Now denoting

$$
\begin{aligned}
\mathcal{Y}_{M}= & \left(\left(\bigcap_{\mathbb{J} \in \mathfrak{C}^{2}(\langle M-1\rangle)} \mathcal{H}\left(\mathbf{u}_{\mathbb{J}}, \kappa_{M}^{\mathbb{J}}, \eta_{M}^{\mathbb{J}}\right)\right) \bigcap\right. \\
& \left.\left(\bigcap_{i \in\langle M-1\rangle} \mathcal{H}\left(\mathbf{u}_{[i, M]}, \tau_{M}^{[i, M]}, \nu_{M}^{[i, M]}\right)\right)\right),
\end{aligned}
$$

we see that $\mathcal{C}=\mathcal{G}_{M-1} \cup \mathcal{Y}_{M}$. Now suppose $\mathbf{y} \in \mathcal{C}$. If $\mathbf{y} \in \mathcal{G}_{M-1}$ then $\mathbf{y} \in \mathcal{G}_{M}$ because $\mathcal{G}_{M-1} \subseteq \mathcal{G}_{M}$. On the other hand, if $\mathbf{y} \notin \mathcal{G}_{M-1}$ then $\mathbf{y} \in \mathcal{Y}_{M}$ and $\mathbf{y} \notin \mathcal{C}_{M-1}^{*}$. Now consider $\mathbf{z}=\mathbf{y}-\mathbf{p}_{M}$. Then, for every $i \in\langle M-1\rangle, \mathbf{u}_{[i, M]}^{T} \mathbf{z}=$ $\mathbf{u}_{[i, M]}^{T} \mathbf{y} \in\left[\tau_{M}^{[i, M]}, \nu_{M}^{[i, M]}\right]$ and for every $\mathbb{J} \in \mathfrak{C}^{2}(\langle M-1\rangle)$, $\mathbf{u}_{\mathbb{J}}^{T} \mathbf{z}=\mathbf{u}_{J}^{T} \mathbf{y}-\operatorname{sgn}\left(\mathbf{u}_{J}^{T} \mathbf{p}_{M}\right)\left(\eta_{M}^{\mathbb{J}}-\kappa_{M}^{\mathbb{J}}\right)$, from which it follows that $\mathbf{z} \in \mathcal{H}^{>}\left(\mathbf{u}_{\mathbb{J}}, \psi_{M-1}^{\mathbb{J}}\right)$. Thus $\mathbf{z} \in \mathcal{C}_{M-1}^{*}$. Since $\mathcal{C}_{M-1}^{*}$ is a convex set and $\mathbf{y} \notin \mathcal{C}_{M-1}^{*}$ and $\mathbf{y}-\mathbf{p}_{M} \in \mathcal{C}_{M-1}^{*}$, there exists a smallest $0<\gamma<1$ such that $\mathbf{z}^{*}=\mathbf{y}-\gamma \mathbf{p}_{M} \in \mathcal{B}_{\mathcal{C}_{M-1}^{*}}$. Noting that $\mathbf{u}_{[i, M]}^{T} \mathbf{p}_{M}=0$ for all $i \in\langle M-1\rangle$, from (80), we can conclude that $\mathbf{z}^{*} \in \bigcup_{\mathbb{J} \in \mathfrak{C}^{2}(\langle M-1\rangle)} \mathcal{F}\left(\mathbf{d}_{M-1}^{\mathbb{J}\rangle}, \mathbf{P}[\mathbb{J}]\right)$. It 
then follows that $\mathbf{y}=\mathbf{z}^{*}+\gamma \mathbf{p}_{M} \in \mathcal{P}\left(\mathbf{d}_{M-1}^{\mathbb{J}>}, \mathbf{P}[\mathbb{J}, M]\right)=$ $\mathcal{P}\left(\mathbf{c}_{M}^{\mathbb{J}}, \mathbf{P}[\mathbb{J}, M]\right) \subset \mathcal{G}_{M}$. Thus $\mathcal{C} \subset \mathcal{G}_{M}$ and combining with our prior result we have $\mathcal{C}=\mathcal{G}_{M}$. The facts that $\mathcal{C}$ is a polyhedron and that $\mathcal{G}_{M}$, being an affine map of the closed and bounded unit hypercube, is a closed and bounded set then imply that $\mathcal{G}_{M}$ is a polytope that can be expressed as the convex hull of its vertices. This completes the induction step for Clause 2 for Theorem 1.

Now using the fact that $\mathcal{C}=\mathcal{G}_{M}$ along with Lemma 2 and 3, it follows that the boundary of $\mathcal{G}_{M}$ is

$$
\mathcal{B}_{\mathcal{G}_{M}}=\bigcup_{\mathbb{J} \in \mathfrak{C}^{2}(\langle M\rangle)}\left(\mathcal{F}\left(\mathbf{d}_{M}^{\mathbb{J}^{-}}, \mathbf{P}[\mathbb{J}]\right) \cup \mathcal{F}\left(\mathbf{d}_{M}^{\mathbb{J}^{+}}, \mathbf{P}[\mathbb{J}]\right)\right),
$$

with

$$
\begin{aligned}
& \mathcal{F}\left(\mathbf{d}_{M}^{\mathbb{J}^{-}}, \mathbf{P}[\mathbb{J}]\right)=\mathcal{G}_{M} \bigcap \overline{\mathcal{H}}\left(\mathbf{u}_{\mathbb{I}}, \tau_{M}^{\mathbb{J}}\right), \\
& \mathcal{F}\left(\mathbf{d}_{M}^{\mathbb{J}^{+}}, \mathbf{P}[\mathbb{J}]\right)=\mathcal{G}_{M} \bigcap \overline{\mathcal{H}}\left(\mathbf{u}_{\mathbb{J}}, \nu_{M}^{\mathbb{J}}\right) .
\end{aligned}
$$

representing a pair of congruent parallelogram facets with supporting planes $\overline{\mathcal{H}}\left(\mathbf{u}_{\mathbb{J}}, \tau_{M}^{\mathbb{J}}\right)$ and $\overline{\mathcal{H}}\left(\mathbf{u}_{\mathbb{J}}, \nu_{M}^{\mathbb{J}}\right)$, respectively, for each $\mathbb{J} \in \mathfrak{C}^{2}(\langle M\rangle)$. Thus $\mathcal{G}_{M}$ has $\left(\begin{array}{c}M \\ 2\end{array}\right)$ pairs of parallelogram facets. Using the preceding characterization of the boundary of $\mathcal{G}_{M}$ with Lemma 2 and 3 we also immediately see that a tristimulus $\mathbf{t} \in \mathcal{G}_{M}$ has a unique control vector iff $\mathbf{t} \in \mathcal{B}_{\mathcal{G}_{M}}$, establishing Clause 6 for Theorem 1.

Next we revisit the essentially disjoint set of parallelepipeds in the set $\mathcal{A}_{M}$ in (71) that are added in $\mathcal{G}_{M}$ over $\mathcal{G}_{M-1}$. From the defining expressions, we can see that for each $\mathbb{J} \in \mathfrak{C}^{2}(\langle M-1\rangle)$, we have $\mathbf{c}_{M}^{\mathbb{J}}=\mathbf{d}_{M-1}^{\mathbb{J}\rangle}$, i.e., the origin of the parallelepiped $\mathcal{P}\left(\mathbf{c}_{M}^{\mathbb{J}}, \mathbf{P}[\mathbb{J}, M]\right)$ and the origin of the facet $\mathcal{F}\left(\mathbf{d}_{M-1}^{\mathbb{J}>}, \mathbf{P}[\mathbb{J}]\right)$ are identical. It follows that there is a one-toone correspondence that relates, for each $\mathbb{J} \in \mathfrak{C}^{2}(\langle M-1\rangle)$, the facet $\mathcal{F}\left(\mathbf{d}_{M-1}^{\mathbb{J}>}, \mathbf{P}[\mathbb{J}]\right)$ of $\mathcal{G}_{M-1}$ and the parallelepiped $\mathcal{P}\left(\mathbf{c}_{M}^{\mathbb{J}}, \mathbf{P}[\mathbb{J}, M]\right)$ added in $\mathcal{G}_{M}$, with $\mathcal{P}\left(\mathbf{c}_{M}^{\mathbb{J}}, \mathbf{P}[\mathbb{J}, M]\right)=$ $\left\{\hat{\mathbf{t}}+\alpha_{M} \mathbf{p}_{M} \mid \hat{\mathbf{t}} \in \mathcal{F}\left(\mathbf{d}_{M-1}^{\mathbb{J}>}, \mathbf{P}[\mathbb{J}]\right), 0 \leq \alpha_{M} \leq 1\right\}$. It also follows that for each $\mathbb{J} \in \mathfrak{C}^{2}(\langle M-1\rangle)$, the parallelepiped $\mathcal{P}\left(\mathbf{c}_{M}^{\mathbb{J}}, \mathbf{P}[\mathbb{J}, M]\right)$ extends the sequence of $(M-3)$ parallelepipeds in $\mathcal{G}_{M-1}$ that include the primaries $\mathbf{P}[\mathrm{J}]$ and where the intersection of successive parallelepipeds is a common facet of both parallelepipeds spanned by the primaries $\mathbf{P}[\mathrm{J}]$ to a sequence of $(M-2)$ parallelepipeds in $\mathcal{G}_{M-1}$, where the same properties hold (If $\mathbf{u}_{\mathbb{J}}^{T} \mathbf{p}_{M}>0\left(\mathbf{u}_{\mathbb{J}}^{T} \mathbf{p}_{M}<0\right)$ the sequence $\mathbb{I}$ in Clause 9 for Theorem 1 for $\mathcal{G}_{M-1}$ is replaced by the sequence $[\mathbb{I}, M]([M, \mathbb{I}])$ for $\mathcal{G}_{M}$.).

Now, for a given $j \in\langle M-1\rangle$, consider the $\langle M-2\rangle$ parallelepipeds $\mathcal{P}\left(\mathbf{c}_{M}^{\mathbb{J}}, \mathbf{P}[\mathbb{J}, M]\right), \mathbb{J} \in \mathfrak{C}^{2}(\langle M-1\rangle), j \in$ $\mathbb{J}$ and their corresponding facets $\mathcal{F}\left(\mathbf{d}_{M-1}^{\mathbb{J}>}, \mathbf{P}[\mathbb{J}]\right), \mathbb{J} \in$ $\mathfrak{C}^{2}(\langle M-1\rangle), j \in \mathbb{J}$. Let $\mathbb{J}_{1}$ denote the pair of indices $j$ and $\mathbb{I}_{j}[1]$ then, from (76), we see that $\tau_{M}^{[j, M]}=\bar{\kappa}_{\mathbb{I}_{j}[1]}^{[j, M]}$ and the parallelepiped $\mathcal{P}\left(\mathbf{c}_{M}^{\mathbb{J}_{1}}, \mathbf{P}\left[\mathbb{J}_{1}, M\right]\right)$ has the corresponding facet $\mathcal{F}\left(\mathbf{d}_{M-1}^{\mathbb{J}_{1}>}, \mathbf{P}\left[\mathbb{J}_{1}\right]\right)$, over one of whose $\mathbf{p}_{j}$ edges the function $f_{j}(\mathbf{t})$ achieves the minimum value of
$\tau_{M}^{[j, M]}$. The other $\mathbf{p}_{j}$ edge of the facet $\mathcal{F}\left(\mathbf{d}_{M-1}^{\mathbb{J}_{1}>}, \mathbf{P}\left[\mathbb{J}_{1}\right]\right)$ lies in the plane $\overline{\mathcal{H}}\left(\mathbf{u}_{[j, M]}, \bar{\kappa}_{\mathbb{I}_{j}[1]}^{[j, M]}\right)$ is non-extremal with respect to the function $f_{j}(\mathbf{t})$ and from (69) we see that the edge is therefore shared with another facet $\mathcal{F}_{2}$ of $\mathcal{G}_{M-1}$ from the set $\mathcal{F}\left(\mathbf{d}_{M-1}^{\mathbb{J}>}, \mathbf{P}[\mathbb{J}]\right), \mathbb{J} \in \mathfrak{C}^{2}(\langle M-1\rangle), j \in$ $\mathbb{J}, \mathbb{J} \neq \mathbb{J}_{1}$. The corresponding parallelepiped $\mathcal{P}_{2}$ (from the set $\left.\mathcal{P}\left(\mathbf{c}_{M}^{\mathbb{J}}, \mathbf{P}[\mathbb{J}, M]\right), \mathbb{J} \in \mathfrak{C}^{2}(\langle M-1\rangle), j \in \mathbb{J}, \mathbb{J} \neq \mathbb{J}_{1}\right)$ has a facet supported by the plane $\overline{\mathcal{H}}\left(\mathbf{u}_{[j, M]}, \bar{\kappa}_{\mathbb{I}_{j}[1]}^{[j, M]}\right)$. From (76), we note that $\bar{\eta}_{\mathbb{I}_{j}[2]}^{[j, M]}=\bar{\kappa}_{\mathbb{I}_{j}[1]}^{[j, M]}$ and the only parallelepiped from the set $\mathcal{P}\left(\mathbf{c}_{M}^{\mathbb{J}}, \mathbf{P}[\mathbb{J}, M]\right), \mathbb{J} \in \mathfrak{C}^{2}(\langle M-1\rangle), j \in \mathbb{J}, \mathbb{J} \neq \mathbb{J}_{1}$ that has a facet supported by the plane $\overline{\mathcal{H}}\left(\mathbf{u}_{[i, M]}, \bar{\kappa}_{\mathbb{I}_{j}[1]}^{[i, M]}\right)$ is the parallelepiped $\mathcal{P}\left(\mathbf{c}_{M}^{\mathbb{J}_{2}}, \mathbf{P}\left[\mathbb{J}_{2}, M\right]\right)$, where $j$ and $\mathbb{I}_{j}[2]$ are the elements of $\mathbb{J}_{2}$. Hence $\mathcal{P}_{2}=\mathcal{P}\left(\mathbf{c}_{M}^{\mathbb{J}_{2}}, \mathbf{P}\left[\mathbb{J}_{2}, M\right]\right)$ and $\mathcal{F}_{2}=\mathcal{F}\left(\mathbf{d}_{M-1}^{\mathbb{J}_{2}>}, \mathbf{P}\left[\mathbb{J}_{2}\right]\right)$. The process can be continued for $l=2,3, \ldots(M-3)$, where denoting $\mathbb{J}_{l+1}$ as the pair of indices $j$ and $\mathbb{I}_{j}[l+1]$, we conclude that the facet $\mathcal{F}\left(\mathbf{d}_{M-1}^{\mathbb{J}_{l}>}, \mathbf{P}\left[\mathbb{J}_{l}\right]\right)$ shares a $\mathbf{p}_{j}$ edge with the facet $\mathcal{F}\left(\mathbf{d}_{M-1}^{\mathbb{J}_{l+1}>}, \mathbf{P}\left[\mathbb{J}_{l+1}\right]\right)$ contained in the facet supporting plane $\overline{\mathcal{H}}\left(\mathbf{u}_{[i, M]}, \bar{\kappa}_{\mathbb{I}_{j}[l+1]}^{[i, M]}\right)$ of the associated parallelepiped $\mathcal{P}\left(\mathbf{c}^{\mathbb{J}_{l+1}}, \mathbf{P}\left[\mathbb{J}_{l+1}, M\right]\right)$. Relating the origins of the chain of facets with the shared edges, we obtain $\mathbf{c}_{M}^{\mathbb{J}_{l}}=\mathbf{d}_{M-1}^{\mathbb{J}_{l}>}=\mathbf{d}_{M-1}^{\mathbb{J}_{l-1}>}+\operatorname{sgn}\left(\mathbf{u}_{[j, M]}^{T} \mathbf{p}_{\mathbb{I}_{j}[l]}\right) \mathbf{p}_{\mathbb{I}_{j}[l]}=$ $\mathbf{c}_{M}^{\mathbb{J}_{l-1}}+\operatorname{sgn}\left(\mathbf{u}_{[j, M]}^{T} \mathbf{p}_{\mathbb{I}_{j}[l]}\right) \mathbf{p}_{\mathbb{I}_{j}[l]}, l=2,3, \ldots M-2$. We can then see that for each $j \in\langle M-1\rangle$, the subset of $(M-2)$ parallelepipeds in the set $\mathcal{A}_{M}$ in (71) that include $\mathbf{p}_{j}$ and $\mathbf{p}_{M}$ among their spanning primaries form a chain in which the $l$ and $(l+1)^{\text {th }}$ parallelepipeds in the chain intersect in a common facet spanned by primaries $\mathbf{p}_{j}$ and $\mathbf{p}_{M}$, specifically the $M$ primary equivalent of (34) holds, viz.,

$$
\begin{array}{r}
\mathcal{P}\left(\mathbf{c}_{M}^{\mathbb{J}_{l}}, \mathbf{P}\left[\mathbb{J}_{l} \mid M\right]\right) \bigcap \overline{\mathcal{H}}\left(\mathbf{u}_{[j, M]}, \bar{\eta}_{\mathbb{I}_{j}[l]}^{[j, M]}\right)= \\
\mathcal{P}\left(\mathbf{c}_{M}^{\mathbb{J}_{l+1}}, \mathbf{P}\left[\mathbb{J}_{l+1} \mid M\right]\right) \bigcap \overline{\mathcal{H}}\left(\mathbf{u}_{[j, M]}, \bar{\kappa}_{\mathbb{I}_{j}[l+1]}^{[j, M]}\right) .
\end{array}
$$

Except for the facets supported by the planes $\overline{\mathcal{H}}\left(\mathbf{u}_{[j, M]}, \tau_{M}^{[j, M]}\right)$ and $\overline{\mathcal{H}}\left(\mathbf{u}_{[j, M]}, \nu_{M}^{[j, M]}\right)$ all other facets of parallelepipeds $\mathcal{P}\left(\mathbf{c}_{M}^{\mathbb{J}}, \mathbf{P}[\mathbb{J}, M]\right), \mathbb{J} \in \mathfrak{C}^{2}(\langle M-1\rangle), j \in \mathbb{J}$ spanned by the primaries $\mathbf{p}_{j}$ and $\mathbf{p}_{M}$ occur in pairs of parallelepipeds that lie on either side of the facets ensuring that the relative interior of these facets is in the relative interior of $\mathcal{G}_{M}$. Combining with our prior conclusion for $\mathbb{J} \in \mathfrak{C}^{2}(\langle M-1\rangle)$ this completes the induction step for Clause 9 for Theorem 1 .

Consider next the relation between the boundaries of $\mathcal{G}_{M}$ and $\mathcal{G}_{M-1}$, which can be appreciated from the example illustrated in Fig. 9. By recalling that the planes $\overline{\mathcal{H}}\left(\mathbf{u}_{\mathbb{J}}, \phi_{M-1}^{\mathbb{J}}\right)$ and $\mathbf{p}_{M}+\overline{\mathcal{H}}\left(\mathbf{u}_{\mathbb{J}}, \psi_{M-1}^{\mathbb{J}}\right)$ provide a relabeling of the support- 
ing planes $\overline{\mathcal{H}}\left(\mathbf{u}_{\mathbb{J}}, \tau_{M}^{\mathbb{J}}\right)$ and $\overline{\mathcal{H}}\left(\mathbf{u}_{\mathbb{J}}, \nu_{M}^{\mathbb{J}}\right)$, we can write

$$
\begin{aligned}
\mathcal{B}_{\mathcal{G}_{M}} & =\left[\bigcup_{\mathbb{J} \in \mathfrak{C}^{2}(\langle M-1\rangle)}\left(\mathcal{F}\left(\mathbf{d}_{M}^{\mathbb{J}^{-}}, \mathbf{P}[\mathbb{J}]\right) \cup \mathcal{F}\left(\mathbf{d}_{M}^{\mathbb{J}^{+}}, \mathbf{P}[\mathbb{J}]\right)\right) \cup\right. \\
& {\left[\bigcup_{i \in\langle M-1\rangle}\left(\mathcal{F}\left(\mathbf{d}_{M}^{[i, M]-}, \mathbf{P}[i, M]\right) \cup \mathcal{F}\left(\mathbf{d}_{M}^{[i, M]+}, \mathbf{P}[i, M]\right)\right)\right] } \\
& {\left[\bigcup_{\mathbb{J} \in \mathfrak{C}^{2}(\langle M-1\rangle)}\left(\mathcal{F}\left(\mathbf{d}_{M}^{\mathbb{J}<}, \mathbf{P}[\mathbb{J}]\right) \cup\left(\mathbf{p}_{M}+\mathcal{F}\left(\mathbf{d}_{M}^{\mathbb{J}>}, \mathbf{P}[\mathbb{J}]\right)\right)\right)\right] } \\
& {\left[\bigcup_{j \in\langle M-1\rangle}\left(\mathcal{F}\left(\mathbf{d}_{M}^{[j, M]-}, \mathbf{P}[j, M]\right) \cup \mathcal{F}\left(\mathbf{d}_{M}^{[j, M]+}, \mathbf{P}[j, M]\right)\right)\right] }
\end{aligned}
$$

where the sets involved are essentially disjoint and therefore involve distinct facets. Additionally, we can see that for each $\mathbb{J} \in \mathfrak{C}^{2}(\langle M-1\rangle)$, edges of the facet $\mathcal{F}\left(\mathbf{d}_{M}^{\mathbb{J}<}, \mathbf{P}[\mathbb{J}]\right)$ (alternatively, $\left.\mathbf{p}_{M}+\mathcal{F}\left(\mathbf{d}_{M}^{\mathbb{J}>}, \mathbf{P}[\mathbb{J}]\right)\right)$ that are in $\mathcal{E}_{\mathcal{G}_{M-1}}^{<<}$(alternatively, $\mathcal{E}_{\mathcal{G}_{M-1}}^{>>}$) are supported by the (alternatively, $\mathbf{p}_{M}$ translated versions of the) same planes in $\mathcal{G}_{M}$ as they were in $\mathcal{G}_{M-1}$ and edges that are in $\mathcal{E}_{\mathcal{G}_{M-1}}^{><}$or $\mathcal{E}_{\mathcal{G}_{M-1}}^{<>}$are supported in $\mathcal{G}_{M}$ by either the plane $\overline{\mathcal{H}}\left(\mathbf{u}_{[j, M]}, \tau_{M}^{[j, M]}\right)$ or $\overline{\mathcal{H}}\left(\mathbf{u}_{[j, M]}, \nu_{M}^{[j, M]}\right)$ where $j$ denotes the index of the primary spanning the edge. Also, for each $j \in\langle M-1\rangle$, for each of the facets $\mathcal{F}\left(\mathbf{d}_{M}^{[j, M]-}, \mathbf{P}[j, M]\right)$ and $\mathcal{F}\left(\mathbf{d}_{M}^{[j, M]+}, \mathbf{P}[j, M]\right)$,

- edges spanned by the primary $\mathbf{p}_{j}$ are seen (using Lemma 1 and our previous organization of the parallelepipeds into chains) to be intersections of these facets with a facet $\mathcal{F}\left(\mathbf{d}_{M}^{\mathbb{J}^{-}}, \mathbf{P}[\mathbb{J}]\right)$ or $\mathcal{F}\left(\mathbf{d}_{M}^{\mathbb{J}^{+}}, \mathbf{P}[\mathbb{J}]\right)$ with $\mathbb{J} \in \mathfrak{C}^{2}(\langle M-1\rangle), j \in \mathbb{J}$, and the edges are therefore supported by the corresponding planes $\overline{\mathcal{H}}\left(\mathbf{u}_{\mathbb{J}}, \tau_{M}^{\mathbb{J}}\right)$ or $\overline{\mathcal{H}}\left(\mathbf{u}_{\mathbb{J}}, \nu_{M}^{\mathbb{J}}\right)$, and

- edges spanned by the primary $\mathbf{p}_{M}$ are the edges of a parallelepiped in $\mathcal{A}_{M}$ defined in (71) and supported (as edges of the facet polytope) by one of the planes $\overline{\mathcal{H}}\left(\mathbf{u}_{[i, M]}, \tau_{M}^{[i, M]}\right)$ and $\overline{\mathcal{H}}\left(\mathbf{u}_{[i, M]}, \nu_{M}^{[i, M]}\right)$ for some $i \in$ $\langle M-1\rangle, i \neq j$. Specifically, consider an edge $\mathcal{E}$ of the facet $\mathcal{F}\left(\mathbf{d}_{M}^{[j, M]-}, \mathbf{P}[j, M]\right)$ (the case for an edge of the facet $\mathcal{F}\left(\mathbf{d}_{M}^{[j, M]+}, \mathbf{P}[j, M]\right)$ follows an analogous argument) spanned by the primary $\mathbf{p}_{M}$. The facet $\mathcal{F}\left(\mathbf{d}_{M}^{[j, M]-}, \mathbf{P}[j, M]\right)$ of $\mathcal{G}_{M}$ is also a facet of the parallelepiped $\mathcal{P}\left(\mathbf{c}_{\mathbb{I}_{j}[1]}^{\mathbb{J}}, \mathbf{P}[\mathbb{J}, M]\right)$ where $j$ and $\mathbb{I}_{j}[1]$ are the two indices in $\mathbb{J}$. Now by Lemma 1 , in the parallelepiped $\mathcal{P}\left(\mathbf{c}_{\mathbb{I}_{j}[1]}^{\mathbb{J}}, \mathbf{P}[\mathbb{J}, M]\right)$ edge $\mathcal{E}$ is the intersection of the facet $\mathcal{F}\left(\mathbf{d}_{M}^{[j, M]-}, \mathbf{P}[j, M]\right)$ of $\mathcal{G}_{M}$ with another facet $\mathcal{F}_{2}$ that is spanned by $\mathbf{p}_{M}$ and $\mathbf{p}_{\mathbb{I}_{j}[1]}$. If $\mathcal{F}_{2}$ is also a facet of $\mathcal{G}_{M}$, then its supporting plane in the parallelepiped $\mathcal{P}\left(\mathbf{c}_{\mathbb{I}_{j}[1]}^{\mathbb{J}}, \mathbf{P}[\mathbb{J}, M]\right)$ also serves as the supporting plane for the edge $\mathcal{E}$ in the facet $\mathcal{F}\left(\mathbf{d}_{M}^{[j, M]-}, \mathbf{P}[j, M]\right)$ of $\mathcal{G}_{M}$. If on the other hand, the $\mathcal{F}_{2}$ is not a facet of $\mathcal{G}_{M}$,

then from the fact that the parallelepipeds in $\mathcal{G}_{M}$ that include the primaries $\mathbf{p}_{M}$ and $\mathbf{p}_{\mathbb{I}_{j}[1]}$ are arranged in a chain with only the facets located in planes extremal for $f_{\mathbb{I}_{j}[1]}(\mathbf{t})$ spanned by the primaries $\mathbf{p}_{M}$ and $\mathbf{p}_{\mathbb{I}_{j}[1]}$ exposed as surfaces of the gamut (recall (85)), it follows that there is a parallelepiped $\mathcal{P}_{2}$ that shares the facet $\mathcal{F}_{2}$ with the parallelepiped $\mathcal{P}\left(\mathbf{c}_{\mathbb{I}_{j}[1]}^{\mathbb{J}}, \mathbf{P}[\mathbb{J}, M]\right)$. By Lemma 1, in the parallelepiped $\mathcal{P}_{2}$ the edge $\mathcal{E}$ is the intersection of $\mathcal{F}_{2}$ with another facet $\mathcal{F}_{3}$, and because the edge is an edge of $\mathcal{G}_{M}$ and every edge is contained in a surface facet, it follows that $\mathcal{F}_{3}$ is a facet of $\mathcal{G}_{M}$ that intersects the facet $\mathcal{F}\left(\mathbf{d}_{M}^{[j, M]-}, \mathbf{P}[j, M]\right)$ in the edge $\mathcal{E}$ and the supporting plane for the facet $\mathcal{F}_{3}$ serves as a supporting plane for the edge $\mathcal{E}$ for the facet $\mathcal{F}\left(\mathbf{d}_{M}^{[j, M]-}, \mathbf{P}[j, M]\right)$.

Thus we have shown that every edge of a facet spanned by a primary $\mathbf{p}_{j}$ in $\mathcal{G}_{M}$ is supported by a plane $\overline{\mathcal{H}}\left(\mathbf{u}_{\bar{J}}, \tau^{\overline{\mathbb{J}}}\right)$ or $\overline{\mathcal{H}}\left(\mathbf{u}_{\overline{\mathbb{J}}}, \nu^{\overline{\mathbb{J}}}\right)$ for some $\overline{\mathbb{J}} \in \mathfrak{C}^{2}(\langle M\rangle)$ such that $j \in \overline{\mathbb{J}}$, completing the induction step for Clause 3 for Theorem 1. A direct implication of the clause is that every edge is contained in precisely two facets and is the intersection of the two facets that contain it. Therefore the number of distinct edges in $\mathcal{G}_{M}$ is $2 M(M-1)$. Furthermore, by combining the fact that the control values for tristimuli on the facets are unique and that those for the vertices of the facets take values only in $\{0,1\}$, we also see that any $\mathbf{t}$ is a vertex of $\mathcal{G}_{M}$ iff it is a vertex of one of the facets forming the boundary of $\mathcal{G}_{M}$. Using Euler's formula [16, pp. 123] relating the number of facets, edges, and vertices for a polytope in $\mathbb{R}^{3}$, we see that the number of distinct vertices in $\mathcal{G}_{M}$ is $M(M-1)+2$.

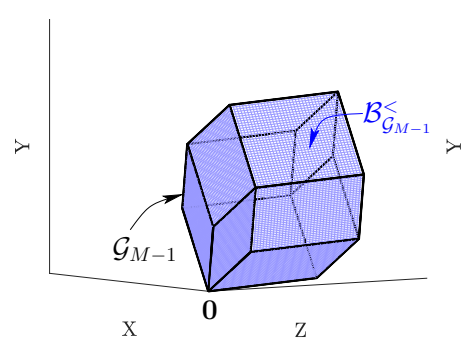

(a) $\mathcal{G}_{M-1}$ and $\mathbf{p}_{M}+\mathcal{B}_{\mathcal{G}_{M-1}}^{<}$

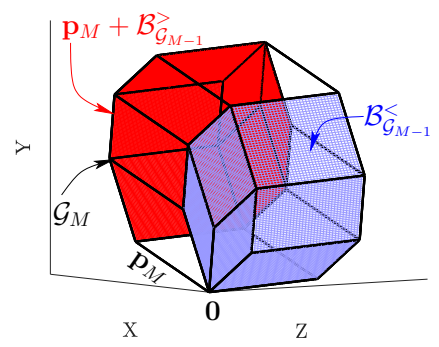

(c) $\mathcal{G}_{M}, \mathcal{B}_{\mathcal{G}_{M-1}}^{<}$, and $\mathbf{p}_{M}+\mathcal{B}_{\mathcal{G}_{M-1}}^{>}$

Fig. 9. The gamuts $\mathcal{G}_{M-1}$ and $\mathcal{G}_{M}$ and the relation between their boundaries: (a) $\mathcal{G}_{M-1}$ and $\mathcal{B}_{\mathcal{G}_{M-1}}^{<}$(blue); (b) $\mathcal{G}_{M-1}, \mathcal{B}_{\mathcal{G}_{M-1}}^{>}$(light red, with $\mathcal{B}_{\mathcal{G}_{M-1}}^{<}$ transparent with edges shown with dashed lines), and (c) $\mathcal{G}_{M}$ with $\mathcal{B}_{\mathcal{G}_{M-1}^{<}}^{<}$ (blue) and $\mathbf{p}_{M}+\mathcal{B}_{\mathcal{G}_{M-1}}^{>}$(red).

Now for $\mathbb{J} \in \mathfrak{C}^{2}(\langle M-1\rangle)$ consider the parallelepiped 
$\mathcal{P}\left(\mathbf{c}_{M}^{\mathbb{J}}, \mathbf{P}[\mathbb{J}, M]\right) \subset \mathcal{A}_{M}$ in (71). Via Lemma 1 , for each $\mathbf{t} \in \mathcal{P}\left(\mathbf{c}_{M}^{\mathbb{J}}, \mathbf{P}[\mathbb{J}, M]\right), \boldsymbol{\beta}=\mathbf{P}^{-1}[\mathbb{J}, M]\left(\mathbf{t}-\mathbf{c}_{M}^{\mathbb{J}}\right)$ is the unique vector in $[0,1]^{3}$ such that $\mathbf{t}=\mathbf{c}_{M}^{\mathbb{J}}+\mathbf{P}[\mathbb{J}, M] \boldsymbol{\beta}$. It follows that $\boldsymbol{\alpha}(\mathbf{t})=\boldsymbol{\alpha}_{M}^{\rrbracket}+\boldsymbol{\delta}^{\rrbracket, M}(\mathbf{t})$ represents a control vector

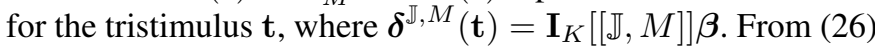
it can be seen that $\boldsymbol{\alpha}_{M}^{\mathbb{J}}$ is a control vector in $\mathcal{G}_{M-1}$ for $\mathbf{d}_{M-1}^{\mathbb{J}>}=\mathbf{c}_{M}^{\mathbb{J}}$ lying on the surface of $\mathcal{G}_{M-1}$, therefore, $\boldsymbol{\alpha}_{M}^{\mathbb{J}}$ is the unique control vector in $\mathcal{G}_{M-1}$ for $\mathbf{c}_{M}^{\mathbb{J}}$ (via the induction hypothesis). It follows that $\boldsymbol{\alpha}(\mathbf{t})=\boldsymbol{\alpha}_{M}^{\mathbb{J}}+\boldsymbol{\delta}^{\rrbracket, M}(\mathbf{t})$ is the unique unique control vector for $\mathbf{t}$ of the form the form $\delta_{l}^{(\mathbb{J}, M)}(\mathbf{t})=0$ for $l \notin[\mathbb{J}, M]$ and $0 \leq \delta_{l}^{(\mathbb{J}, M)}(\mathbf{t}) \leq 1$ for $l \in[\mathbb{J}, M]$. Also $\hat{\mathbf{t}}=\mathbf{t}-\beta_{3} \mathbf{p}_{M}$ is the unique vector of the form $\mathbf{t}-\beta \mathbf{p}_{M}$ lying on the surface of $\mathcal{G}_{M-1}$ and from the uniqueness of control values on the surface of $\mathcal{G}_{M-1}$ it then follows that if $\mathbf{t}$ lies on the surface of $\mathcal{G}_{M-1}$ then $\beta_{3}=0$ and if there exists another $\mathbb{J}^{\prime} \in \mathfrak{C}^{2}(\langle M-1\rangle)$ such that $\mathbf{t} \in \mathcal{P}\left(\mathbf{c}_{M}^{\mathbb{J}^{\prime}}, \mathbf{P}\left[\mathbb{J}^{\prime}, M\right]\right)$ then the control vectors for $\mathbf{t}$ in both the parallelepipeds are identical. Along with the induction hypothesis, the preceding conclusions imply that a $\mathrm{CCF} \mathscr{C}_{M}$ that is continuous and piece-wise linear over each of the parallelepiped partitions in the representation in (24) is obtained by defining, for $i=3,4, \ldots, M$ and for all $\mathbb{J} \in \mathfrak{C}^{2}([i-1])$,

$$
\mathscr{C}_{M}(\mathbf{t})=\boldsymbol{\alpha}_{i}^{\mathbb{J}}+\mathbf{I}_{M}[[\mathbb{J}, i]] \mathbf{P}^{-1}[\mathbb{J}, i]\left(\mathbf{t}-\mathbf{c}_{i}^{\mathbb{J}}\right),
$$

for all $\mathbf{t} \in \mathcal{P}\left(\mathbf{c}_{i}^{\mathbb{J}}, \mathbf{P}[\mathbb{J}, i]\right)$, where this CCF function is unique in the sense stated earlier. This completes the induction step for Clause 7 for Theorem 1.

Now we note that because the parallelepipeds in the righthand-side of (73) are essentially disjoint, the intersection of two parallelepipeds is contained within the boundary of both parallelepipeds. Combining this with the continuity and uniqueness of the CCF defined in (87) we can see that for any two parallelepipeds in (24) that have a non-empty intersection, the intersection is a proper face of both parallelepipeds, which completes the induction step for Clause 8 for Theorem 1 .

Next, we show that (31) and (32) uniquely associate two edges and a vertex to each of the facets, where the equations are interpreted as associating the sequence of two edges $\mathcal{E}\left(\mathbf{d}^{\mathbb{J}^{-}}, \mathbf{p}_{\mathbb{J}[1]}\right)$ and $\mathcal{E}\left(\mathbf{d}^{\mathbb{J}^{-}}+\mathbf{p}_{\mathbb{J}[1]}, \mathbf{p}_{\mathbb{J}[2]}\right)$ and the vertex $\mathbf{d}^{\mathbb{J}^{-}}+\mathbf{p}_{\mathbb{J}[1]}$ with the facet $\mathcal{F}\left(\mathbf{d}^{\mathbb{J}^{-}}, \mathbf{P}[\mathbb{J}]\right)$ and the sequence of two edges $\mathcal{E}\left(\mathbf{d}^{\mathbb{J}^{+}}, \mathbf{p}_{\mathbb{J}[2]}\right)$ and $\mathcal{E}\left(\mathbf{d}^{\mathbb{J}^{+}}+\mathbf{p}_{\mathbb{J}[2]}, \mathbf{p}_{\mathbb{J}[1]}\right)$ and the vertex $\mathbf{d}^{\mathbb{J}^{+}}+\mathbf{p}_{\mathbb{J}[2]}$ with the facet $\mathcal{F}\left(\mathbf{d}^{\mathbb{J}^{+}}, \mathbf{P}[\mathbb{J}]\right)$. Observing that $\mathbf{u}_{\mathbb{J}}$ is the direction pointing into and out-of the gamut $\mathcal{G}$ from the facets $\mathcal{F}\left(\mathbf{d}^{\mathbb{J}^{-}}, \mathbf{P}[\mathbb{J}]\right)$ and $\mathcal{F}\left(\mathbf{d}^{\mathbb{J}^{+}}, \mathbf{P}[\mathbb{J}]\right)$, respectively, we note that the sequence of edges associated with the facet is consistently and uniquely determined by the property that the cross-product between the primary spanning the first edge and the primary spanning the second edge is the direction pointing into the gamut $\mathcal{G}$ from the facet. Noting that the facets contain their associated vertices and edges, it follows that vertices and edges associated with disjoint facets are distinct and we therefore need only to consider distinct intersecting facets. Specifically, because $\tau^{\mathbb{J}}<\nu^{\mathbb{J}}$ the facets $\mathcal{F}\left(\mathbf{d}^{\mathbb{J}^{\prime}-}, \mathbf{P}[\mathbb{J}]\right)$ and $\mathcal{F}\left(\mathbf{d}^{\mathbb{J}^{\prime}+}, \mathbf{P}[\mathbb{J}]\right)$ are disjoint and we only need consider distinct facets $\mathcal{F}(\mathbf{o}, \mathbf{P}[\mathbb{J}])$ and $\mathcal{F}\left(\mathbf{o}^{\prime}, \mathbf{P}\left[\mathbb{J}^{\prime}\right]\right)$, where $\mathbb{J}, \mathbb{J}^{\prime} \in \mathfrak{C}^{2}(\langle K\rangle), \mathbb{J}^{\prime} \neq \mathbb{J}, \mathbf{o} \in\left\{\mathbf{d}^{\mathbb{J}^{-}}, \mathbf{d}^{\mathbb{J}^{+}}\right\}$ and $\mathbf{o}^{\prime} \in\left\{\mathbf{d}^{\mathbb{J}^{\prime}-}, \mathbf{d}^{\mathbb{J}^{\prime}+}\right\}$. As the intersection of two different twodimensional faces of a polytope is another face of lower dimensionality, the intersection $\mathcal{A}=\mathcal{F}(\mathbf{o}, \mathbf{P}[\mathbb{J}]) \cap \mathcal{F}\left(\mathbf{o}^{\prime}, \mathbf{P}\left[\mathbb{J}^{\prime}\right]\right)$ is either a vertex $\mathbf{o}_{v}$ or an edge $\mathcal{E}\left(\mathbf{o}_{e}, \mathbf{p}_{m}\right)$, with origin $\mathbf{o}_{e}$ and spanned by $\mathbf{p}_{m}$ where $m=\mathbb{J} \cap \mathbb{J}^{\prime}$.

Assume first that $\mathcal{A}=\mathcal{E}\left(\mathbf{o}_{e}, \mathbf{p}_{m}\right)$. We denote $j=\mathbb{J} \backslash m$ and $j^{\prime}=\mathbb{J}^{\prime} \backslash m$ and use the convenient short hand labels $\mathcal{F}_{j}=\mathcal{F}(\mathbf{o}, \mathbf{P}[\mathbb{J}]), \mathcal{F}_{j^{\prime}}=\mathcal{F}\left(\mathbf{o}^{\prime}, \mathbf{P}\left[\mathbb{J}^{\prime}\right]\right), \mathbf{o}_{j}=\mathbf{o}, \mathbf{o}_{j^{\prime}}=\mathbf{o}^{\prime}$ for the facets and their origins. Assume wlog (otherwise, simply interchange $\mathbb{J}$ and $\left.\mathbb{J}^{\prime}\right)$ that $\operatorname{sgn}\left(\operatorname{det}\left(\left[\mathbf{p}_{m}, \delta_{j} \mathbf{p}_{j}, \delta_{j^{\prime}} \mathbf{p}_{j^{\prime}}\right]\right)\right)=1$, so that $\mathbf{p}_{m}, \delta_{j} \mathbf{p}_{j}, \delta_{j^{\prime}} \mathbf{p}_{j^{\prime}}$ forms a positively oriented basis for $\mathbb{R}^{3}$, where, for $q=j, j^{\prime}, \delta_{q} \mathbf{p}_{q}$ is the feasible direction along the facet $\mathcal{F}_{q}$ from the edge $\mathcal{E}\left(\mathbf{o}_{e}, \mathbf{p}_{m}\right)$, with $\delta_{q} \in\{ \pm 1\}$ determined by the facet $\mathcal{F}_{q}$ via the relation $\mathbf{o}_{q}=\mathbf{o}_{e}-\left(\left(\delta_{q}-1\right) / 2\right) \mathbf{p}_{q}$. From the convexity of the gamut $\mathcal{G}$ and our chosen orientation, it then follows that $\left(\delta_{j^{\prime}} \mathbf{p}_{j^{\prime}} \otimes \mathbf{p}_{m}\right)$ points into the gamut $\mathcal{G}$ from the facet $\mathcal{F}_{j^{\prime}}$. Now if $\delta_{j^{\prime}}=1,\left(\mathbf{p}_{j^{\prime}} \otimes \mathbf{p}_{m}\right)$ points into the gamut $\mathcal{G}$ from the facet $\mathcal{F}_{j^{\prime}}, \mathbf{o}_{j^{\prime}}=\mathbf{o}_{e}$, and the edges $\mathcal{E}\left(\mathbf{o}_{j^{\prime}}, \mathbf{p}_{j^{\prime}}\right)=\mathcal{E}\left(\mathbf{o}_{e}, \mathbf{p}_{j^{\prime}}\right)$ and $\mathcal{E}\left(\mathbf{o}_{j^{\prime}}+\mathbf{p}_{j^{\prime}}, \mathbf{p}_{m}\right)=\mathcal{E}\left(\mathbf{o}_{e}+\mathbf{p}_{j^{\prime}}, \mathbf{p}_{m}\right)$ and the vertex $\mathbf{o}_{j^{\prime}}+\mathbf{p}_{j^{\prime}}=\mathbf{o}_{e}+\mathbf{p}_{j^{\prime}}$ are associated with the facet $\mathcal{F}_{j^{\prime}}$. On the other hand, if $\delta_{j^{\prime}}=-1,\left(\mathbf{p}_{m} \otimes \mathbf{p}_{j^{\prime}}\right)$ points into the gamut $\mathcal{G}$ from the facet $\mathcal{F}_{j^{\prime}}, \mathbf{o}_{j^{\prime}}=\mathbf{o}_{e}-\mathbf{p}_{j^{\prime}}$, and the edges $\mathcal{E}\left(\mathbf{o}_{j^{\prime}}, \mathbf{p}_{m}\right)=\mathcal{E}\left(\mathbf{o}_{e}-\mathbf{p}_{j^{\prime}}, \mathbf{p}_{m}\right)$ and $\mathcal{E}\left(\mathbf{o}_{j^{\prime}}+\mathbf{p}_{m}, \mathbf{p}_{m}\right)=$ $\mathcal{E}\left(\mathbf{o}_{e}-\mathbf{p}_{j^{\prime}}+\mathbf{p}_{m}, \mathbf{p}_{j^{\prime}}\right)$ and the vertex $\mathbf{o}_{j^{\prime}}+\mathbf{p}_{m}=\mathbf{o}_{e}-\mathbf{p}_{j^{\prime}}+\mathbf{p}_{m}$ are associated with the facet $\mathcal{F}_{j^{\prime}}$. In both cases, we see that the edge $\mathcal{E}\left(\mathbf{o}_{e}, \mathbf{p}_{m}\right)$ that is shared between the facets $\mathcal{F}_{j}$ and $\mathcal{F}_{j^{\prime}}$ is not associated with the $\mathcal{F}_{j^{\prime}}$ and that the vertex associated with the facet $\mathcal{F}_{j^{\prime}}$ is not contained in the facet $\mathcal{F}_{j}$ (and analogous arguments demonstrate that in both cases the edge $\mathcal{E}\left(\mathbf{o}_{e}, \mathbf{p}_{m}\right)$ is associated with the facet $\mathcal{F}_{j}$ ) It therefore follows that the edge and vertex associations for two facets sharing a common edge cannot conflict and are unique. Figure 10 provides an illustration of the situation that provides geometric intuition for the preceding algebraic arguments and Table VI lists the different possible cases for the edge and vertex associations.

Next consider the case when the intersection $\mathcal{A}=\left\{\mathbf{o}_{v}\right\}$ is a vertex of both $\mathcal{F}_{\mathbb{J}}=\mathcal{F}(\mathbf{o}, \mathbf{P}[\mathbb{J}])$ and $\mathcal{F}_{\mathbb{J}^{\prime}}=\mathcal{F}\left(\mathbf{o}^{\prime}, \mathbf{P}\left[\mathbb{J}^{\prime}\right]\right)$. From the uniqueness of the control values for a vertex and the linear independence for any subset of three primaries, it follows that $\mathbb{J} \cap \mathbb{J}^{\prime}=\phi$. Therefore, the edges associated with the intersecting facets are clearly different. Thus, we only need to show that the associated vertices are different as well. Specifically, we show that if $\mathbf{o}_{v}$ is associated with both of the intersecting facets, then one of the four primaries in $\mathbf{P}\left[\mathbb{J}, \mathbb{J}^{\prime}\right]$ is the linear combination of the remaining three using exclusively negative coefficients ${ }^{14}$, which, being impossible for physically meaningful primaries, yields our desired result by contraposition. Now assume that (32) associates the shared vertex $\mathbf{o}_{v}$ with both facets $\mathcal{F}_{\mathbb{J}}$ and $\mathcal{F}_{\mathbb{J}^{\prime}}$. From (32) we see that $\mathbf{o}_{v} \neq \mathbf{o}$ and $\mathbf{o}_{v} \neq \mathbf{o}^{\prime}$. Let $\mathbf{p}_{i}, \mathbf{p}_{j}$ denote the sequence of spanning

\footnotetext{
${ }^{14}$ Instead of introducing additional notation, we continue to refer to the vectors spanning the facets as primaries in the rest of this paragraph, even though they may include negative values, which is not possible for physically meaningful primary tristimuli.
} 

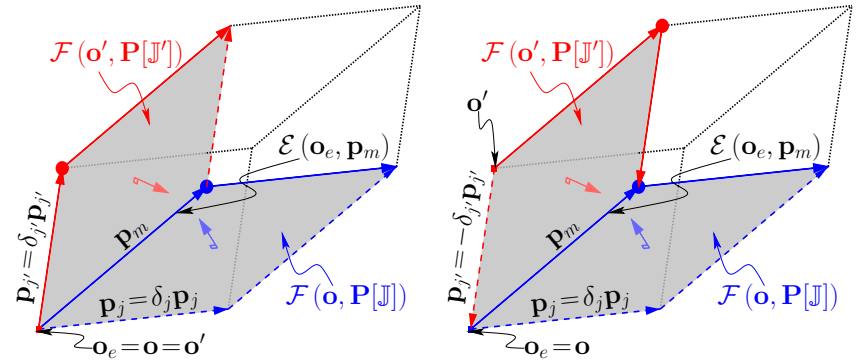

(a) $\delta_{j}=1, \delta_{j^{\prime}}=1$

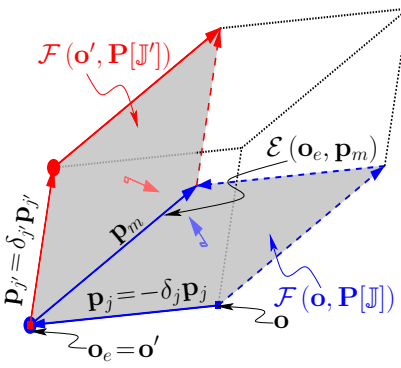

(c) $\delta_{j}=-1, \delta_{j^{\prime}}=1$ (b) $\delta_{j}=1, \delta_{j^{\prime}}=-1$

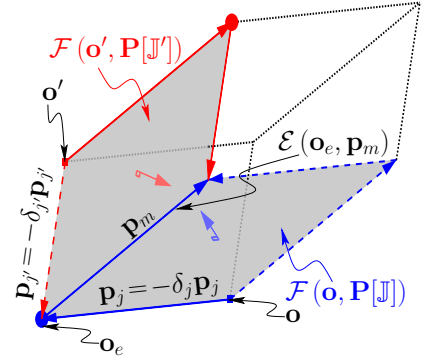

(d) $\delta_{j}=-1, \delta_{j^{\prime}}=-1$

Fig. 10. Edge and vertex associations predicated by (31) and (32) for a pair of facets $\mathcal{F}_{j}=\mathcal{F}\left(\mathbf{o}, \mathbf{P}[\mathbb{J}]\right.$ ) (gray shade with blue edges) and $\mathcal{F}_{j^{\prime}}=$ $\mathcal{F}\left(\mathbf{o}^{\prime}, \mathbf{P}\left[\mathbb{J}^{\prime}\right]\right)$ (gray shade with red edges) intersecting in a common edge $\mathcal{E}\left(\mathbf{o}_{e}, \mathbf{p}_{m}\right)$ that has origin $\mathbf{o}_{e}$ and is spanned by $\mathbf{p}_{m}$, where $m=\mathbb{I} \cap \mathbb{J}^{\prime}$, $j=\mathbb{J} \backslash m$ and $j^{\prime}=\mathbb{J}^{\prime} \backslash m$. The direction pointing into the gamut $\mathcal{G}$ from the facet is identified by, respectively, the blue and red normal vectors placed at the center of the facets $\mathcal{F}(\mathbf{o}, \mathbf{P}[\mathbb{J}])$ and $\mathcal{F}\left(\mathbf{o}^{\prime}, \mathbf{P}\left[\mathbb{J}^{\prime}\right]\right)$. See text for additional description and definitions of $\delta_{j}, \delta_{j^{\prime}}$.

primaries associated with the $\mathcal{F}_{\mathbb{J}}$ such that $\mathbf{o}_{v}=\mathbf{o}+\mathbf{p}_{i}$ and let $\mathbf{p}_{l}, \mathbf{p}_{m}$ denote the sequence of primaries associated with the facet $\mathcal{F}_{\mathbb{J}^{\prime}}$ such that $\mathbf{o}_{v}=\mathbf{o}+\mathbf{p}_{l}$. Then, the normals $\mathbf{n}_{\mathbb{J}}=\left(\mathbf{p}_{i} \otimes \mathbf{p}_{j}\right)$ and $\mathbf{n}_{\mathbb{J}^{\prime}}=\left(\mathbf{p}_{l} \otimes \mathbf{p}_{m}\right)$ point into the gamut $\mathcal{G}$ from the facets $\mathcal{F}_{\mathbb{J}}$ and $\mathcal{F}_{\mathbb{J}}$ and the minima of the functions $\mathbf{n}_{\mathbb{J}}^{T} \mathbf{t}, \mathbf{n}_{\mathbb{J}}^{T} \mathbf{t}$ over $\mathbf{t} \in \mathcal{G}$ are achieved precisely over the sets $\mathcal{F}_{\mathbb{J}}$, $\mathcal{F}_{\mathbb{J}^{\prime}}$, respectively. Noting that the vertex $\mathbf{o}_{v}$ is the intersection of the sequence of edges associated with its facet, we see that for $\alpha \in[0,1], \mathbf{o}_{v}-\alpha \mathbf{p}_{q} \in \mathcal{G}$ for $q=i, l$ and $\mathbf{o}_{v}+\alpha \mathbf{p}_{q} \in \mathcal{G}$ for $q=j, m$. It follows that $\mathbf{n}_{\mathbb{J}}^{T} \mathbf{p}_{m}=\operatorname{det}\left(\left[\mathbf{p}_{i}, \mathbf{p}_{j}, \mathbf{p}_{m}\right]\right)>0>$ $\mathbf{n}_{\mathbb{J}}^{T} \mathbf{p}_{l}=\operatorname{det}\left(\left[\mathbf{p}_{i}, \mathbf{p}_{j}, \mathbf{p}_{l}\right]\right)$ and $\mathbf{n}_{\mathbb{J}}^{T} \mathbf{p}_{j}=\operatorname{det}\left(\left[\mathbf{p}_{l}, \mathbf{p}_{m}, \mathbf{p}_{j}\right]\right)>$ $0>\mathbf{n}_{\mathbb{J}^{\prime}}^{T} \mathbf{p}_{i}=\operatorname{det}\left(\left[\mathbf{p}_{l}, \mathbf{p}_{m}, \mathbf{p}_{i}\right]\right)$. From these inequalities, we can conclude that the primaries $\mathbf{p}_{i}, \mathbf{p}_{j}, \mathbf{p}_{m}$ form a positively oriented basis for $\mathbb{R}^{3}$. Next, we express the primary $\mathbf{p}_{l}$ in terms of this basis as $\mathbf{p}_{l}=\sum_{q=i, j, m} \beta_{q} \mathbf{p}_{q}$, where $\beta_{q} \in \mathbb{R}^{3}$. Now for this (in general, oblique) positively-oriented basis representation, we have (see Clause 5 for Lemma 1)

$$
\begin{gathered}
\operatorname{sgn}\left(\beta_{i}\right)=\operatorname{sgn}\left(\left(\mathbf{p}_{j} \otimes \mathbf{p}_{m}\right)^{T} \mathbf{p}_{l}\right)=\operatorname{sgn}\left(\operatorname{det}\left(\left[\mathbf{p}_{j}, \mathbf{p}_{m}, \mathbf{p}_{l}\right]\right)\right)=-1 \\
\operatorname{sgn}\left(\beta_{j}\right)=\operatorname{sgn}\left(\left(\mathbf{p}_{m} \otimes \mathbf{p}_{i}\right)^{T} \mathbf{p}_{l}\right)=\operatorname{sgn}\left(\operatorname{det}\left(\left[\mathbf{p}_{m}, \mathbf{p}_{i}, \mathbf{p}_{l}\right]\right)\right)=-1 \\
\operatorname{sgn}\left(\beta_{m}\right)=\operatorname{sgn}\left(\left(\mathbf{p}_{i} \otimes \mathbf{p}_{j}\right)^{T} \mathbf{p}_{l}\right)=\operatorname{sgn}\left(\operatorname{det}\left(\left[\mathbf{p}_{i}, \mathbf{p}_{j}, \mathbf{p}_{l}\right]\right)\right)=-1
\end{gathered}
$$

Therefore, $\mathbf{p}_{l}$ is the linear combination of $\mathbf{p}_{i}, \mathbf{p}_{j}, \mathbf{p}_{m}$ using exclusively negative coefficients. Figure 11 provides an illustration of the situation that provides geometric intuition for the preceding algebraic arguments. Now, by contraposition, it follows that for physically meaningful primaries that have only non-negative values, the enumerated vertices in (32) cannot

be simultaneously associated with two different intersecting facets, and are therefore unique.

Noting that the vertices corresponding to display black $\mathbf{t}_{\mathbf{0}}$ and display white $\mathbf{t}_{\mathbf{1}}$ are not associated with any facet, but are explicitly included in (32), we can conclude that (32) uniquely enumerates $M(M-1)+2$ vertices of $\mathcal{G}_{M}$ and (31) uniquely enumerates $2 M(M-1)$ edges for $\mathcal{G}_{M}$. Because, we we have these values as the number of distinct edges and vertices, respectively, in $\mathcal{G}_{M}$, we have hereby completed the induction step for Clauses 4 and 5 in Theorem 1 .

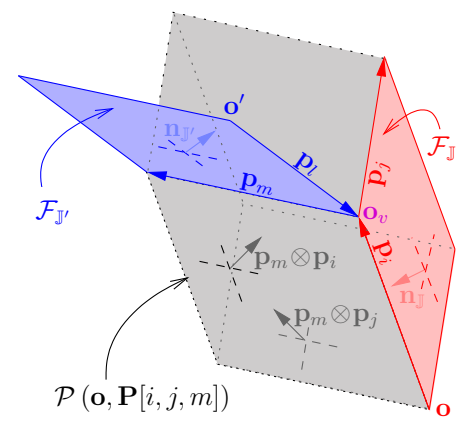

Fig. 11. Intersecting facets with the same associated vertex. A pair of gamut facets $\mathcal{F}_{\mathbb{J}}=\mathcal{F}(\mathbf{o}, \mathbf{P}[\mathbb{J}])$ (red) and $\mathcal{F}_{\mathbb{J}^{\prime}}=\mathcal{F}\left(\mathbf{o}^{\prime}, \mathbf{P}\left[\mathbb{J}^{\prime}\right]\right)$ (blue), with $\mathbb{J} \cap \mathbb{J}^{\prime}=\phi$, intersect in a common vertex $\mathbf{o}_{v}$ (magenta), which is assumed to be associated with both facets. The primary pairs $\mathbf{p}_{i}, \mathbf{p}_{j}$ and $\mathbf{p}_{l}, \mathbf{p}_{m}$ span the sequence of associated edges for the facets $\mathcal{F}_{\mathbb{J}}$ and $\mathcal{F}_{\mathbb{J}^{\prime}}$, respectively, so the facet normals $\mathbf{n}_{\mathbb{J}}$ and $\mathbf{n}_{\mathbb{J}^{\prime}}$ point into the gamut from the facets. The primaries $\mathbf{p}_{i}, \mathbf{p}_{j}, \mathbf{p}_{m}$ form a positive oriented basis, and $\mathbf{p}_{l}$ is the linear combination of $\mathbf{p}_{i}, \mathbf{p}_{j}, \mathbf{p}_{m}$ using exclusively negative coefficients. The parallelepiped $\mathcal{P}\left(\mathbf{o},\left[\mathbf{p}_{i}, \mathbf{p}_{j}, \mathbf{p}_{m}\right]\right) \subset \mathcal{G}$ is shown in gray to help with visualization of the situation in three dimensions.

\section{APPENDIX C \\ SPECIFICATIONS OF PRIMARY SYSTEMS USED IN EXAMPLES}

Table III lists the tristimulus values corresponding to the columns of the primary matrices used as examples in the paper. The primary systems $\mathbf{P}_{\mathscr{V}}^{(K)}$, with $K=3,4,5$, and 6 , maximize volume in the CIELUV space, under a total power constraint, and are obtained following the methodology proposed in [23]. The four-primary system $\mathbf{P}_{w}^{(4)}$ and the fiveprimary system $\mathbf{P}_{w}^{(5)}$ are obtained as extensions of the optimal systems $\mathbf{P}_{\mathscr{V}}^{(3)}$ and $\mathbf{P}_{\mathscr{V}}^{(4)}$, respectively, by adding a primary whose chromaticity matches the display white chromaticity; specifically, $\mathbf{P}_{w}^{(4)}=\left[(2 / 3) \mathbf{P}_{\mathscr{V}}^{(3)} \mid(1 / 3) \mathbf{P}_{\mathscr{V}}^{(3)} \mathbf{1}\right]$ and $\mathbf{P}_{w}^{(5)}=$ $\left[(3 / 4) \mathbf{P}_{\mathscr{V}}^{(4)} \mid(1 / 4) \mathbf{P}_{\mathscr{V}}^{(4)} \mathbf{1}\right]$

\section{APPENDIX D}

\section{AdDitional Tristimulus GAMUt VisUALIZATIONS}

Figure 12 illustrates the tristimulus gamuts $\mathcal{G}$ for the 5 primary system $\mathbf{P}_{w}^{(5)}$ and the six primary system $\mathbf{P}_{\mathscr{V}}^{(6)}$ (with $\mathbf{t}_{\mathbf{0}}=\mathbf{0}$ in all cases). The edges and vertices of the gamut are colored to indicate the associations as per Clauses 4 and 5 of Theorem 1 using a convention identical to that used for Fig. 2. 


\begin{tabular}{|c|c|c|c|c|c|c|c|}
\hline & & $\mathbf{p}_{1}$ & $\mathbf{p}_{2}$ & $\mathbf{p}_{3}$ & $\mathbf{p}_{4}$ & $\mathbf{p}_{5}$ & $\mathbf{p}_{6}$ \\
\hline \multirow{3}{*}{$\mathbf{P}_{\mathscr{V}}^{(3)}$} & $X$ & 20.15 & 4.46 & 70.56 & & & \\
\hline & Y & 0.65 & 73.70 & 25.65 & & & \\
\hline & $\mathrm{Z}$ & 97.21 & 11.77 & 0.00 & & & \\
\hline \multirow{3}{*}{$\mathbf{P}_{\mathscr{V}}^{(4)}$} & $\mathrm{X}$ & 16.88 & 0.78 & 5.67 & 71.82 & & \\
\hline & Y & 0.49 & 19.81 & 53.59 & 26.11 & & \\
\hline & Z & 80.98 & 22.86 & 5.17 & 0.00 & & \\
\hline \multirow{4}{*}{$\mathbf{P}_{\mathscr{V}}^{(5)}$} & $\mathrm{X}$ & 15.15 & 2.24 & 0.67 & 6.32 & 70.77 & \\
\hline & Y & 0.42 & 7.66 & 31.87 & 34.28 & 25.77 & \\
\hline & Z & 72.24 & 25.03 & 9.96 & 1.79 & 0.00 & \\
\hline & $\mathrm{X}$ & 14.56 & 2.48 & 0.14 & 2.88 & 5.24 & 69.87 \\
\hline \multirow{2}{*}{$\mathbf{P}_{\mathscr{V}}^{(6)}$} & Y & 0.42 & 6.57 & 18.63 & 34.20 & 14.79 & 25.40 \\
\hline & Z & 69.62 & 25.31 & 9.70 & 4.11 & 0.22 & 0.00 \\
\hline \multirow{4}{*}{$\mathbf{P}_{w}^{(4)}$} & $X$ & 13.43 & 2.97 & 47.04 & 31.72 & & \\
\hline & $\mathrm{Y}$ & 0.43 & 49.13 & 17.10 & 33.33 & & \\
\hline & Z & 64.81 & 7.85 & 0.00 & 36.33 & & \\
\hline & $\mathrm{X}$ & 12.66 & 0.58 & 4.25 & 53.87 & 23.79 & \\
\hline \multirow{2}{*}{$\mathbf{P}_{w}^{(5)}$} & Y & 0.37 & 14.86 & 40.19 & 19.58 & 25.00 & \\
\hline & Z & 60.73 & 17.15 & 3.88 & 0.00 & 27.25 & \\
\hline
\end{tabular}

CIE XYZ TRISTIMULI OF THE PRIMARIES FOR THE MULTIPRIMARY SYSTEMS USED FOR THE EXAMPLES IN THE PAPER.

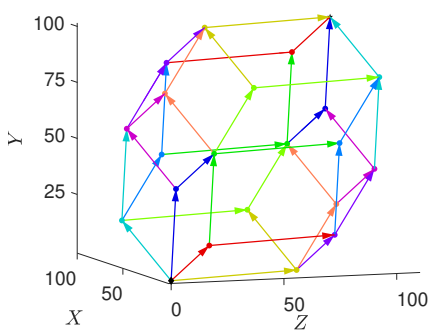

(a) $K=5$

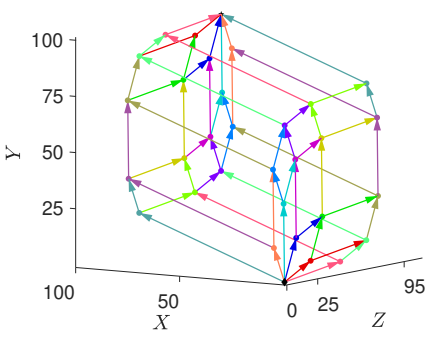

(b) $K=6$
Fig. 12. The tristimulus gamuts $\mathcal{G}$ for the $K=5$ and 6 primary CIELUV gamut volume maximizing designs $\mathbf{P}_{w}^{(5)}$, and $\mathbf{P}_{\mathscr{V}}^{(6)}$. In each case, the region inside the wireframe corresponds to the gamut and the edges and vertices are colored to indicate the associations as per Clauses 4 and 5 of Theorem 1 (see text).

\section{APPENDIX E}

\section{EXAMPles of PROgRESSIVE GAMUT TILINGS}

In this section, we present the different progressive-byprimary tilings of the gamut of the $K=5$ primary system $\mathbf{P}_{w}^{(5)}$ produced by Algorithm 1. While the different progressive-byprimary tilings are produced from different permutations of the primaries in $\mathbf{P}_{w}^{(5)}$, the fact that the first parallelepiped in the tiling is defined by the first three primaries in $\mathbf{P}_{w}^{(5)}$ indicates that Algorithm 1 can produce a maximum of $\left(\begin{array}{c}K \\ 3\end{array}\right)(K-3)$ ! = $K ! / 3=20$ different tilings. For the primary system $\mathbf{P}_{w}^{(5)}$, Algorithm 1 actually produces 8 different tilings shown in Fig. 13, with the list of the permutations of $\mathbf{P}_{w}^{(5)}$ producing each gamut tiling shown in Table IV. To allow the distinct parallelepipeds in the tilings to be seen, the centers of the parallelepipeds have been displaced radially outward with respect to the center of the gamut (by scaling the original displacements by a factor of 1.8) and the axes in Fig. 13 are therefore labeled as $X^{\prime}, Y^{\prime}, Z^{\prime}$.

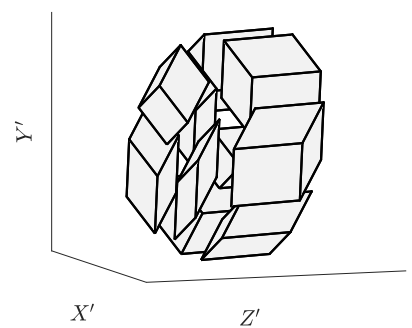

(a) Tiling 1

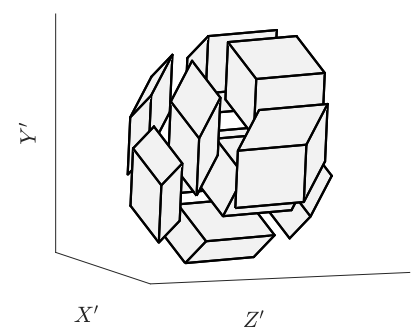

(c) Tiling 3

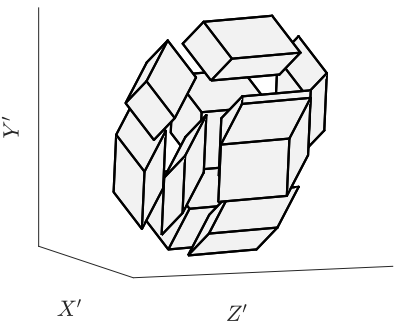

(e) Tiling 5

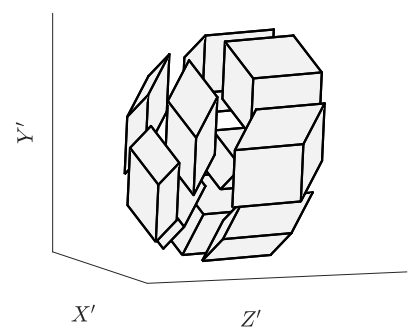

(g) Tiling 7

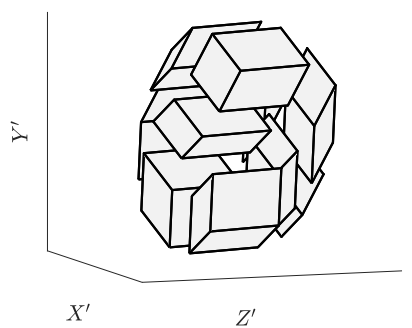

(b) Tiling 2

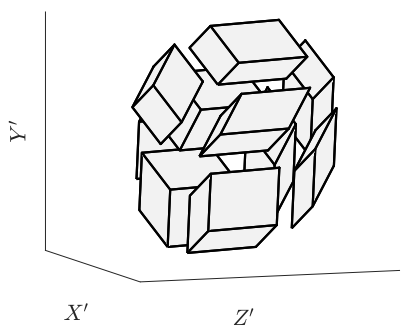

(d) Tiling 4

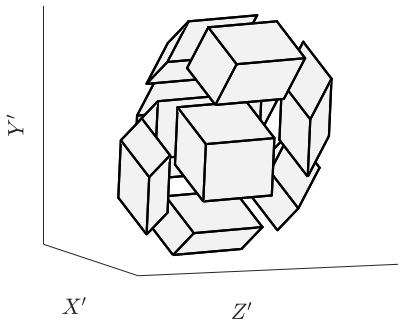

(f) Tiling 6

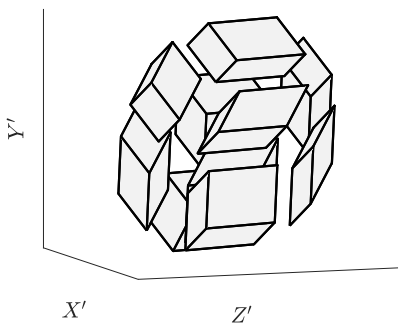

(h) Tiling 8
Fig. 13. The progressive-by-primary tilings of the gamut of the $K=5$ primary system $\mathbf{P}_{w}^{(5)}$, produced by Algorithm 1 with the columns of $\mathbf{P}_{w}^{(5)}$ permuted in different orders. Table IV lists the permutations corresponding to each of the tilings.

\section{APPENDIX F}

\section{UNIQUENESS OF VERTEX ENUMERATION IS INAPPLICABLE FOR GENERAL ZONOTOPES}

Let $\mathbf{v}$ be any vector in $\mathbb{R}^{3}$ and $\mathbf{A} \in \mathbb{R}^{3 \times K}$ be a matrix, any three of whose columns are linearly independent. Consider the associated three dimensional zonotope

$$
\mathcal{A}(\mathbf{v}, \mathbf{A})=\left\{\mathbf{A} \boldsymbol{\alpha}+\mathbf{v} \mid \boldsymbol{\alpha} \in[0,1]^{K}\right\},
$$

that exactly mirrors our definition of the multiprimary gamut in (4), with the exception that while entries in $\mathbf{P}$ were constrained by physics to be nonnegative, no such constraint applies to the entries in A. Then, as already noted, except for Clause 5, all other clauses of Theorem 1, also apply to the representation of the three-dimensional zonotope in (88). 


\begin{tabular}{|c|c|c|c|}
\hline Tiling & Figure & Primary Permuta & ation \\
\hline 1 & Fig. 13(a) & $\begin{array}{llll}\mathbf{p}_{2} & \mathbf{p}_{3} & \mathbf{p}_{5} & \mathbf{p}_{4} \\
\mathbf{p}_{3} & \mathbf{p}_{4} & \mathbf{p}_{5} & \mathbf{p}_{2} \\
\end{array}$ & $\begin{array}{l}\mathbf{p}_{1} \\
\mathbf{p}_{1}\end{array}$ \\
\hline 2 & Fig. 13(b) & $\begin{array}{llll}\mathbf{p}_{1} & \mathbf{p}_{2} & \mathbf{p}_{3} & \mathbf{p}_{4} \\
\mathbf{p}_{1} & \mathbf{p}_{3} & \mathbf{p}_{4} & \mathbf{p}_{2} \\
\end{array}$ & \\
\hline 3 & Fig. 13(c) & $\begin{array}{llllllllllll}\mathbf{p}_{1} & \mathbf{p}_{2} & \mathbf{p}_{4} & \mathbf{p}_{5}\end{array}$ & $\mathbf{p}_{3}$ \\
\hline 4 & Fig. 13(d) & $\mathbf{p}_{1} \mathbf{p}_{3} \mathbf{p}_{4} \mathbf{p}_{5}$ & $\mathbf{p}_{2}$ \\
\hline 5 & Fig. 13(e) & $\begin{array}{llll}\mathbf{p}_{1} & \mathbf{p}_{2} & \mathbf{p}_{5} & \mathbf{p}_{3} \\
\mathbf{p}_{2} & \mathbf{p}_{3} & \mathbf{p}_{5} & \mathbf{p}_{1} \\
\end{array}$ & \\
\hline 6 & Fig. 13(f) & $\begin{array}{llll}\mathbf{p}_{1} & \mathbf{p}_{2} & \mathbf{p}_{4} & \mathbf{p}_{3} \\
\mathbf{p}_{2} & \mathbf{p}_{3} & \mathbf{p}_{4} & \mathbf{p}_{1}\end{array}$ & \\
\hline 7 & Fig. 13(g) & $\begin{array}{llll}\mathbf{p}_{2} & \mathbf{p}_{3} & \mathbf{p}_{4} & \mathbf{p}_{5} \\
\mathbf{p}_{1} & \mathbf{p}_{2} & \mathbf{p}_{5} & \mathbf{p}_{4} \\
\mathbf{p}_{1} & \mathbf{p}_{4} & \mathbf{p}_{5} & \mathbf{p}_{2} \\
\mathbf{p}_{2} & \mathbf{p}_{4} & \mathbf{p}_{5} & \mathbf{p}_{3} \\
\mathbf{p}_{2} & \mathbf{p}_{4} & \mathbf{p}_{5} & \mathbf{p}_{1} \\
\end{array}$ & $\begin{array}{l}\mathbf{p}_{1} \\
\mathbf{p}_{3} \\
\mathbf{p}_{3} \\
\mathbf{p}_{1} \\
\mathbf{p}_{3} \\
\end{array}$ \\
\hline 8 & Fig. 13(h) & $\begin{array}{llll}\mathbf{p}_{1} & \mathbf{p}_{2} & \mathbf{p}_{3} & \mathbf{p}_{5} \\
\mathbf{p}_{1} & \mathbf{p}_{3} & \mathbf{p}_{5} & \mathbf{p}_{4} \\
\mathbf{p}_{1} & \mathbf{p}_{3} & \mathbf{p}_{5} & \mathbf{p}_{2} \\
\mathbf{p}_{1} & \mathbf{p}_{4} & \mathbf{p}_{5} & \mathbf{p}_{3} \\
\mathbf{p}_{3} & \mathbf{p}_{4} & \mathbf{p}_{5} & \mathbf{p}_{1}\end{array}$ & $\begin{array}{l}\mathbf{p}_{4} \\
\mathbf{p}_{2} \\
\mathbf{p}_{4} \\
\mathbf{p}_{2} \\
\mathbf{p}_{2}\end{array}$ \\
\hline
\end{tabular}

PRIMARY PERMUTATIONS PRODUCING THE DIFFERENT PROGRESSIVE-BY-PRIMARY TILINGS OF THE GAMUT OF THE $K=5$ PRIMARY SYSTEM $\mathbf{P}_{w}^{(5)}$.

From the proof for Theorem 1, we see that the uniqueness of the vertices enumerated in (32) and the validity of Clause 5 holds provided no column of $\mathbf{A}$ is a linear combination of three other columns of $\mathbf{A}$ using exclusively negative coefficients. Because this condition directly holds for primary matrices $\mathbf{P}$ with nonnegative entries, Theorem 1 holds in general for any multiprimary display gamut. Here we provide a example demonstrating failure of the uniqueness of the vertices enumerated in (32) for a three-dimensional zonotope obtained with a matrix $\mathbf{A}$ one of whose columns is a linear combination of three other columns of $\mathbf{A}$ using exclusively negative coefficients. Specifically consider the $3 \times 4$ matrix

$$
\mathbf{A} \stackrel{\text { def }}{=}\left[\begin{array}{llll}
\mathbf{a}_{1} & \mathbf{a}_{2} & \mathbf{a}_{3} & \mathbf{a}_{4}
\end{array}\right]=\left[\begin{array}{cccc}
1 & 0 & 0 & -0.5 \\
0 & 1 & 0 & -0.5 \\
0 & 0 & 1 & -0.5
\end{array}\right],
$$

where it can be noted that $\mathbf{a}_{4}=-0.5\left(\mathbf{a}_{1}+\mathbf{a}_{2}+\mathbf{a}_{3}\right)$. The corresponding three dimensional zonotope $\mathcal{A}(\mathbf{0}, \mathbf{A})$ is shown in Fig 14, where the two edges of the same color in a facet indicate the edges associated with the facet through (31) and the vertex located at the intersection of these edges is the vertex that (32) associates with the same facet. By examining, Fig 14 we can see that the vertex $\mathbf{t}_{v}=[-0.5,0.5,-0.5]^{T}$ is associated with two different facets (corresponding to the facets that have two brown and two blue colored edges). Thus the enumeration of the vertices predicated by (32) in Theorem 1 is not unique for the zonotope $\mathcal{A}(\mathbf{0}, \mathbf{A})$. From Fig. 14, we also note that for the zonotope $\mathcal{A}(\mathbf{0}, \mathbf{A})$, the expressions in (32) fail to enumerate several of the vertices (vertices not marked by circles in Fig. 14) and incorrectly identify the points $\mathbf{v}=\mathbf{0}$ (marked by the diamond in Fig. 14) and $\mathbf{v}+\sum_{k=1}^{4} \mathbf{a}_{k}$ (marked by the star in Fig. 14) as vertices. We note that our results do not establish that the uniqueness of the vertex associations in (32) fails whenever one of the columns of the matrix $\mathbf{A}$ is a linear combination of three other columns of A using exclusively negative coefficients, though computational exploration suggests this is the case.

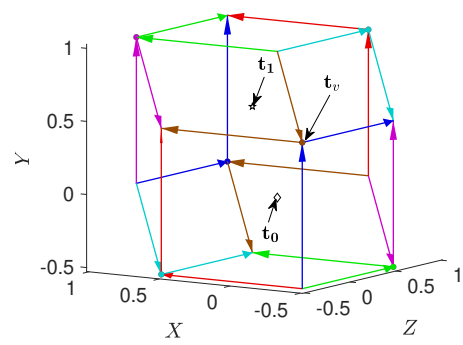

Fig. 14. Example illustrating the three dimensional zonotope $\mathcal{A}(\mathbf{0}, \mathbf{A})$ where $\mathbf{A}$ is defined in (89) for which the enumeration of vertices by (32) in Theorem 1 fails. See text for description.

\section{APPENDIX G}

\section{APPLICATION OF THEOREM 1 FOR POLAR ZONOHEDRA}

A polar zonohedra is a zonotope $\mathcal{A}$ in $\mathbb{R}^{3}$ resulting from the mapping of $[0,1]^{K}, K \geq 3$ by a $3 \times K$ matrix A whose columns are vectors starting at the apex of a circular cone and ending at points located on the base evenly spaced in angle [35]. Even though the matrix $\mathbf{A}$ that generates a polar zonohedron can contain negative entries (unlike multiprimary display matrices), as noted in Section VI Theorem 1, applies in its entirety to polar zonohedra and can therefore be used to directly obtain geometric representations of polar zonohedra. We illustrate this application in this section.

Using a circular cone of radius $r$, height $R$ and apex at the origin 0 , a polar zonohedra for $K \geq 3$ is obtained by the matrix denoted by $\mathbf{A}_{\circ}^{(K)}$ with column vectors $\mathbf{a}_{k}=$ $[r \cos (2 \pi k / K), r \sin (2 \pi k / K), R]^{T}$, for $k \in\langle K\rangle$. Note that all the column vectors have the same norm $\left\|\mathbf{a}_{k}\right\|=\sqrt{R^{2}+r^{2}}$. With $r=1, R=2$, the column vectors of $\mathbf{A}_{\circ}^{(K)}$ for $K=3,6,12$, and 24 , are shown in Table VI. The geometric representations for the corresponding polar zonohedra obtained using Algorithm 1 and Theorem 1 are shown in Fig. 15, where the edges are depicted as colored arrows that indicate the directions of the vectors $\mathbf{a}_{k}$ that span the facets and facilitate the identification of the edge and vertex associations indicated by Clauses 4 and 5 of Theorem 1. Specifically, for each sequence of magenta and cyan arrows, in that order and in the direction indicated by the arrows, the facet shared by the arrows is the facet with which the corresponding edges associate as per Clause 4. For each such sequence, the blue circle where the magenta arrow terminates and the cyan arrow begins identifies the vertex that associates with the same facet as per Clause 5. Note that for each facet the cross-product between the vector for the associated magenta edge and the vector for the associated cyan edge, in that order, consistently points into the gamut. 


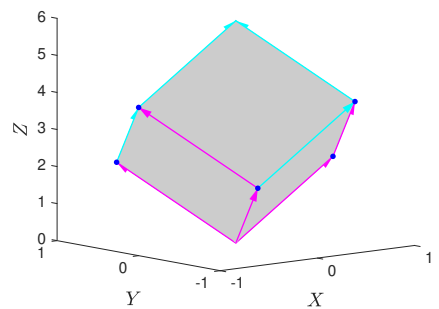

(a) $K=3$

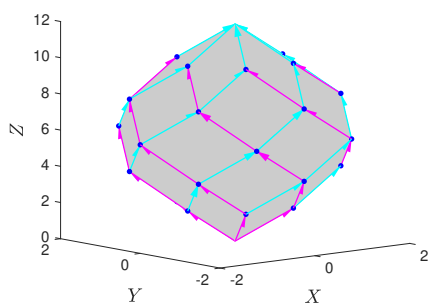

(c) $K=6$

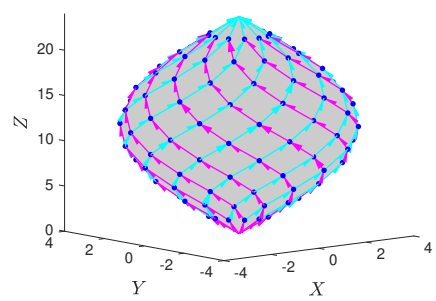

(e) $K=12$

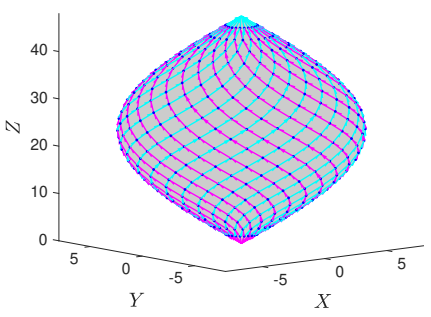

(g) $K=24$

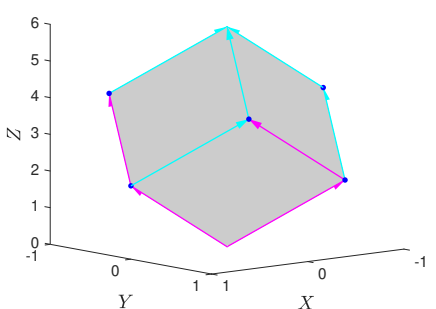

(b) $K=3$

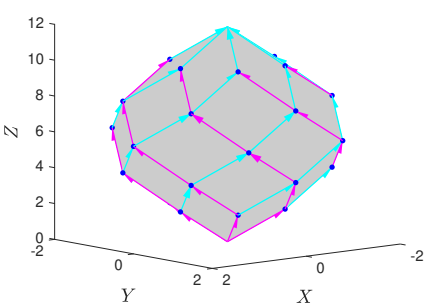

(d) $K=6$

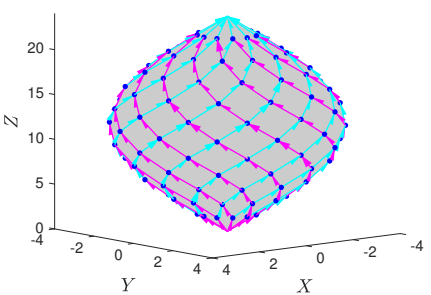

(f) $K=12$

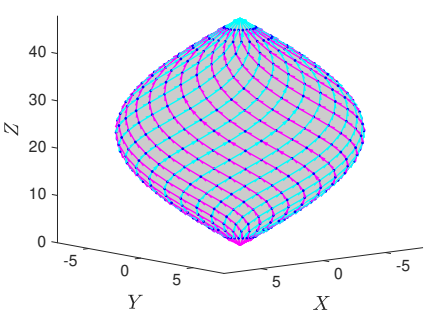

(h) $K=24$

Fig. 15. Polar zonohedra generated by the matrices $3 \times K$ matrices $\mathbf{A}_{\circ}^{(K)}$, with $r=1, R=2$, and $K=3,6,12,24$ (See description in text). The subfigures in each row illustrate two views of the corresponding polar zonohedron. For instance, (a) and (b) are views for the $K=3$ polar zonohedron. 


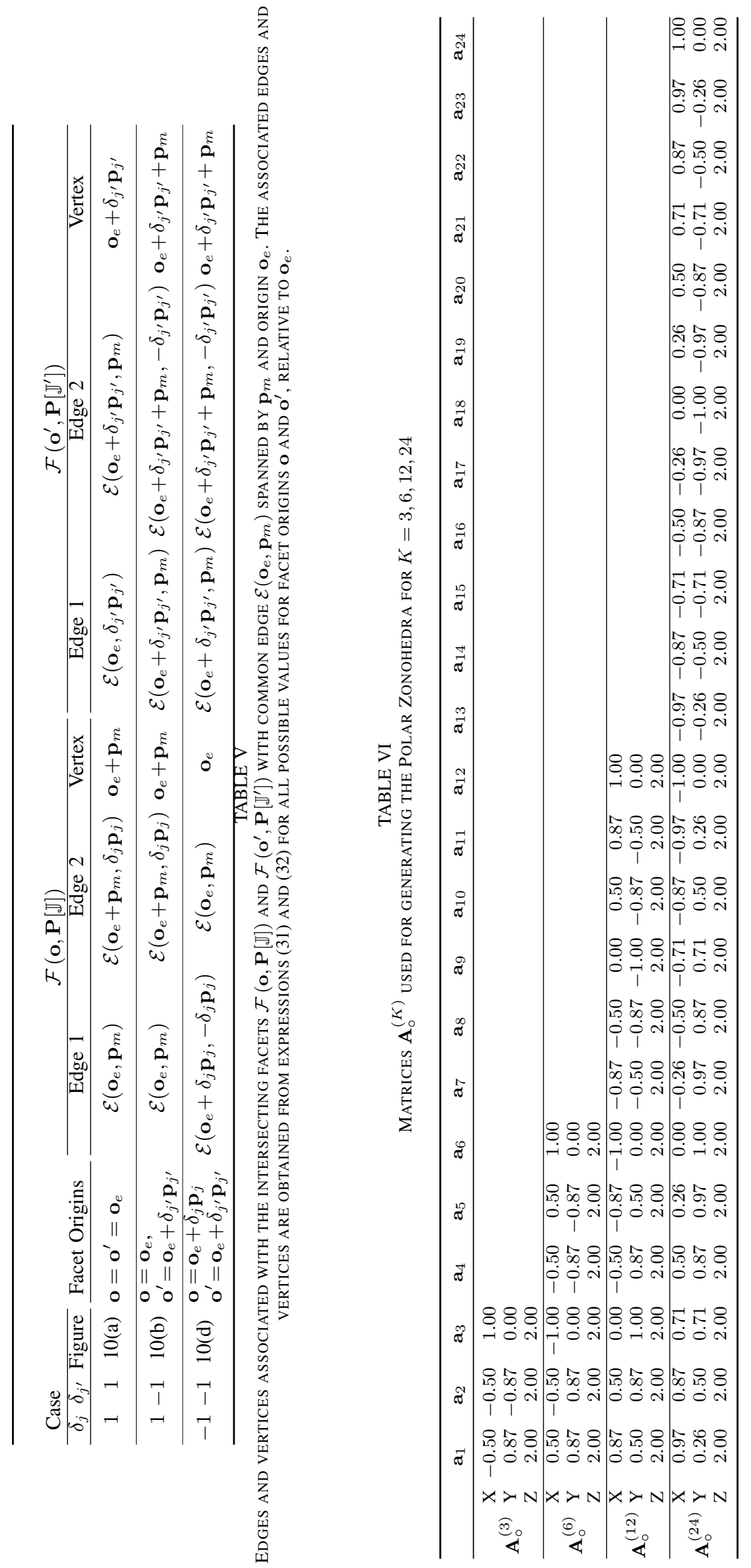




\section{REFERENCES}

[1] CIE, "Colorimetry," CIE Publication No. 15:2004, Central Bureau of the CIE, Vienna, 2004.

[2] H. J. Trussell, "Application of set theoretic models to color systems," Color Res. Appl., vol. 16, no. 1, pp. 31-41, Feb. 1991.

[3] G. Sharma and H. J. Trussell, "Digital color imaging," IEEE Trans. Image Proc., vol. 6, no. 7, pp. 901-932, Jul. 1997.

[4] M. J. Vrhel and H. J. Trussell, "Color device calibration: A mathematical formulation," IEEE Trans. Image Proc., vol. 8, no. 12, pp. 1796-1806, Dec. 1999

[5] R. Bala and G. Sharma, "System optimization in digital color imaging: Understanding and exploiting interactions," IEEE Sig. Proc. Mag., vol. 22, no. 1, pp. 55-63, Jan. 2005.

[6] G. Sharma, "LCDs versus CRTs: Color-calibration and gamut considerations," Proc. IEEE, vol. 90, no. 4, pp. 605-622, Apr. 2002.

[7] C. E. Rodríguez-Pardo, G. Sharma, J. Speigle, X. Feng, and I. Sezan, "Efficient computation of display gamut volumes in perceptual spaces," in Proc. IS\&T/SID Nineteenth Color and Imaging Conference: Color Science and Engineering Systems, Technologies, and Applications, San Jose, CA, 7-11 Nov. 2011, pp. 132-138.

[8] P. Centore, "A zonohedral approach to optimal colours," Color Res. Appl., vol. 38, no. 2, pp. 110-119, 2011.

[9] P. Centore and M. H. Brill, "Extensible multi-primary control sequences,” J. Soc. Inf. Display, vol. 20, no. 1, pp. 12-21, 2012.

[10] G. M. Ziegler, Lectures on polytopes, ser. Graduate Texts in Mathematics. New York, NY: Springer Science \& Business Media, 2007, vol. 152.

[11] P. Centore, "Non-metamerism of boundary colors in multi-primary displays," J. Soc. Inf. Display, vol. 20, no. 4, pp. 214-220, 2012.

[12] C. E. Rodríguez-Pardo and G. Sharma, "Geometry of multiprimary display colors II: Metameric control sets and gamut tilings," submitted for review.

[13] B. Grünbaum, Convex Polytopes, 2nd ed., ser. Graduate Texts in Mathematics. New York, NY: Springer-Verlag, 2003, vol. 221.

[14] MediaWiki, "Orientation," in Encylopedia of Mathematics. European Mathematical Society, 2019, accessed 26 July 2019. [Online]. Available: https://www.encyclopediaofmath.org/index.php/Orientation

[15] K. Jänich, Vector analysis. New York: Springer-Verlag, 2001.

[16] S. R. Lay, Convex sets and their applications. Malabar, FL: Krieger Publishing Company, 1992.

[17] E. J. Giorgianni, T. E. Madden, and K. E. Spaulding, "Color management for digital imaging systems," in Digital Color Imaging Handbook, G. Sharma, Ed. Boca Raton, FL: CRC Press, 2003, chapter 4.

[18] J. Morovič, Color gamut mapping. Chichester, West Sussex, England: John Wiley \& Sons, 2008, vol. 10

[19] C. Li, Z. Li, Z. Wang, Y. Xu, M. R. Luo, G. Cui, M. Melgosa, M. H. Brill, and M. Pointer, "Comprehensive color solutions: CAM16, CAT16, and CAM16-UCS," Color Res. Appl., vol. 42, no. 6, pp. 703-718, 2017.

[20] G. Sharma and C. E. Rodríguez-Pardo, "The dark side of CIELAB," in Proc. SPIE: Color Imaging XVII: Displaying, Hardcopy, Processing, and Applications, vol. 8292, Jan. 2012, pp. 8292-0D,1-10.

[21] K. Masaoka, Y. Kusakabe, T. Yamashita, Y. Nishida, T. Ikeda, and M. Sugawara, "Algorithm design for gamut mapping from UHDTV to HDTV," J. Display Technol., vol. 12, no. 7, pp. 760-769, July 2016.

[22] E. Sikudová, T. Pouli, A. Artusi, A. O. Akyüz, F. Banterle, Z. M Mazlumoglu, and E. Reinhard, "A gamut-mapping framework for coloraccurate reproduction of HDR images," IEEE Comp. Graphics and Applic., vol. 36, no. 4, pp. 78-90, July 2016.

[23] C. E. Rodríguez-Pardo, G. Sharma, X. Feng, J. Speigle, and I. Sezan, "Optimal gamut volume design for three primary and multiprimary display systems," in Proc. SPIE: Color Imaging XVII: Displaying, Hardcopy, Processing, and Applications, vol. 8292, Jan. 2012, pp. 8292$0 \mathrm{C}, 1-7$.

[24] L. Beke, Y. Kwak, P. Bodrogi, S. D. Lee, D.-S. Park, and C. Y. Kim, "Optimal color primaries for three- and multiprimary wide gamut displays," J. Electronic Imaging, vol. 17, no. 2, p. 023012, 2008.

[25] S. Wen, "Design of relative primary luminances for four-primary displays," Displays, vol. 26, no. 4-5, pp. 171 - 176, 2005

[26] K. Masaoka, Y. Nishida, M. Sugawara, and E. Nakasu, "Design of primaries for a wide-gamut television colorimetry," IEEE Trans. Broadcast., vol. 56, no. 4, pp. 452-457, Dec 2010.

[27] K. Masaoka, Y. Nishida, and M. Sugawara, "Designing display primaries with currently available light sources for UHDTV wide-gamut system colorimetry," Opt. Express, vol. 22, no. 16, pp. 19069-19077, 2014.

[28] C. Meyer, Matrix analysis and applied linear algebra. Philadephia, PA: SIAM, 2000
[29] "Recommendation ITU-R BT 709-6, Parameter values for the HDTV standards for production and international programme exchange," Jun. 2015. [Online]. Available: https://www.itu.int/rec/R-REC-BT.709/

[30] "Recommendation ITU-R BT. 2020-2. Parameter values for UHDTV systems for production and international programme exchange," Oct. 2015. [Online]. Available: https://www.itu.int/rec/R-REC-BT.2020/

[31] M. Pointer, "The gamut of real surface colours," Color Res. Appl., vol. 5, no. 3, pp. $145-155,1980$

[32] JIS, "Standard object colour spectra database for colour reproduction evaluation (SOCS)," Japanese Industrial Standard Technical Report (JISTR) X-0012, 1998.

[33] C. E. Rodríguez-Pardo and G. Sharma, "Color control functions for multiprimary displays I: Robustness analysis and optimization formulations," IEEE Trans. Image Proc., vol. 29, no. 1, pp. 1152-1163, 2020.

[34] T. Ajito, K. Ohsawa, T. Obi, M. Yamaguchi, and N. Ohyama, "Color conversion method for multiprimary display using matrix switching," Opt. Rev., vol. 8, no. 2, pp. 191-197, 2001.

[35] B. Chilton and H. Coxeter, "Polar zonohedra," Amer. Math. Monthly, pp. 946-951, 1963.

[36] H. Anton and R. G. Busby, Contemporary Linear Algebra. Hoboken, NJ: John Wiley and Sons, 2003.

[37] Proc. SPIE: Color Imaging XVII: Displaying, Hardcopy, Processing, and Applications, vol. 8292, Jan. 2012. 